\title{
Branes between Geometry and Gauge Theory
}

\author{
Dissertation \\ zur Erlangung des akademischen Grades \\ doctor rerum naturalium \\ (Dr. rer. nat.) \\ im Fach Physik \\ eingereicht an der \\ Mathematisch-Naturwissenschaftlichen Fakultät I \\ der Humboldt-Universität zu Berlin \\ von \\ Herrn Dipl.-Phys. André Miemiec \\ geboren am 22.09.1971 in Berlin
}

Präsident der Humboldt-Universität zu Berlin:

Prof. Dr. Dr. h.c. H. Meyer

Dekan der Mathematisch-Naturwissenschaftlichen Fakultät I:

Prof. Dr. J. P. Rabe

Gutachter:

1. Prof. Dr. D. Lüst

2. Prof. Dr. R. Schrader

3. Dr. sc. H. Dorn

eingereicht am:

22. März 2000

Tag der mündlichen Prüfung: $\quad$ 10. Juli 2000 


\begin{abstract}
The subject of this work is part of the theory of strings and in particular concerned with geometrical aspects of the realization of gauge theory within string theory. In particular we study the gauge theory of D3-branes at generalised singularities like orbifolds and the orbifolded conifold singularities. The introduction of the so called diamonds leads to a consistent picture. The action of mirror symmetry for this class of models is discussed.

In a second part the field theory of the M5-brane will be investigated on the basis of the superembedding approach. The main result here will be a rather explicit identification of the self dual three form field with the parameters of the complex structure of the embedding space.

The heuristic material collected from the previous chapters states that the information contained in supersymmetric 3-cycles can be partially extracted from supersymmetric 2-cycles. Thus we study the case of brane boxes, which can be obtained by sewing together usual $N=2$ setups of D4 and NS5-branes. These systems satisfy the condition of uniform bending and the lift to a supersymmetric 3-cycle can be performed explicitly.

In the last part a new superconformal $\mathrm{N}=1$ gauge theories will be constructed which arise as certain deformations of $\mathrm{N}=4 \mathrm{SYM}$ and have a dual description due to the AdS/CFT correspondence.
\end{abstract}

\title{
Keywords:
}

String theory, Branes, Gauge theory, AdS/CFT 


\section{Zusammenfassung}

Der Gegenstand dieser Arbeit ist Bestandteil der Stringtheorie und betrifft insbesondere die geometrischen Aspekte der Realisierung von Eichtheorien innerhalb der Stringtheorie. Wir studieren die Eichtheorie von D3-Branes an verallgemeinerten Singularitäten wie der Orbifold- oder Conifold-Singularität. Die Einführung der sogenannten Branediamanten führt auf ein konsistentes Bild. Für diese Klasse von Modellen wird die Wirkung der Mirrorsymmetrie diskutiert. Im zweiten Teil untersuchen wir die Feldtheorie der M5-Brane auf der Grundlage des Supereinbettungsformalismus. Das Hauptresultat dieses Kapitels ist eine explizite Identifikation der selbstdualen 3-Form mit den Parametern der komplexen Struktur des Einbettungsraumes.

Das heuristische Material, das in den vorangegangenen Kapiteln angesammelt wurde, weist darauf hin, daß die Informationen, die ein supersymmetrischer 3-Zykel enthält, zumindest teilweise auf supersymmetrische 2-Zykel zurückgeführt werden können. Daher studieren wir den Fall von Brane-Boxen, die durch Überlagerung von gewöhnlichen N=2 Systemen aus D4 und NS5-Branes gewonnen werden können. Diese Konfigurationen erfüllen die Bedingung des 'uniform bending' und der Lift auf einen supersymmetrischen 3-Zykel kann explizit ausgeführt werden.

Im letzten Teil konstruieren wir eine neue superkonforme $\mathrm{N}=1$ Eichtheorie, die aus einer Massendeformation der N=4 SYM entsteht und eine duale Supergravitationsbeschreibung durch die AdS/CFT-Korrespondenz besitzt.

\section{Schlagwörter:}

Stringtheorie, Branes, Eichtheorie, AdS/CFT 


\section{Contents}

$\begin{array}{ll}\text { Preface } & 1\end{array}$

1 Preliminaries $\quad 4$

1.1 M-Theory .......................... 4

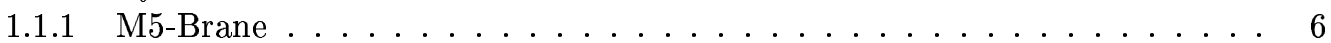

1.1.2 Dimensional Reduction to Type IIA SUGRA . . . . . . . . . . . . . 8

1.1.3 Dimensional Reduction of the M5-Brane . . . . . . . . . . . . 10

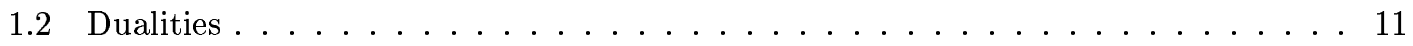

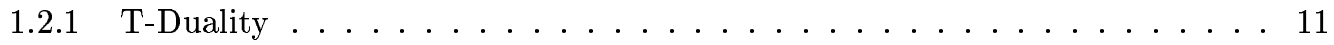

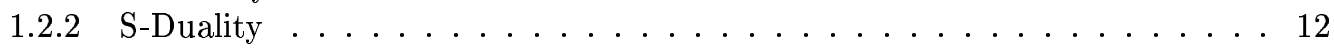

1.2.3 Intersecting BPS-Solutions . . . . . . . . . . . . . . 13

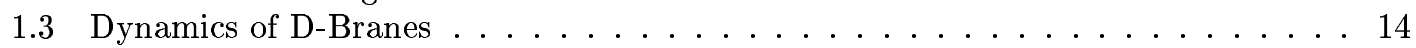

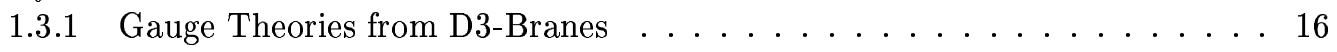

2 Branes at Singularities $\quad 21$

2.1 Toric Description of Singularities . . . . . . . . . . . . . . . . . . . . . . . . . . . .

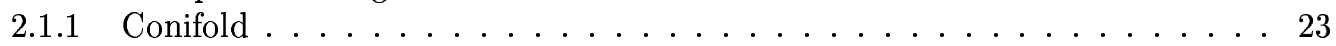

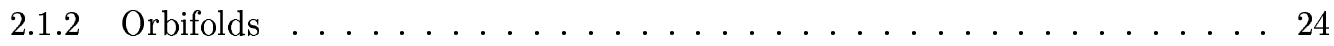

2.1.3 Hyperquotient Singularities . . . . . . . . . . . . . . . . 24

2.1.4 Generalised Conifold . . . . . . . . . . . . . . . . 25

2.2 Local Mirror Symmetry \& dual Geometries . . . . . . . . . . . . . . . . . . . . . . . . . . . . . . . 6

2.3 The Gauge Theories . . . . . . . . . . . . . . . . . . . . . . . . . . . . . . . . . . . . . . . . . . . .

2.4 The associated Brane Picture . . . . . . . . . . . . . . . . . 29

2.4.1 The Brane Box and its both T-dual Geometries . . . . . . . . . . . . . 31

2.4.2 Going to the Interval through a Conifold Transition . . . . . . . . . . . . 33

2.5 Probing the Mirror Geometries . . . . . . . . . . . . . . . . . 34

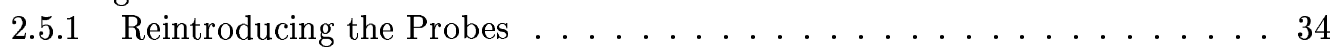

2.5.2 Generalised Conifold and Interval . . . . . . . . . . . . . . 35

2.5.3 D5-Branes on the Box, the modified Box Rules . . . . . . . . . . 35

2.6 Concluding Remarks . . . . . . . . . . . . . . . . . 40

3 BPS M5-Branes $\quad 41$

3.1 The $\mathbf{S U}(\mathbf{n})-\mathrm{SLAG}$ Calibrations . . . . . . . . . . . . . . . . . . . . . 42

3.2 A Generic Property of Lagrangian Submanifolds . . . . . . . . . . . . . . . 44 
3.3 Modified BPS Equations . . . . . . . . . . . . . . . . 46

3.3.1 Investigation of the Basic Example . . . . . . . . . . . . . 48

3.3 .2 The 3 -Cycle Case . . . . . . . . . . . . . . . . . . . 51

3.4 Concluding Remarks . . . . . . . . . . . . . . . . . . 54

$4 \mathrm{~N}=1$ Gauge Theories \& 3-Cycles

$4.1 \quad N=1$ Brane Boxes . . . . . . . . . . . . . . . . . . . . 59

4.2 T-Duality, M-Theory Embedding and the Emergence of the 3-Cycles . . . . . . . . 63

4.3 Brane Cubes and M-Theory . . . . . . . . . . . . . . . . . 65

4.4 Supersymmetric 3 -Cycles . . . . . . . . . . . . . . . . . . 65

4.5 Supersymmetric 3-Cycles for $N=1$ Brane Boxes $\ldots \ldots \ldots \ldots \ldots$. . . . . 67

4.5.1 Branes as Quaternionic Coordinates . . . . . . . . . . . . . 67

4.5.2 Uniform Bending - Sewing of $N=2$ Models . . . . . . . . . . . 69

4.6 Concluding Remarks . . . . . . . . . . . . . . . . . 72

5 Gauge Theory and Supergravity $\quad \mathbf{7 6}$

5.1 Construction of the New Conformal Theories . . . . . . . . . . . 78

5.2 The Flow from $N=4$ to $N=1$ Theories $\ldots \ldots \ldots \ldots$. . . . . . . . . 79

5.3 The dual Supergravity Description . . . . . . . . . . . . . . . . 81

$\begin{array}{lc}\text { A Definitions } & 86\end{array}$

$\begin{array}{ll}\text { B Toric Data of Singularities } & 92\end{array}$

C SLAG $d$-Cycles $\quad \mathbf{9 5}$

C.1 The $d$-Cycle Equations . . . . . . . . . . . . . . . . . . . . . . . . . . . . 95

C.2 An Algebraic Identity . . . . . . . . . . . . . . . . . . . 101

C.3 Graßmannians of 2-Planes in $\mathbb{R}^{4} \ldots \ldots \ldots \ldots 101$ 


\section{Preface}

Fede è sustanza di cose sperate e argomento de le non parventi; e questa pare a me sua quiditate.

Paradiso, Canto XXIV

This is a summary of the results obtained in my research toward the academic degree "doctor rerum naturalium". The subject of this work is part of the theory of strings and in particular concerned with geometrical aspects of the realization of gauge theory within string theory. The main tools for these investigations are solitonic solutions (branes) of the effective equations of motions, which describe the low energy behaviour of string theories. The effective theories we want to consider are the IIA and IIB supergravities and the 11 dimensional supergravity of [1], respectively. The latter is introduced since it helps to unify a lot of properties into a common picture. So by the Kaluza-Klein mechanism and T-duality the vast number of IIA and IIB-branes can be traced back to only two kinds of brane solutions of the 11 dimensional theory (M-theory), called M2 and M5-brane. In fact the study of quantum properties of the gauge theories living on IIA branes can be obtained from classical solutions of the M5-brane as shown in [2].

In chapter 1 we introduce the basic objects necessary for the description of gauge theories in stringy terms. Here we follow the approach of constructing as much as possible from 11 dimensional supergravity. A central place is assigned to the M5-brane solution, whose worldvolume theory is described. BPS-solutions of the M5-brane will be of dominant interest in chapters 3 and $4 . T$ as well as $S$-duality are examined, since they relate different brane setups to singularities. The worldvolume theories of Dirichlet branes (D-branes) are briefly discussed. For the gauge theory of $n$ parallel D3-branes at a orbifold singularity the orbifold projection is shown explicitly using the tools of [3].

In chapter 2 [4] we study the gauge theory of D3-branes at generalised singularities. For two classes of non compact Calabi-Yau threefolds the T-dual Neveu-Schwarz five brane (NS5-brane) configurations are investigated. It turns out that blowups (deformations) of orbifolded conifold singularities are $T$ dual to boxes (intervals) of NS5-branes, whereas blowups (deformations) of generalised conifold singularities are $T$ dual to intervals (boxes) of NS5-branes. The two singularities are in fact mirror pairs. The Kähler resp. complex structure parameters of the geometric singularities correspond to positions of the NS5-branes in the dual brane picture. Moreover a conifold transition for the mirror pairs via shrinking 2-cycles and growing up 3-cycles corresponds to the transition between the box and interval theory. These geometric singularities as well as the NS5-brane configurations serve as backgrounds, which are probed by a certain number 
of D-branes.

In order to establish the duality between conifold singularities and brane boxes we had to generalise the concept of brane boxes by also including brane diamonds. As a result we can formulate rules for deriving the matter content of the gauge theories living on boxes and diamonds generalising the rules of [5].

For certain geometries, blowups (deformations) allow both for a dual brane box as well as for a dual interval description. It follows that the corresponding gauge theory on the interval and on the brane box are mirror to each other. This observation is interesting with respect to the nonperturbative description of these kind of $N=1$ gauge theories: namely for every supersymmetric 2-cycle which describes the dynamics of the interval theory embedded in M-theory, there should exist a mirror supersymmetric 3-cycle for the brane box theory also embedded in M-theory.

In chapter 3 [6] we begin a slightly more formal investigation of the M5-brane. This was inspired by the arguments of chapter 2 which point out an equivalence in the description of gauge theories by supersymmetric 2-cycles and 3-cycles respectively. We will push forward this idea by collecting facts from concrete computations, which provide evidence in this respect. The basis of this investigation is the intrinsic formulation of the M5-brane worldvolume theory, developed in [7]. The main new result of this chapter will be the derivation of the differential equations ruling the structure of M5-branes in the presence of the self dual three form field $H$, living on the brane. In the case of the modified supersymmetric 2-cycle a rather explicit identification of the three form field $H$ with the parameters of complex structures on $\mathbb{R}^{4}$ is possible. For the case of supersymmetric 3-cycles, the closure of $H$ will become a deformed Laplace equation which should admit quite nontrivial solutions.

This results are closely connected to the results of [8]. There it was shown that the calibration bound can be cast into a form which estimates the energy of the M5-brane but the geometrical picture remained obscure.

In chapter 4 [9] we resume the discussion of brane boxes. The heuristic material collected from the previous chapters states that the information contained in 3-cycles can be partially extracted from 2-cycles. Thus we study the case of brane boxes, which can be obtained by sewing together usual $N=2$ setups of D4 and NS5-branes [10]. These systems satisfy the condition of uniform bending [11] and the lift to a supersymmetric 3 -cycle can be performed explicitly. The 3-cycles do have a rather special structure and can be constructed out of the 2-cycles describing the $N=2$ subsystems. We show that the so obtained 3 -cycle contains the information about the $\beta$-function by extracting the coefficient $b_{0}$ from the cycle. 
The discussion of D3-branes at singularities in chapter 2 is closely related to the by now famous AdS/CFT correspondence [12]. It provide us with a dual description of the $N=4 \mathrm{SYM}$ on the worldvolume of D3-branes in terms of IIB supergravity on $\operatorname{Ad}_{5} \times S^{5}$. To study extensions of this correspondence one is led to discuss the gauge theories of branes at orbifold singularities. For six-dimensional orbifold singularities $\mathcal{O}=\mathbb{R}^{6} / \Gamma$, with $\Gamma$ a discrete group, this was discussed in $[13,14]$ with $S^{5}$ replaced by $S^{5} / \Gamma$. The corresponding orbifold gauge theory can be calculated, again using the methods of [3]. The conifold singularities were later obtained in [15], where for the simplest conifold instead of $S^{5}$ there appears the homogeneous Einstein space $T^{1,1}$. Further types of conifold singularities were recently discussed in [16].

In chapter 5 [17] new superconformal $N=1$ gauge theories will be constructed which arise as certain deformations of $N=4 \mathrm{SYM}$ and have a dual description as supergravity on $A d S_{5} \times M^{5}$, with $M^{5}$ a five-dimensional Einstein space. There we will argue that after giving a mass to one of the chiral multiplets of $N=4 \mathrm{SYM}$, the effective theory of massless fields is dual to an $A d S_{5} \times M^{5}$ like solution found in [18]. There a description of the dual gauge theory was still missing.

Finally I want to express my sincere thanks to Dr. Andreas Karch and Prof. Dr. Dieter Lüst, whose kind support and collaboration made this work possible. Furthermore I want to thank Mina Aganagic for pleasant collaboration. Last but not least I would like to thank Volker Braun, Dr. Ilka Brunner, Boris Körs, Axel Krause, René Reinbacher, Dr. Douglas J. Smith as well as the members of the group "Quantum field theory" at Humboldt University for valuable discussions. 


\section{Chapter 1}

\section{Preliminaries}

\section{$1.1 \quad$ M-Theory}

As a starting point for the organisation of the material necessary in the upcoming sections we choose the $11 \mathrm{~d}$ supergravity (M-theory) of [1], which is given by the Lagrangian

$$
\begin{aligned}
\mathcal{L}= & \frac{1}{4} e R-\frac{1}{2} e \bar{\psi}_{\mu} \Gamma^{\mu \nu \rho} D_{\nu} \psi_{\rho}-\frac{1}{4 \cdot 48} e G_{\mu \nu \rho \sigma} G^{\mu \nu \rho \sigma} \\
& -\frac{1}{192} e\left(\bar{\psi}_{\mu} \Gamma^{\mu \nu \alpha \beta \gamma \delta} \psi_{\nu}+12 \bar{\psi}^{\alpha} \Gamma^{\beta \gamma} \psi^{\delta}\right) G_{\alpha \beta \gamma \delta} \\
& +\frac{1}{4 \cdot 144^{2}} \epsilon^{\alpha_{1} \ldots \alpha_{4} \beta_{1} \ldots \beta_{4} \mu \nu \rho} G_{\alpha_{1} \ldots \alpha_{4}} G_{\beta_{1} \ldots \beta_{4}} C_{\mu \nu \rho}+(4 \text {-Fermi terms) }
\end{aligned}
$$

with flat $11 \mathrm{~d}$ gamma matrices $\Gamma^{a}$ and vielbein $e^{a}{ }_{\mu}$ defined with respect to the flat $11 \mathrm{~d}$ metric $\eta_{a b}=(-1,1, \ldots, 1)$ and $\kappa_{(11)}=1$. The corresponding action is invariant with respect to $N=1$ supertransformations acting by

$$
\begin{aligned}
\delta_{Q} e_{\mu}{ }^{a} & =\bar{\epsilon} \Gamma^{a} \psi_{\mu} \\
\delta_{Q} C_{\mu \nu \rho} & =-3 \bar{\epsilon} \Gamma_{[\mu \nu} \psi_{\rho]} \\
\delta_{Q} \psi_{\mu} & =D_{\mu} \epsilon+\frac{1}{288}\left(\Gamma_{\mu}^{\alpha \beta \gamma \delta}{ }_{\mu}-8 \Gamma^{\beta \gamma \delta} \delta_{\mu}^{\alpha}\right) \epsilon G_{\alpha \beta \gamma \delta}+(3 \text {-Fermi terms })
\end{aligned}
$$

on the space of fields. Here $G_{\mu \nu \rho \sigma}=4 \partial_{[\mu} C_{\nu \rho \sigma]}$ is the field strength of the 3 -form potential $C_{\mu \nu \rho}$ and the covariant derivative is defined with respect to the spin connection $\omega_{\mu}{ }^{a b}$ :

$$
D_{\mu} \psi=\partial_{\mu} \psi+\frac{1}{4} \omega_{\mu}^{a b} \Gamma_{a b} .
$$


In practice one investigates pure bosonic solutions, i.e. setting the spinor fields to zero $\left(\psi_{\mu}=0\right)$. Under this assumption the equations of motion are given by

$$
\begin{aligned}
R_{\mu \nu} & =\frac{1}{12}\left(G_{\mu \alpha_{1} \ldots \alpha_{3}} G_{\nu}{ }^{\alpha_{1} \ldots \alpha_{3}}-\frac{1}{12} G^{2} g_{\mu \nu}\right) \\
0 & =d * G+\frac{1}{2} G \wedge G
\end{aligned}
$$

with $R_{\mu \nu}=R_{\mu \sigma \nu}^{\sigma}$.

Finding solutions to the above action is an extensively studied problem. Setting the 3 -form $C$ and the fermionic fields to zero one has just to solve the classical problem of integrating the Einstein equations. In particular the flat 11-dimensional Minkowski space is a solution, which will become important in section 1.1.1.

A deep insight into the structure of solutions is given through the SUSY-algebra. On behalf of arguments from the theory of representations [19] one is able to deduce the possible central terms which may appear on the left hand side of the anticommutator of two supercharges $Q_{\alpha}$ and $Q_{\beta}$ ending up with $\left(C=\Gamma^{0}\right)$

$$
\left\{Q_{\alpha}, Q_{\beta}\right\}=\left(\Gamma^{\mu} C\right)_{\alpha \beta} P_{\mu}+\frac{1}{2}\left(\Gamma^{\mu \nu} C\right)_{\alpha \beta} Z_{\mu \nu}+\frac{1}{5 !}\left(\Gamma^{\mu_{1} \ldots \mu_{5}} C\right)_{\alpha \beta} Z_{\mu_{1} \ldots \mu_{5}} .
$$

Thinking of the SUSY-algebra as realized on the space of fields by $\delta_{Q}$ one can take advantage of it for constructing solutions even in the case of vanishing spinor fields. Bosonic solutions of the equations of motion which form a representation of the SUSYalgebra at the same time must satisfy a further constraint (Killing equation),

$$
0 \stackrel{!}{=} \delta_{Q} \psi_{\mu}=\left[D_{\mu}+\frac{1}{288}\left(\Gamma_{\mu}^{\alpha \beta \gamma \delta}-8 \Gamma^{\beta \gamma \delta} \delta_{\mu}^{\alpha}\right) G_{\alpha \beta \gamma \delta}\right] \epsilon,
$$

which strongly restricts the set of solutions. Since configurations of fields of this type form short representations of the SUSY-algebra [20], they share a lot of special properties. The shortening of representations is a well known effect which goes hand in hand with the saturation of a bound estimating the energy through central charges. To be more precise for a static solution which has a rest frame the SUSY-algebra allows to express the energy density $\mathcal{E}$ of the solution in terms of the central charges appearing on the right hand side:

$$
\mathcal{E}=|Z|
$$

Solutions of this type are called extreme or BPS (Bogomolnyi-Prasad-Sommerfield). The BPS solutions which carry the central 2 or 5 -form charge are the M2-brane and M5-brane, respectively. The corresponding supergravity solutions are constructed in $[21,22]$.

The M2-brane couples electrically to the 3-form $C$ whereas the M5-brane is the dual, 
magnetically charged object. At this step the notions 'electrically charged' and 'magnetically charged' do not have a real meaning and refer only to the fact that the M2-brane couples directly to the form $C$ while the M5-brane couples to $\tilde{C}$, which is locally defined by $d \tilde{C}=* d C$ and remind of the situation familiar from electrodynamics.

The M5-brane solution has the form

$$
\begin{aligned}
d s^{2} & =H^{-1 / 3}\left(-d t^{2}+d x_{1}^{2}+\ldots+d x_{5}^{2}\right)+H^{2 / 3}\left(d x_{6}^{2}+\ldots+d x_{10}^{2}\right) \\
G_{\alpha_{1} \ldots \alpha_{4}} & =-c \epsilon_{\alpha_{1} \ldots \alpha_{5}} \partial_{\alpha_{5}} H\left(x_{6}, \ldots, x_{10}\right), \quad c= \pm 1, \quad \Delta H=0
\end{aligned}
$$

and it is convenient to consider the fundamental M5-brane solution as corresponding to the choice $H=1+\frac{a}{r^{3}}$.

An important characteristics of a solution is its tension. To be precise the tension $T_{p}$ of an asymptotically flat p-brane solution is the mass per unit volume defined as a generalised ADM-energy [23] of the p-brane solution in question. In practice this computation can be performed by splitting the spacelike part of the metric $g_{i j}=\delta_{i j}+h_{i j}$ into a flat background part $\delta_{i j}$ and a field $h_{i j}$. Then one computes in the flat background the asymptotically defined energy

$$
T_{p}=\int_{S^{4}}\left(\partial^{n} h_{m n}-\partial_{m} h^{i}{ }_{i}\right) \frac{x^{m}}{r} d \Omega_{S^{4}}=8 \pi^{2} a
$$

For a extreme solution it is equal to the charge $Q, T_{p}=|Q|$, with $Q$ the $G$-flux trough the $S^{4}$. From what was said before about the SUSY-algebra one can compute the condition a preserved supersymmetry must satisfy. Since the M5-brane is aligned along the (012345)-directions one obtains in a rest frame $P_{\mu}=(-\mathcal{E}, 0 \ldots 0)$ an eigenvalue equation,

$$
\left\{Q_{\alpha}, Q_{\beta}\right\} \epsilon=\mathcal{E}\left(1-\Gamma^{012345}\right) \epsilon \stackrel{!}{=} 0
$$

whose eigenvectors coincide with the preserved supersymmetries. The matrix $\Gamma=\Gamma^{012345}$ fulfils

$$
\Gamma^{2}=1, \quad \operatorname{tr} \Gamma=0
$$

Thus the M5-brane preserves a half of the 32 supersymmetries in 11 dimensions. This is a typical behaviour for all types of p-brane solutions.

\subsubsection{M5-Brane}

So far the M5-brane was introduced as a genuine SUGRA solution. From an alternative point of view one could consider a M5-brane as a probe which detects the gravitational field without taking any influence on it. But doubtless this is an idealisation, too. Since the M5-branes carry charge they act as sources for the 4-form field strength and their 
presence modify the gravitational field. An obvious question is for the dynamics of the branes induced by this back reaction. There are two complementary approaches dealing with this problem. The worldvolume action approach of [24] and the covariant field equation approach of [7]. It seems to us that for the purpose of application the latter approach is more suitable. Since it is essential for the arguments in the chapters 3 and 4 we want to explain the idea behind it, now. An excellent recent review is [25].

We will have to distinguish the components of a worldvolume form written in a spacetime frame (the indices are lower Latin letters from the middle of the alphabet $m, n, \ldots$ ) and in a tangent frame (indices are lower Latin letters from the top of the alphabet $a, b, \ldots$ ). In case of conflicting notation we will note explicitly the frame we are working with.

In static gauge the embedding of the M5-brane into flat 11d spacetime is realized by a map $f$, which describes the dependence of the transverse coordinates $X^{n^{\prime}}, n^{\prime}=1, \ldots, 5$ on the brane coordinates $q_{m}, m=0, \ldots, 5 . n^{\prime}$ labels only the transverse coordinates. Furthermore there lives a two-form field $B_{m n}$ on the six-dimensional worldvolume of the M5-brane with field strength $H=d B$. A nonlinear self duality constraint is realized on the field $H$, so that the anti self dual part can be computed from the self dual one:

$$
H_{a b c}=\frac{1}{Q}\left(h_{a b c}+2(k h)_{a b c}\right) .
$$

Here $k_{a}^{b}$ and $Q$ are defined by $k_{a}^{b}=h_{a c d} h^{b c d}$ and $Q=1-\frac{2}{3} t r k^{2}$. The 3-form $h_{a b c}$ is self dual in the usual sense. The equations of motion of the M5-brane are obtained from the superembedding approach [7]:

$$
\begin{aligned}
G^{m n} \nabla_{m} \nabla_{n} X^{a^{\prime}} & =0 \\
G^{m n} \nabla_{m} H_{n p q} & =0
\end{aligned}
$$

The effective metric is given by $G^{m n}=e_{c}{ }^{m} \eta^{c a} m_{a}{ }^{d} m_{d}{ }^{b} e_{b}{ }^{n}$, which exhibits in a complicated way the $H_{a b c}$ dependency through $m_{a}{ }^{b}=\delta_{a}{ }^{b}-2 h_{a c d} h^{b c d}$. If one sets the 3 -form $H_{a b c}$ to zero $G^{m n}$ becomes identical to the usual metric $g^{m n}$ and the equations of motion reduce themself to the harmonic embedding condition or said differently the M5-brane becomes minimal embedded into spacetime. This is a very geometrical description which will attract much more attention after discussing the lessons learned from the BPS properties of the M5-brane.

As it was further shown in [7], in the transformation law of the residual spacetime supersymmetry a projector $\tilde{\Gamma}$ appears,

$$
\delta \Theta=(1-\tilde{\Gamma}) \epsilon, \quad \tilde{\Gamma}=-\frac{1}{6 !} \epsilon^{a_{1} \ldots a_{6}} \tilde{\Gamma}_{a_{1} \ldots a_{6}}+\frac{1}{3} h^{a_{1} a_{2} a_{3}} \tilde{\Gamma}_{a_{1} a_{2} a_{3}}
$$


with $\tilde{\Gamma}_{a}$ six-dimensional tangent frame gamma matrices of the M5-brane worldvolume. From this formula one can find supersymmetry preserving solutions by requiring $\delta \Theta \equiv 0$. Since the M5-brane breaks the $S O(1,10)$ Lorentz invariance to a residual $S O(1,5) \times S O(5)$ subgroup, the formulas can be written in a well adapted form. In particular the $11 \mathrm{~d}$ gamma matrices $\Gamma_{a}$ can be constructed out of $\operatorname{Spin}(1,5)$ and $\operatorname{Spin}(5)$ gamma matrices by a general property of Clifford algebras. For the case at hand (appendix A) we obtain:

$$
\begin{aligned}
\mathcal{C} \ell\left(\mathbb{R}^{1,5} \oplus \mathbb{R}^{5}\right) & \cong \\
j(v \oplus w) & =v \otimes\left(\mathbb{R}^{1,5}\right) \otimes \mathcal{C} \ell\left(\mathbb{R}^{5}\right) \\
& v \otimes \mathbb{1}+\tilde{\Gamma}_{7} \otimes w .
\end{aligned}
$$

$\tilde{\Gamma}_{7}$ is the chirality operator of the algebra $\mathcal{C} \ell\left(\mathbb{R}^{1,5}\right)$. Then the explicit coordinate expression of the last formula for a flat 11d SUGRA background as derived in [26] reads:

$$
\begin{aligned}
& \hat{\delta} \Theta_{\beta}{ }^{j}=\frac{-1}{2 \operatorname{det} e} \epsilon^{\alpha i}\left\{\partial_{a} X^{c^{\prime}}\left(\gamma^{a}\right)_{\alpha \beta}\left(\gamma_{c^{\prime}}\right)_{i}{ }^{j}-\frac{1}{3 !} \partial_{a_{1}} X^{c_{1}^{\prime}} \partial_{a_{2}} X^{c_{2}^{\prime}} \partial_{a_{3}} X^{c_{3}^{\prime}}\left(\gamma^{a_{1} a_{2} a_{3}}\right)_{\alpha \beta}\left(\gamma_{c_{1}^{\prime} c_{2}^{\prime} c_{3}^{\prime}}\right)_{i}{ }^{j}\right. \\
& \left.+\frac{1}{5 !} \partial_{a_{1}} X^{c_{1}^{\prime}} \partial_{a_{2}} X^{c_{2}^{\prime}} \partial_{a_{3}} X^{c_{3}^{\prime}} \partial_{a_{4}} X^{c_{4}^{\prime}} \partial_{a_{5}} X^{c_{5}^{\prime}}\left(\gamma^{a_{1} a_{2} a_{3} a_{4} a_{5}}\right)_{\alpha \beta}\left(\gamma_{c_{1}^{\prime} c_{2}^{\prime} c_{3}^{\prime} c_{4}^{\prime} c_{5}^{\prime}}\right)_{i}{ }^{j}\right\} \\
& -\frac{1}{2} \epsilon^{\alpha i}\left\{-h^{m_{1} m_{2} m_{3}} \partial_{m_{2}} X^{c_{2}^{\prime}} \partial_{m_{3}} X^{c_{3}^{\prime}}\left(\gamma_{m_{1}}\right)_{\alpha \beta}\left(\gamma_{c_{2}^{\prime} c_{3}^{\prime}}\right)_{i}{ }^{j}-\frac{1}{3} h^{m_{1} m_{2} m_{3}}\left(\gamma_{m_{1} m_{2} m_{3}}\right)_{\alpha \beta} \delta_{i}{ }^{j}\right\} \text {. }
\end{aligned}
$$

Here $\gamma^{i}$ are the chiral $\operatorname{Spin}(1,5)$ tangent space gamma matrices on the M5-brane and $\gamma^{\prime i}$ the $\operatorname{Spin}(5)$ tangent space gamma matrices of the transverse space. The components $h_{m_{1} m_{2} m_{3}}$ of the self dual 3-form refer to the spacetime frame. Without further specialisations the only solution is given by a flat M5-brane preserving one half of spacetime supersymmetry. On the other hand if there are some nontrivial identities of products of gamma matrices applied to the spinor $\epsilon$ one obtains differential equations which determine more general supersymmetric M5-brane solutions. The amount of supersymmetry preserved by such a solution is determined by the number of relations imposed on the 11d gamma algebra.

\subsubsection{Dimensional Reduction to Type IIA SUGRA}

By dimensional reduction [27] of the 11d SUGRA ( eq. (1.1) ) down to 10 dimensions one obtains the Lagrangian of type IIA supergravity. As usual this process will be performed by compactifying the 11 th coordinate on a circle of Radius $R_{11}$. The Kaluza-Klein metric to end up in the 10-dimensional string frame reads

$$
g_{\mu \nu}^{(11)}=e^{-\frac{2}{3} \phi}\left(\begin{array}{cc}
g_{\mu \nu}^{s t}+e^{2 \phi} A_{\mu}^{(1)} A_{\nu}^{(1)} & e^{2 \phi} A_{\mu}^{(1)} \\
e^{2 \phi} A_{\nu}^{(1)} & e^{2 \phi}
\end{array}\right) .
$$

The $g_{1010}^{(11)}$ component describes the metric in the internal circle. Therefore the physical radius should be $R_{11}=e^{\frac{2}{3} \phi} R$ with $R$ the length in the identification $x_{10}=x_{10}+2 \pi R$. 
But $\phi$ is a dynamical field, the dilaton. To get a meaningful constant one inserts the expectation value $\phi_{0}$ of $\phi$. The inverse of $R$ sets the Planck mass $m_{p}=R^{-1}$. From these formulas one can express the physical radius in terms of the Planck mass and $e^{\phi_{0}}$, the latter of which is precisely the string coupling $g_{s}$, ending up with

$$
R_{11}=m_{p}^{-1} g_{s}^{2 / 3}
$$

For the case at hand the decomposition of the 11 dimensional fields generates the following spectrum in 10 dimensions,

$$
\begin{aligned}
g_{\mu \nu} & \longrightarrow g_{\mu \nu} \oplus A_{\mu}^{(1)} \oplus \phi, \\
\psi_{\mu} & \longrightarrow \psi_{\mu}^{ \pm} \oplus \lambda^{ \pm} \\
C_{\mu \nu \rho} & \longrightarrow A_{\mu \nu \rho}^{(3)} \oplus B_{\mu \nu}^{(2)},
\end{aligned}
$$

and using the basic Kaluza-Klein formula $R_{(11)}=R_{(10)}-\left\|F^{(2)}\right\|^{2} / 4$ valid for $\phi=0$ as well as the behaviour of the Ricci scalar with respect to rescaling of the metric $\tilde{g}_{\mu \nu}=e^{2 \sigma} g_{\mu \nu}$,

$$
\tilde{R}_{(\mathrm{d})}=e^{-2 \sigma}\left[R_{(\mathrm{d})}-2(d-1) \Delta \sigma-(d-1)(d-2) \partial_{\mu} \sigma \partial^{\mu} \sigma\right],
$$

one obtains the action of IIA SUGRA in ten dimensions:

$$
\begin{aligned}
S_{s t}^{I I A}= & \frac{1}{2 \kappa_{10}^{2}} \int d x^{10} \sqrt{-g}\left[e^{-2 \phi}\left(R+4 \nabla_{\mu} \phi \nabla^{\mu} \phi-\frac{1}{2 \cdot 3 !} H^{2}\right)-\frac{1}{4} F^{(2) 2}\right. \\
& \left.\quad \frac{1}{2 \cdot 4 !}\left(F^{(4)}+A^{(1)} \wedge H\right)^{2}\right]+\frac{1}{4 \kappa_{10}^{2}} \int F^{(4)} \wedge F^{(4)} \wedge B^{(2)} \\
+ & \text { further terms containing fermions. }
\end{aligned}
$$

The 10 dimensional Newtonian coupling constant is given by the 11 dimensional one $\left(\kappa_{11}=1\right)$ by $\kappa_{10}^{2}=\kappa_{11}^{2} /(\pi R)$ and if one anticipates a formula following from the first identification of tensions in Tab. 1.1 the radius of the compactification can be written as

$$
R_{11}=\frac{g_{s}}{m_{s}}
$$

Since the parameter $g_{s}$ controls the string perturbation theory it is justifiable to state that 11d SUGRA describes the strong coupling limit of type IIA string theory.

For practical computations the action should be written in the Einstein frame

$$
g_{\mu \nu}^{s t}=e^{\frac{\phi}{2}} g_{\mu \nu}^{E}
$$

Brane solutions do have only one of the gauge fields turned on. Then the generic form of the action is

$$
S_{E}^{I I A}=\frac{1}{2 \kappa_{10}^{2}} \int d x^{10} \sqrt{|g|}\left[R-\frac{1}{2} \partial_{\mu} \phi \partial^{\mu} \phi-\sum_{n} \frac{1}{2 n !} e^{a_{n} \phi} F_{n}^{2}\right]
$$

with $a_{n}=(5-n) / 2$ for Ramond-Ramond fields and $a=-1$ for the Neveu-Schwarz field. 


\subsubsection{Dimensional Reduction of the M5-Brane}

What happens with the solitonic objects of 11d SUGRA introduced at the beginning in the process of dimensional reduction? A good deal of information can be deduced from the reduction of the 11d-superalgebra, which decomposes into

$$
\begin{aligned}
\left\{Q_{\alpha}, Q_{\beta}\right\}= & \left(\Gamma^{\mu} C\right)_{\alpha \beta} P_{\mu}+\left(\Gamma^{10} C\right)_{\alpha \beta} P_{10}+\left(\Gamma^{\mu} \Gamma^{10} C\right)_{\alpha \beta} Z_{\mu 10}+\frac{1}{2}\left(\Gamma^{\mu \nu} C\right)_{\alpha \beta} Z_{\mu \nu} \\
& +\frac{1}{4 !}\left(\Gamma^{\mu \nu \rho \sigma} \Gamma^{10} C\right)_{\alpha \beta} Z_{\mu \nu \rho \sigma 10}+\frac{1}{5 !}\left(\Gamma^{\mu_{1} \ldots \mu_{5}} C\right)_{\alpha \beta} Z_{\mu_{1} \ldots \mu_{5}} .
\end{aligned}
$$

Obviously the number of solitonic objects which carry central charges increases considerably. Each term in the list can be identified from the point of view of 11d SUGRA [19]. What are the possible descendants of the solitonic M5-brane solution discussed before? Since the M5-brane is magnetically charged with respect to the 3 -form $C$ of the $11 \mathrm{~d}$ SUGRA, which in terms of IIA fields reads

$$
C=A^{(3)}+B^{(2)} \wedge d x^{11}
$$

it is very simple to deduce from the two diagrams below

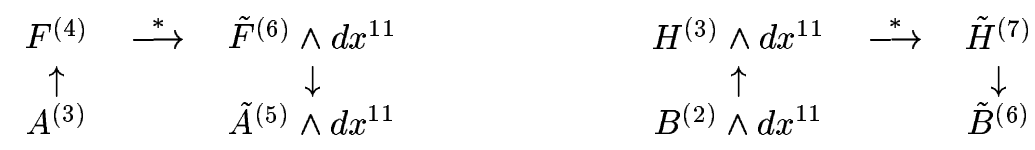

that after dimensional reduction the M5-brane wrapped around the circle gives raise to a (D)4-brane magnetically charged with respect to the Ramond-Ramond field $A^{(3)}$ whereas an unwrapped M5-brane produces an (NS)5-brane magnetically charged under the NS-NS 2-form $B^{(2)}$ (NS=Neveu-Schwarz). The reason for the different names of the p-forms appearing here is due to their origin from different sectors in string theory.

The explicit form of the D4-brane and NS5-brane solutions in the string frame can be computed most easily from the M5-brane solution ( eq. (1.9) ) simply by performing the dimensional reduction ( eq. (1.14) ) one time along a direction longitudinal and the other time transversal to the M5-brane combined with an appropriate Weyl rescaling to end up in the correct frame. For transversal reductions one has to handle a little subtlety concerning the dependencies of $H$ of the coordinate which will be reduced but then by straightforward application of the procedure described before one obtains in the string frame:

$$
\begin{aligned}
D 4: & d s^{2}=H^{-1 / 2}\left(-d t^{2}+d x_{1}^{2}+\ldots+d x_{4}^{2}\right)+H^{1 / 2}\left(d x_{5}^{2}+\ldots+d x_{9}^{2}\right) \\
& e^{2 \phi}=H^{-\frac{1}{2}}, \quad \Delta H\left(x_{5}, \ldots, x_{9}\right)=0, \quad H=1+\frac{a}{r^{3}}, \\
& F_{\alpha_{1} \alpha_{2} \alpha_{3} \alpha_{4}}=-c \epsilon_{\alpha_{1} \ldots \alpha_{5}} \partial_{\alpha_{5}} H\left(x_{5}, \ldots, x_{9}\right)
\end{aligned}
$$




$$
\begin{array}{ll}
N S 5: \quad & d s^{2}=\left(-d t^{2}+d x_{1}^{2}+\ldots+d x_{5}^{2}\right)+H\left(d x_{6}^{2}+\ldots+d x_{9}^{2}\right) \\
& e^{2 \phi}=H, \quad \Delta H\left(x_{6}, \ldots, x_{9}\right)=0, \quad H=1+\frac{a}{r^{2}} \\
& H_{\alpha_{1} \alpha_{2} \alpha_{3}}=-c \epsilon_{\alpha_{1} \ldots \alpha_{4}} \partial_{\alpha_{4}} H\left(x_{6}, \ldots, x_{9}\right)
\end{array}
$$

What's about the corresponding p-brane tensions ? From the definition of tension as mass per unit volume it must be proportional to the $p+1$ power of a fundamental mass scale of the theory (mass $\sim$ length $^{-1}$ ), i.e. $T_{p} \sim m^{p+1}$. The unique mass scale for M-branes is given by the $11 \mathrm{~d}$ Planck mass $m_{p}$. The factors of $R_{11}$ in the middle column of Tab. 1.1 are inserted by dimensional reasons.

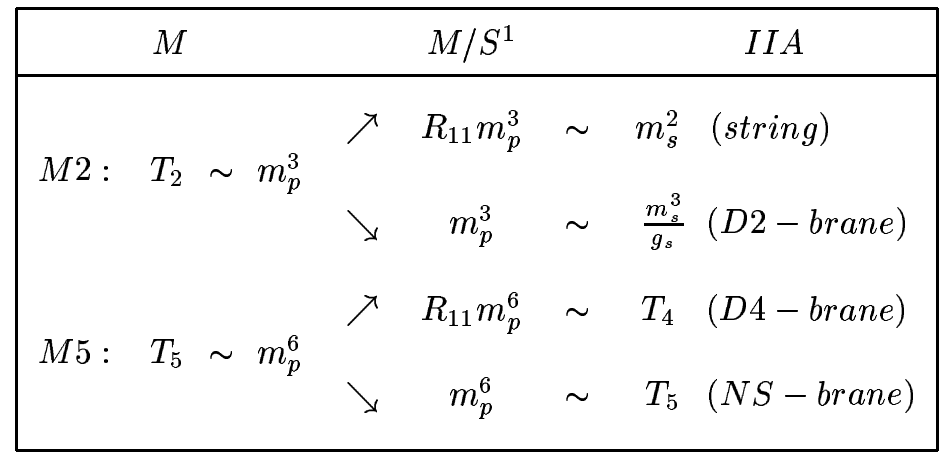

Tab. 1.1: Tensions of IIA Branes

If one identifies the tension of a wrapped M2-brane with the tension of a fundamental string and uses eq. (1.15) one finally obtains from Tab. 1.1:

$$
T_{4} \sim \frac{m_{s}^{5}}{g_{s}} \quad T_{5} \sim \frac{m_{s}^{6}}{g_{s}^{2}} .
$$

The tensions of the D4-brane and the NS5-brane exhibit a strong difference in their dependence on the string coupling $g_{s}$. This is a common feature to all D-branes. It is crucial for the discussion of the dynamical behaviour of these branes compared to each other. At weak string coupling the NS5-brane is much more heavier than a D-brane.

\subsection{Dualities}

\subsubsection{T-Duality}

Up to now we only considered $\mathrm{M}$ and IIA-branes. The third theory we announced in the preface was IIB supergravity [28]. Looking only for brane solutions the action can be written like the IIA action in eq. (1.21). The only difference is the degree of the Ramond-Ramond field strengths, which in IIA are even while in IIB odd. In addition the Ramond-Ramond five form is self dual. The NS5-brane of eq. (1.25) is also a solution 
of IIB theory. As shown in Fig. 1.1 below the IIA and IIB theories are connected trough a transformation, called T-duality $[29,30]$.

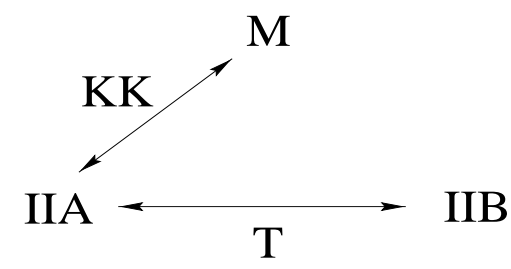

Fig. 1.1: Dualities of effective Theories

Here by T-duality we mean a transformation on the level of supergravity solutions admitting a killing vector (isometry). This parallels the usual T-duality in IIA and IIB string theories [31]. T-duality in string theory is a perturbative duality and one can regard the formulas below as valid on the lowest perturbative level, which are the supergravity theories. For a SUGRA solution it is simply defined by the map:

$$
\begin{aligned}
\tilde{G}_{0 i} & =G_{00}^{-1} B_{0 i} \quad \tilde{G}_{i j}=G_{i j}-G_{00}^{-1}\left(G_{i 0} G_{0 j}+B_{i 0} B_{0 j}\right) \quad \tilde{G}_{00}=G_{00}^{-1} \\
\tilde{B}_{0 i} & =G_{00}^{-1} G_{0 i} \quad \tilde{B}_{i j}=B_{i j}+G_{00}^{-1}\left(G_{i 0} B_{0 j}+B_{i 0} G_{0 j}\right) \\
\tilde{\phi} & =\phi-\frac{1}{2} \ln G_{00}
\end{aligned}
$$

Note that the 0 -direction denotes the direction of the isometry not time. The metric $G_{\mu \nu}$ is written in the string frame. From the D4-brane solution of type IIA one can generate a D5-brane solution of IIB by straightforward application of the above rules:

$$
\begin{aligned}
D 5: & d s^{2}=H^{-1 / 2}\left(-d t^{2}+d x_{1}^{2}+\ldots+d x_{5}^{2}\right)+H^{1 / 2}\left(d x_{6}^{2}+\ldots+d x_{9}^{2}\right) \\
& e^{2 \phi}=H^{-1}, \quad \Delta H\left(x_{6}, \ldots, x_{9}\right)=0, \quad H=1+\frac{a}{r^{2}}, \\
& F_{\alpha_{1} \alpha_{2} \alpha_{3}}=-c \epsilon_{\alpha_{1} \alpha_{2} \alpha_{3}} \partial_{\alpha_{3}} H\left(x_{6}, \ldots, x_{9}\right) .
\end{aligned}
$$

The structure of the Ramond-Ramond 3-form $F_{\alpha_{1} \alpha_{2} \alpha_{3}}$ is fixed by supersymmetry.

\subsubsection{S-Duality}

The full equation of motion of IIB supergravity are invariant under the combined transformations of the fields with respect to

$$
g=\left(\begin{array}{ll}
a & b \\
c & d
\end{array}\right) \in S L(2, \mathbb{R}) .
$$


This duality is called S-duality $[32,33]$ and the action on the fields looks like

$$
\begin{array}{cc}
g_{\mu \nu}^{E} \mapsto g_{\mu \nu}^{E} & F_{5} \mapsto F_{5} \\
\tau=A^{(0)}+i e^{-\phi} \mapsto \frac{a \tau+b}{c \tau+d} & \left(\begin{array}{c}
F_{3} \\
H_{3}
\end{array}\right) \mapsto\left(\begin{array}{ll}
a & b \\
c & d
\end{array}\right) \cdot\left(\begin{array}{c}
F_{3} \\
H_{3}
\end{array}\right)
\end{array}
$$

Here $A^{(0)}$ is the axion. Unlike T-duality which interchanges IIA and IIB solutions, S-duality stay inside the class of IIB solutions. An important consequence of S-duality is that it relates the NS5-brane to the D5-brane by the transformation $g=\left(\epsilon_{i j}\right)$, while inverting the string coupling at the same time.

\subsubsection{Intersecting BPS-Solutions}

All the fundamental brane solutions described so far can be used to build more complicated metrics by what is known as intersecting branes. Furthermore exploiting the two duality transformations discussed before on such configurations one can generate many more interesting solutions, which sometimes could be reinterpreted in terms of branes at singularities.

The basic example is already known for some time [34, 35]. There it was argued that the geometric orbifold and conifold singularities are T-dual to a certain number of NS5-branes. This T-duality will be used to transform D3-branes probing the singularity (cf. section 1.3.1) into a pure brane configuration of intersecting NS5-branes and D-branes of the Hanany-Witten type [5,36]. It is this fact that we will systematically explore in chapter 2. For convenience we will review the basic example of D4-branes between $k$ NS5-branes which is dual to D3-branes at the $\mathbb{Z}_{k}$-singularity [37]. It proves useful to start from the configuration in IIB below

\begin{tabular}{|l|lllllll|}
\hline D5 & 0 & 1 & 2 & 3 & 4 & 5 & \\
D3 & 0 & 1 & 2 & & & & 6 \\
\hline
\end{tabular}

Tab. 1.2: Intersecting configuration

for which the intersecting brane solution can be written down. There is a general rule to obtain the metric of a system of $N$ intersecting Dp-branes not necessarily of the same dimension $p$. Determine the number $m_{i}$ of Dp-branes which have $n_{i}$ common directions, with $n_{i}$ a decreasing sequence. The metric is given by,

$$
d s^{2}=\left(H_{1} \ldots H_{N}\right)^{1 / 2}\left[\left(H_{1} \ldots H_{m_{1}}\right)^{-1} d s_{01 \ldots n_{1}}^{2}+\left(H_{1} \ldots H_{m_{2}}\right)^{-1} d s_{n_{1}+1 \ldots n_{1}+n_{2}}^{2}+\ldots+d s_{\perp}^{2}\right],
$$

where $d s_{\perp}^{2}$ covers all the coordinates, which do not have a common directions with the branes involved. 
Then for the configuration of Tab. 1.2 one can determine the metric and the behaviour under a whole sequence of dualities:

$$
\begin{gathered}
d s^{2}=\left(H_{5} H_{3}\right)^{-1 / 2} d s_{012}^{2}+\left(H_{3} / H_{5}\right)^{1 / 2} d s_{345}^{2}+\left(H_{5} / H_{3}\right)^{1 / 2} d s_{6}^{2}+\left(H_{3} H_{5}\right)^{1 / 2} d s_{789}^{2} \\
\downarrow \text { S-duality } \\
\text { D3-brane between NS5-branes } \\
\downarrow T_{3} \text {-duality } \\
\text { D4-brane between NS5-branes } \\
\downarrow T_{6} \text {-duality } \\
d s^{2}=H_{3}^{-1 / 2} d s_{0123}^{2}+H_{3}^{1 / 2}\left[d s_{45}^{2}+H_{5}^{-1}\left(d x_{6}+\vec{\omega} \cdot \overrightarrow{d s} s_{789}\right)^{2}+H_{5} d s_{789}^{2}\right]
\end{gathered}
$$

Here $\operatorname{rot} \vec{\omega}=\operatorname{grad} H_{5}$ with $H_{5}$ the multi centre harmonic function

$$
H_{5}=\epsilon+\sum_{i=1}^{k} \frac{1}{\left|\vec{x}-\vec{x}_{i}\right|} .
$$

The solution has the form of a D3-brane solution, whose transversal space contains a Taub-NUT space in the 6789-directions. Near the brane one can neglect $\epsilon$ and the TaubNUT metric becomes the metric of the resolution of an $A_{k-1}$-singularity. The singular space can be obtained back by putting all NS5-branes at the same position $\vec{x}_{0}$.

In the same reference [37] it was shown, that the skeleton of $k$ NS5-branes in 012345 and $l$ NS5'-branes in 012367 are dual to a geometric singularity of type $\mathbb{C}^{3} / \mathbb{Z}_{k} \times \mathbb{Z}_{l}$ (this becomes important for the brane boxes of chapter 2). In [38] it was shown that the SUGRA solution of D4-branes between orthogonal NS5-branes can be mapped into a SUGRA solution of D3-branes at the conifold singularity ( this will be useful for the interval theory of chapter 2). The steps to establish this correspondence are very similar to that of the example described above. Further examples of dualities between geometries and brane configurations including orbifolded conifolds and generalised conifolds can be found in the literature $[39,16,40]$.

\subsection{Dynamics of D-Branes}

After having discussed the classical brane solutions to some extend we want briefly mention the origin of the field theory living on D-branes. This field theory is the essential ingredient for the realization of gauge theories inside string theory. As one can see from the explicit solutions, e.g. eq. (1.24), D-branes are charged with respect to the Ramond- 
Ramond fields. The same observation was made for spacetime defects on which open strings can end [41], which therefore becomes identified. The massless excitations of open strings, which couple to the Dp-brane, describe the low energy dynamics of the

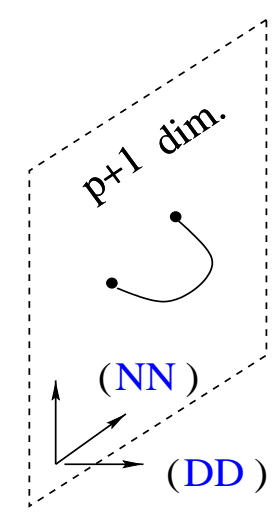

Fig. 1.2: Open string

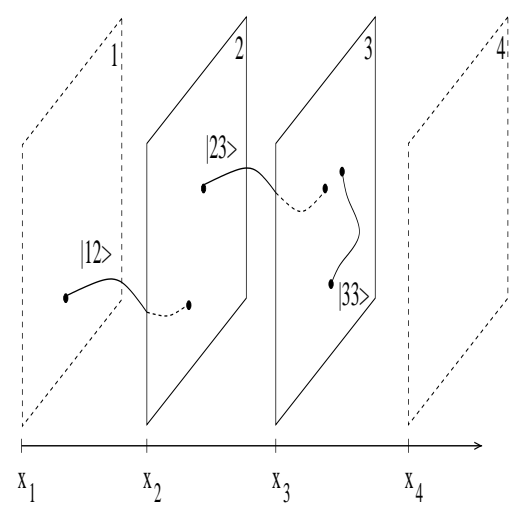

Fig. 1.3: $n$ D-branes D-brane. The open string wave function satisfy the equation $\left(\partial_{t}^{2}-\partial_{x}^{2}\right) X^{\mu}=0$. The endpoints are restricted to lie on the hypersurface depicted in Fig. 1.2. This can be implemented by imposing Dirichlet or Neumann boundary conditions on the two endpoints as shown in Fig. 1.2. For each direction the two endpoints fulfil the same boundary condition (DD or NN). The solution of the wave equation can be written as a Fourier expansion, with free coefficients $a_{ \pm l}^{\mu}$. The quantised system does have excited states of mass (energy)

$$
m^{2} \sim\left(\sum_{l=1}^{\infty} l \cdot N_{l}-1\right)
$$

with $N_{l}$ the number of particles excited in the l-th mode and $a_{l}^{\mu+}:=a_{-l}^{\mu}$ the creation operators. The massless states are the states with only one creation operator acting on the vacuum:

$$
\begin{array}{lll}
a_{1}^{\mu+} \mid k, i i> & \mu=0 \ldots p & \text { long. dir. } \\
a_{1}^{n+} \mid k, i i> & n=p+1 \ldots 9 & \text { transv. dir. }
\end{array}
$$

Since the first set of states are due to NN-directions they do not transfer a momentum to the brane. The states are confined to the brane and produce the degrees of freedom of a U(1)-gauge field $A_{\mu}$. The second set of states do have a momentum transfer and can be interpreted as scalar fields parametrising the position of the D-brane in the transverse directions. A slight generalisation is possible if one considers $n$ parallel branes as in

Fig. 1.3. There are open strings stretching from one brane to the other. Their mass is proportional to the distance:

$$
m \sim\left|x_{i}-x_{j}\right| .
$$

If two or more D-branes are on top of each other there are additional massless gauge fields, which lead to enhanced gauge symmetry. For $n$ coincident D-branes the gauge 
group becomes enhanced from $U(1)^{n} \rightarrow U(n)$.

This intuitive rules can be made more precise by the usual $\sigma$-model approach to string theory. If one considers the $\sigma$-model action below with boundary conditions as in Fig. 1.2,

$$
S_{\sigma}=-\frac{T_{2}}{2} \int d^{2} \xi\left(\sqrt{h} h^{\alpha \beta} \partial_{\alpha} X^{\mu} \partial_{\beta} X^{\nu} G_{\mu \nu}+\epsilon^{\alpha \beta} \partial_{\alpha} X^{\mu} \partial_{\beta} X^{\nu} B_{\mu \nu}-2 \pi \alpha^{\prime} \sqrt{h} R \Phi\right),
$$

one can derived the consistency conditions of the system in Fig. 1.2, required for conformal invariance. As was shown in $[42,43]$ these consistency conditions imply equations of motion of the Born-Infeld action of nonlinear electrodynamics

$$
S_{p}=-T_{p} \int d^{p+1} \xi e^{-\phi} \sqrt{\operatorname{det}\left(g_{\mu \nu}+B_{\mu \nu}+2 \pi F_{\mu \nu}\right)},
$$

which therefore is taken as the effective low energy description of the D-brane. In the non abelian situation a slight simplification is possible, if one considers flat spacetime and only weak nonlinearities. Then the effective action of a Dp-brane can be well approximated by the dimensional reduction of 10-dimensional, $\mathrm{N}=1$, supersymmetric U(n)-Yang-Mills theory (SYM) [44].

\subsubsection{Gauge Theories from D3-Branes}

A primary role in applications is played by the D3-brane. Here the dimension of the worldvolume is four, which matches with the number of dimensions visible to us. The IIB-SUGRA solution is simply given by [45]

$$
\begin{aligned}
D 3: & d s^{2}=H^{-1 / 2}\left(-d t^{2}+d x_{1}^{2}+\ldots d x_{3}^{2}\right)+H^{1 / 2}\left(d x_{4}^{2}+\ldots+d x_{9}^{2}\right) \\
& e^{\phi}=g_{s}, \quad \Delta H\left(x_{4}, \ldots, x_{9}\right)=0, \quad H=1+\frac{a}{r^{4}}, \\
& F_{0123 r}=\frac{\partial H^{-1}}{\partial r} \text { and } F_{\theta_{1} \ldots \theta_{5}}=\sqrt{-g} F^{0123 r} .
\end{aligned}
$$

The field theory of $n$ parallel D3-branes is $N=4$ supersymmetric $U(n)$ gauge theory with the action below:

$$
\begin{aligned}
\mathcal{L}_{N=4}= & \int d^{2} \theta d^{2} \bar{\theta}\left[\sum_{i=1}^{3} \bar{\Phi}_{i} e^{2 g V} \Phi_{i}\right]+\frac{1}{8 \pi} \Im \mathfrak{m}\left[\int d^{2} \theta \tau W^{\alpha} W_{\alpha}\right] \\
+ & g \frac{\sqrt{2}}{3 !}\left[\int d^{2} \theta \epsilon^{i j k} \Phi_{i}\left[\Phi_{j}, \Phi_{k}\right]+\text { h.c. }\right]
\end{aligned}
$$

In $N=4$ there is only the vector multiplet from which one can build the action. In terms of $N=1$ superfields it contains an $N=1$ vector multiplet and three chiral multiplets in the adjoint representation of the gauge group. The complex scalars of the chiral 
multiplets can be seen as the position coordinates of the D3-branes in the transverse directions. Furthermore the $R$-symmetry can be connected to the group of isometries of the transverse space.

To obtain gauge theories with lower amount of supersymmetry one uses a special orbifolding technique $[3,46]$. One considers the action of a discrete group $\Gamma$ on the transverse space, which defines the orbifold $\mathcal{O}=\mathbb{C}^{3} / \Gamma$. Generically this will break all of the supersymmetries by breaking the whole $R$-symmetry. If one chooses a proper embedding of $\Gamma$ into $S U(4)$ one could preserve a residual $R$-symmetry of lower supersymmetry. The corresponding choices are listed in Tab. 1.3:

\begin{tabular}{|ccc|}
\hline$\Gamma \subset$ & residual $R$-symmetry & $N$ \\
\hline 1 & $S U(4)$ & 4 \\
$S U(2)$ & $S U(2) \times U(1)$ & 2 \\
$S U(3)$ & $U(1)$ & 1 \\
$S U(4)$ & 1 & 0 \\
\hline
\end{tabular}

Tab. 1.3 : Broken $R$-Symmetries

As can be seen from the above list to preserve a certain fraction of SUSY the orbifold group $\Gamma$ must be embedded into an $S U$ subgroup of $S U(4)$. One obtains $N=1$ supersymmetric theories if one takes the generators of $\Gamma$ inside $S U(3)$. An explicit choice is given in appendix $\mathrm{B}$, where the embedding into $S U(3)$ is manifest. This will lead to an $\mathbb{Z}_{k} \times \mathbb{Z}_{l}$ orbifold $\mathcal{O}_{k l}$. From Tab. B.1 one can read off the action of $\Gamma$ on the coordinates. The orbifold action on the branes can be visualised by taking $k \cdot l$ copies of the original brane configuration. Then the group acts as a shift operation on the set of copies. If one starts with $n$ branes one may label each brane in the set of copies by a Chan-Paton index $i=1 \ldots k \cdot l \cdot n$. The action of the generator $g$ of $\mathbb{Z}_{k}$ on the set of all copies can be represented by the matrix

$$
\gamma(g)=\operatorname{diag}\left(\mathbb{1}_{l \cdot n}, g \mathbb{1}_{l \cdot n}, \ldots, g^{k-1} \mathbb{1}_{l \cdot n}\right),
$$

while the generator $h$ of $\mathbb{Z}_{l}$ acts like

$$
\gamma(h)=\operatorname{diag}\left(\mathbf{1}_{n}, h \mathbf{1}_{n}, \ldots, h^{l-1} \mathbb{1}_{n}, \ldots ., \mathbb{1}_{n}, h \mathbf{1}_{n}, \ldots, h^{l-1} \mathbf{1}_{n}\right)
$$

$\mathbb{1}_{m}$ is the unit matrix in $m$ dimensions. On fields carrying Chan-Paton labels, $\Gamma$ acts like

$$
A_{i j} \longrightarrow \gamma(a)_{i m} A_{m n} \gamma(a)_{n j}^{-1}
$$

The gauge theory at the orbifold point contains all the fields of the covering space invariant under the group $\Gamma$. The invariant gauge fields must commute with the $\gamma(g)$ 
and $\gamma(h)$ action. So only the entries of the right matrix survive:

$$
\left(\begin{array}{ccc}
A_{1,1} & \ldots & \cdot \\
\vdots & \ddots & \vdots \\
\cdot & \ldots & A_{k l n, k l n}
\end{array}\right) \longrightarrow\left(\begin{array}{ccc}
A_{n \times n} & 0 & 0 \\
0 & \ddots & 0 \\
0 & 0 & A_{n \times n}
\end{array}\right)
$$

This leads to the gauge group ${ }^{* 1} S U(n)^{k l}$. On the chiral matter fields $\Phi_{i j}^{\mu}$ in the adjoint representation there is a similar effect. But now one has to take the additional contribution from the action of $\Gamma$ on the vector index into account. The concrete computation is a little bit lengthy but we will include it for being self contained. To keep the formulas short we will suppress the vector index sometimes. To prepare the chain of arguments we would like to decompose the matrix containing all the chiral matter fields into blocks on which the Chan-Paton matrices act.

$$
\Phi=\left(\begin{array}{ccccc}
\tilde{X}_{1,1^{\prime}} & \ldots & \ldots & \ldots & \tilde{X}_{1, k^{\prime}} \\
\vdots & \ddots & & & \vdots \\
\vdots & & \tilde{X}_{i, i^{\prime}} & & \vdots \\
\vdots & & & \ddots & \vdots \\
\tilde{X}_{k, 1^{\prime}} & \ldots & \ldots & \ldots & \tilde{X}_{k, k^{\prime}}
\end{array}\right) \quad \tilde{X}_{i, i^{\prime}}=\left(\begin{array}{ccccc}
\bar{X}_{1,1^{\prime}} & \ldots & \ldots & \ldots & \bar{X}_{1, l^{\prime}} \\
\vdots & \ddots & & & \vdots \\
\vdots & & \bar{X}_{j, j^{\prime}} & & \vdots \\
\vdots & & & \ddots & \vdots \\
\bar{X}_{l, 1^{\prime}} & \ldots & \ldots & \ldots & \bar{X}_{l, l^{\prime}}
\end{array}\right)
$$

The crucial point to note is that $\gamma(g)$ acts only on the blocks $\tilde{X}$ but not inside $\tilde{X}$. Similar $\gamma(h)$ acts only inside $\tilde{X}$ (on the $\bar{X}$ ) but not on the blocks $\tilde{X}$. Therefore one can compute the invariant state by iterating the two orbifold projections. First look at the action of $h$ on $\tilde{X}$. One obtains:

$$
\gamma(h) \tilde{X} \gamma(h)^{-1}=\left(\begin{array}{ccccc}
\bar{X}_{1,1^{\prime}} & h^{-1} \bar{X}_{1,2^{\prime}} & \ldots & \ldots & h^{-(l-1)} \bar{X}_{1, l^{\prime}} \\
h \bar{X}_{2,1^{\prime}} & \ddots & & & \vdots \\
\vdots & & \bar{X}_{j, j^{\prime}} & & \vdots \\
\vdots & & & \ddots & \vdots \\
h^{l-1} \bar{X}_{l, 1^{\prime}} & \ldots & \ldots & \ldots & \bar{X}_{l, l^{\prime}}
\end{array}\right) .
$$

This has to be combined with the action of $\Gamma$ on the vector index. From Tab. B.1 one can read off that $\mathbb{Z}_{l}$ acts on the coordinates $Z^{\mu}$ of $\mathbb{C}^{3}$ like

$$
Z^{\mu} \rightarrow h^{a_{\mu}} Z^{\mu}:\left(a_{1}, a_{2}, a_{3}\right)=(-1,1,0)
$$

\footnotetext{
${ }^{* 1}$ One might wonder about the actual gauge group we are dealing with, which is not $U(n)^{k l}$ as one might expect. In the standard brane setup the $U(1)$ factors are frozen out by quantum effects for $d \geq 4$ dimensions. The reason is that the $U(1)$ corresponds to the centre of mass motion of the D-branes in the brane picture. Since for $d \geq 4$ there is a logarithmic (or worser) bending of branes this motion would change the asymptotic behaviour of the branes and is frozen out [2].
} 
which multiplies $\bar{X}^{\mu}$ by a certain power of $h$. As a result the invariant fields are indicated below

$$
\tilde{X}^{1}=\left(\begin{array}{cccc}
0 & & & \bar{X} \\
\bar{X} & \ddots & & \\
& \ddots & \ddots & \\
& & \bar{X} & 0
\end{array}\right) \quad \tilde{X}^{2}=\left(\begin{array}{cccc}
0 & \bar{X} & & \\
& \ddots & \ddots & \\
& & \ddots & \bar{X} \\
\bar{X} & & & 0
\end{array}\right) \quad \tilde{X}^{3}=\left(\begin{array}{cccc}
\bar{X} & & & \\
& \ddots & & \\
& & \ddots & \\
& & \bar{X}
\end{array}\right)
$$

The same arguments apply to the determination of invariant states for the $\gamma(g)$-action. The only difference is that we change the scope of resolution and work on the $\tilde{X}$-matrices, now. From the computation before we actually know how $\gamma(g)$ acts. The only thing which differs is the action of the group $\mathbb{Z}_{k}$ on the coordinates $Z^{\mu}$ :

$$
Z^{\mu} \rightarrow g^{a_{\mu}} Z^{\mu}:\left(a_{1}, a_{2}, a_{3}\right)=(-1,0,1) .
$$

Now we can simply put all pieces together, obtaining:

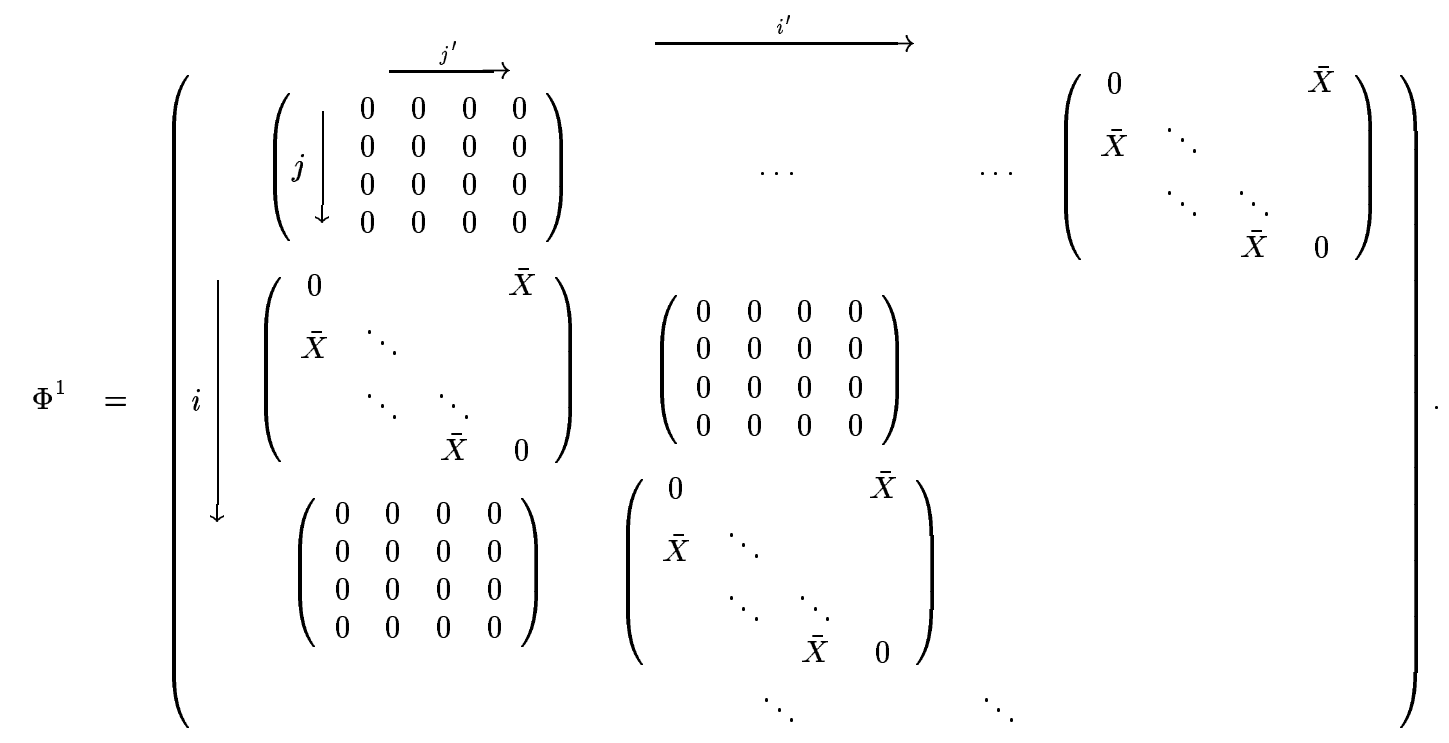

It is simple to read off the surviving fields. The first class of fields stems from the chiral $\Phi^{1}$ and transforms in the representation $(\square, \bar{\square})$ of

$$
S U\left(n_{i, j}\right) \times S U\left(n_{i-1, j-1}\right) .
$$

For obvious reasons concerning the index structure of the gauge groups involved they are called diagonal fields $D_{i j}$. The same steps can be repeated for the other two classes. Omitting the details the other two chiral fields descends to the 'horizontal' chiral bifundamentals $H_{i j}$ transforming in $(\square, \bar{\square})$ of $S U\left(n_{i, j}\right) \times S U\left(n_{i+1, j}\right)$ and the 'vertical' chiral bifundamentals $V_{i j}$ in the representations $(\square, \bar{\square})$ of $S U\left(n_{i, j}\right) \times S U\left(n_{i, j+1}\right)$. 
The superpotential consists of the terms surviving the projection and is of the form:

$$
W=\sum_{i j} H_{i, j} V_{i+1, j} D_{i+1, j+1}-\sum_{i j} V_{i, j} H_{i, j+1} D_{i+1, j+1} .
$$

A more striking method to derive the above results, which is in fact applicable to a wider class of models, was described in [14]. 


\section{Chapter 2}

\section{Branes at Singularities}

In this chapter we want to study the gauge theories living on D-branes, which probe the singular limits of typical compactification manifolds, the famous Calabi-Yau spaces.

We start to consider $n$ D3-branes probing a Calabi-Yau manifold $\mathcal{M}$. At a smooth point of $\mathcal{M}$ the tangent space is $\mathbb{R}^{6}$ and the D3-brane will have $N=4$ supersymmetry on the world volume. As explained in section 1.3.1 more interesting models arise, if one considers the action of a discrete group in the transversal directions or said differently if one considers singular Calabi-Yau spaces. Since we are only concerned with the local physics near the singularities, the manifolds can be taken to be non compact Calabi-Yau spaces.

The singularities we want to consider comprise generalisations of the gauge theory of D3-branes probing a conifold singularity, which was derived in [15]. Such hyperquotient singularities can be obtained as orbifolds of the aforementioned conifold singularity $\mathcal{C}$, and so are of the form $\mathcal{C} / \Gamma$, where $\Gamma$ is a discrete symmetry group the conifold admits [47]. The gauge theory of D3-branes on $\mathcal{C} / \Gamma$ is then defined by a similar quotient of the theory on $\mathcal{C}$ as described in 1.3.1.

The effects of T-duality and mirror symmetry of type II string theory will be discussed. The mirror transformation to the mirror geometry $\tilde{\mathcal{M}}$ will be understood precisely in the spirit of [48], namely by performing three T-dualities around isometric directions of the geometric singularity. All singularities we are interested in do have a toric description, so one can equivalently apply the local mirror map in the toric language [49, 50]. Nevertheless the first point of view will be more useful here, since it allows to follow the action of mirror symmetry on the stage of the D-branes. 


\subsection{Toric Description of Singularities}

One is now in place to introduce toric geometry [51], as a tool for treating more complicated singularities. A toric variety $\mathcal{M}$ is given by a fan in the $\mathbb{N}=\mathbb{Z}^{d}$-lattice. Every generating element $\vec{v}_{i}$ of the lattice determines one divisor $D_{i}$. The set of divisors split

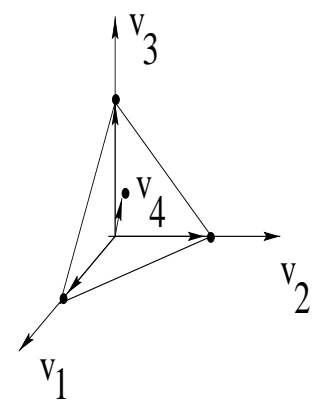

Fig. 2.1: Toric data into the principal divisors generated by functions and exceptional ones. Functions can be seen as formal linear combinations of elements in the lattice $\mathbb{M}$, which is the dual lattice of $\mathbb{N}$. The principal divisors are the images of the map $\alpha$.

$$
\begin{aligned}
\alpha: \mathbb{M} & \rightarrow \bigoplus_{i=1}^{d+n} \mathbb{Z} \cdot D_{i} \\
\vec{m} & \mapsto \sum_{i=1}^{d+n}<\vec{m}, \vec{v}_{i}>D_{i}
\end{aligned}
$$

Then one can talk about linear equivalence of divisors. This is defined in the following way: Two divisors are equivalent, iff their difference is a principal divisor. Equivalence classes of divisors are classified by the divisor class group $A_{d-1}(\mathcal{M})$ :

$$
0 \longrightarrow \mathbb{M} \stackrel{\alpha}{\longrightarrow} \bigoplus_{i=1}^{d+n} \mathbb{Z} \cdot D_{i} \stackrel{\operatorname{deg}}{\longrightarrow} A_{d-1}(\mathcal{M}) \longrightarrow 0
$$

This group contains the complete information about the grading of the divisors with respect to the torus action. This very formal approach can be translated into the language of linear sigma models [52,53]. In this picture one constructs a linear sigma model whose moduli space will be a non compact Calabi-Yau manifold $\mathcal{M}$, which allows for a toric description. Every vector $\vec{v}_{i}$ corresponds to a matter multiplet in the sigma model which is called $x_{i}$ [54]. Following physical conventions, we will denote these matter fields by $A_{i}$ and $B_{i}$ later on. Since $\mathbb{N}$ is only d-dimensional, there are $n$ relations between the $d+n$ vectors which we will write in the form

$$
\sum_{i=1}^{d+n} Q_{i}^{a} \vec{v}_{i}=0, \quad a=1, \ldots, n .
$$

They are given as the cokernel of $\alpha$ and define the images of each single divisor inside $A_{d-1}(\mathcal{M})$. The $Q_{i}^{a}$ defines a weight for the $x_{i}[55]$ which transform like $x_{i} \rightarrow \lambda^{Q_{i}^{a}} x_{i}$, $a=1, \ldots, n$, where $\lambda$ takes values in the complexified gauge group. From the physical point of view the $Q_{i}^{a}$ should be interpreted as the charges of the matter fields under the $n U(1)$ 's. Expressing the constraints of eq. (2.1) in terms of invariant polynomials one finds the equations defining the toric variety ( the singularity ). 
From the sigma model perspective the same result can be obtained by requiring a D-flatness conditions,

$$
\sum_{i=1}^{d+n} Q_{i}^{a}\left|x_{i}\right|^{2}=r_{a}, \quad a=1, \ldots, n .
$$

from which $\mathcal{M}$ can be obtained as the space of solutions to (2.2), up to the identifications imposed by gauge symmetry (thus $\mathcal{M}$ denotes the manifold obtained by smoothing out the singularity ). The number of independent Fayet-Iliopoulos parameters $r_{a}$ appearing in eq. (2.2), or equivalently the number of $U(1)$ factors, will equal the Hodge number $h^{1,1}(\mathcal{M})$, the blowup modes of the Kähler structure. It is this number, and the charges of the various matter multiplets that toric geometry encode.

For the visualisation of the $\mathbb{N}$-lattice a slight simplification occurs when $\mathcal{M}$ is a (noncompact) Calabi-Yau manifold. The Calabi-Yau condition states that the canonical class is trivial, i.e. there should exist an element $\vec{m}$ of the $\mathbb{M}$-lattice, so that

$$
K= \pm \sum_{i=1}^{n} D_{i}= \pm \sum_{i=1}^{n}<\vec{m}, v_{i}>\cdot D_{i} .
$$

This leads to $\left\langle\vec{m}, v_{i}\right\rangle=1$, which is nothing than the Hessian normal form. It states that all vectors $v_{i}$ should lie in the plane perpendicular to $\vec{m}$ and with the distance one to the origin. Since in all our examples the lattice $\mathbb{N}$ is 3-dimensional, toric singularities can be described by planar diagrams, only.

\subsubsection{Conifold}

A simple isolated singularity a three dimensional Calabi-Yau manifold can develop is

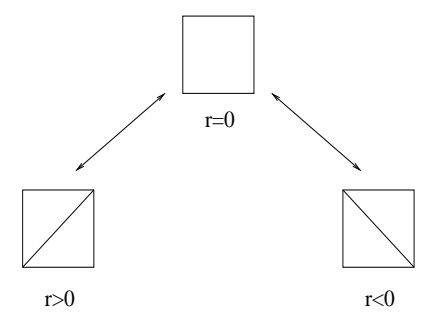

Fig. 2.2: Two small resolutions the conifold. The toric diagram (fan) is generated by the four vectors $v_{1}=(0,0,1), v_{2}=(1,0,1)$, $v_{3}=(0,1,1)$ and $v_{4}=(1,1,1)$ in $\mathbb{C}^{3}$ and the single relation can be set into an equation:

$$
\mathcal{C}: \quad x y-u v=0
$$

There are alternatives to smooth out the singularity, resulting in topologically distinct spaces. Small resolution replaces the singular point by a $\mathbb{C P}^{1}$, thereby changing the Kähler structure. Different ones are related by a flop (see Fig. 2.2). $|r|$ is the size of the $\mathbb{C P}^{1}$ and the sign denotes which one (cf. eq. (2.2)). The resulting spaces have $h^{1,1}=1, h^{2,1}=0$. A second possibility is to resolve the singularity by adding a constant $\epsilon$ to the right hand side of eq. (2.3), which changes the complex structure. After the deformation, $h^{1,1}=0, h^{2,1}=1$. 


\subsubsection{Orbifolds}

The toric diagram of the orbifold $\mathcal{O}_{k l}=\mathbb{C}^{3} / \mathbb{Z}_{k} \times \mathbb{Z}_{l}$ can be obtained starting from a toric diagram containing just three vectors $\vec{v}_{1}=(0,0,1), \vec{v}_{2}=(1,0,1), \vec{v}_{3}=(0,1,1)$ in an integral lattice $\mathbb{N}$, which gives a toric variety homeomorphic to flat space $\mathbb{C}^{3}$, and then refine the lattice to $\mathbb{N}^{\prime}$, as in Fig. 2.3 by including the vectors $\vec{e}_{k}=\left(\vec{v}_{3}-\vec{v}_{1}\right) / k$ and $\vec{e}_{l}=\left(\vec{v}_{2}-\vec{v}_{1}\right) / l$. The map from the toric variety in $\mathbb{N}$ to the one living in $\mathbb{N}^{\prime}$ is

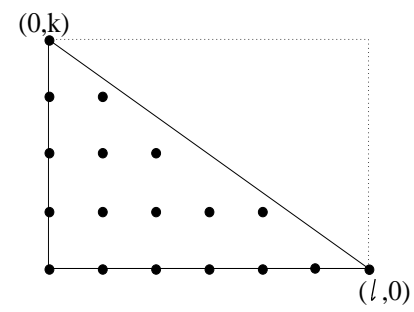
one to one provided one includes discrete identifications on the three matter fields $A_{i}, i=1,2,3$,

$$
A_{1} \sim e^{-\frac{2 \pi i}{l}} A_{1}, \quad A_{2} \sim e^{\frac{2 \pi i}{l}} A_{2}, \quad A_{3} \sim A_{3},
$$

and

$$
A_{1} \sim e^{-\frac{2 \pi i}{k}} A_{1}, \quad A_{2} \sim A_{2}, \quad A_{3} \sim e^{\frac{2 \pi i}{k}} A_{3} .
$$

The number of Kähler structure deformations is just Fig. 2.3: Toric diagram of $\mathcal{O}_{k l}$. the number of independent points in the toric diagram, and this number will clearly depend on whether $(k, l)$ are coprime or not, since the number of points on the diagonal is $\operatorname{gcd}(k, l)+1$. The complex structure deformations can be obtained from the deformation of the equations derived in appendix B.

\subsubsection{Hyperquotient Singularities}

Taking a quotient by a discrete group action of a hypersurface singularity like $\mathcal{C}$ one obtains what are called hyperquotient singularities. Both can be treated easily in the language of toric geometry. One is up to producing orbifolds of the conifold, $\mathcal{C} / \Gamma$. Let us take $\Gamma=\mathbb{Z}_{k} \times \mathbb{Z}_{l}$. So, start with the conifold $\mathcal{C}$ (Fig. 2.2), but refine the cone spanned by

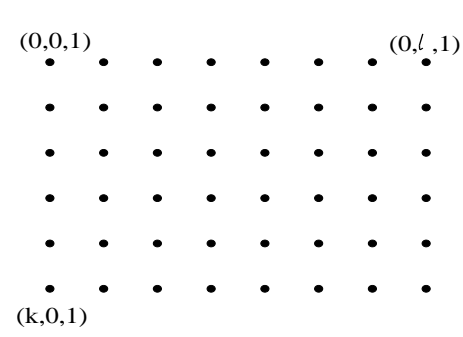

Fig. 2.4: Orbifolded conifold $\mathcal{C}_{k, l}$. $\vec{v}_{1}, \ldots, \vec{v}_{4}$ by adding two vectors, $\vec{e}_{k}=\left(\frac{1}{k}, 0,0\right)$, and $\vec{e}_{l}=\left(0, \frac{1}{l}, 0\right)$. The resulting toric diagram (cf. Fig. 2.4) "looks" the same as that for the conifold $\mathcal{C}$, except for the fact that it lives in a finer lattice. This, as explained above, results in the following identifications. With $\vec{e}_{k}=\left(\vec{v}_{2}-\vec{v}_{1}\right) / k$ and $\vec{e}_{l}=\left(\vec{v}_{3}-\vec{v}_{1}\right) / l$ one finds that the group $\Gamma$ acts by

$$
\begin{aligned}
& A_{1} \sim e^{-\frac{2 \pi i}{k}} A_{1}, \quad B_{1} \sim e^{\frac{2 \pi i}{k}} B_{1}, A_{2} \sim A_{2}, \quad B_{2} \sim B_{2}, \\
& A_{1} \sim e^{-\frac{2 \pi i}{l}} A_{1}, \quad B_{1} \sim B_{1}, \quad A_{2} \sim A_{2}, \quad B_{2} \sim e^{\frac{2 \pi i}{l}} B_{2} .
\end{aligned}
$$
Equivalently on $x y=u v$, one identifies $x \sim x, y \sim y, u \sim e^{-\frac{2 \pi i}{k}} u, v \sim e^{\frac{2 \pi i}{k}} v$, and $x \sim e^{-\frac{2 \pi i}{l}} x$, $y \sim e^{\frac{2 \pi i}{l}} y, u \sim u, v \sim v$. In terms of $\Gamma$ invariant coordinates (for details see appendix B) the orbifolded conifold $\mathcal{C}_{k, l}$ is given by the equations:

$$
\mathcal{C}_{k, l}: \quad x^{\prime} y^{\prime}=z^{l}, \quad u^{\prime} v^{\prime}=z^{k} .
$$


In the following we usually neglect the primes on the coordinates.

\section{Resolution by Blowing Up}

Toric geometry has equipped us with a means of blowing up the singularity. First let us look at the orbifolded conifold $\mathcal{C}_{k l}$. There are still only four vectors defining the diagram which were inherited from the conifold. There is a single relation between them, and thus a single Kähler class but this is insufficient to smooth out $\mathcal{C}_{k, l}$. However, due to the fact that the lattice is finer, there exist lattice points within the rectangle, these are all the points $\vec{v}_{i, j}=(i, j, 1), 0 \leq i \leq k, 0 \leq j \leq l$. One can add these points to the toric diagram. In the language of linear sigma models, the effect is to add more matter fields, but also more $U(1)$ factors, and thus more Fayet-Iliopoulos parameters. Clearly, the resolved manifold will have $h^{1,1}\left(\mathcal{C}_{k, l}\right)=(k+1)(l+1)-3$, which is the total number of linear dependent vectors within the diagram. Thus starting from $\mathcal{C}_{k, l}$, with $k, l$ sufficiently large, by performing partial resolutions one obtains essentially any other toric singularity ${ }^{*_{1}}$. Adding or subtracting one of the boundary points of the diagram changes $h^{1,1} \rightarrow h^{1,1}-1$.

\section{Resolution by Deformation of the Equation}

The orbifolded conifold $\mathcal{C}_{k l}$ can be deformed into a smooth space by modifying the defining equation as:

$$
x y=\prod_{i=1}^{k}\left(z-w_{i}\right), \quad u v=\prod_{j=1}^{l}\left(z-w_{j}^{\prime}\right) .
$$

One of these parameters can be set to 1 by shifting $z$, so one is left with $k+l-1$ parameters. This gives $h^{2,1}\left(\mathcal{C}_{k l}\right)=k+l-1$.

\subsubsection{Generalised Conifold}

By partial resolution of $\mathcal{C}_{k}:=\mathcal{C}_{k 1}$, one can obtain the generalisation of a conifold, with

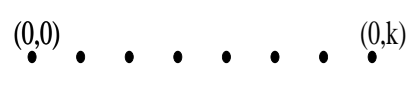

$(1, l)$

Fig. 2.5: Generalised conifold $\mathcal{G}_{k l}$. only $k+l-1$ Kähler structure deformations and defining equation:

$$
\mathcal{G}_{k l}: \quad x y=u^{k} v^{l}
$$

The defining equation of $\mathcal{G}_{k l}$ can be deformed into

$$
x y=\sum_{i, j=0}^{k, l} m_{i j} u^{i} v^{j} .
$$

\footnotetext{
${ }^{* 1}$ These singularities have been introduced in the physics literature for the description of gauge theories in [56].
} 
This time we see $h^{2,1}\left(\mathcal{G}_{k l}\right)=(k+1)(l+1)-3$ complex structure deformations $m_{i j}$, since one can eliminate two of the parameters by shifting $u, v$ and another one by rescaling the defining equation.

Clearly, in this notation $\mathcal{G}_{k k} \equiv \mathcal{C}_{k}$.

\subsection{Local Mirror Symmetry \& dual Geometries}

Toric geometry is well adopted to discussing mirror symmetry as well. We will review it here very briefly, only. Mirror symmetry exchanges the Kähler structure parameters with the complex structure parameters. Now, to understand the mirror map, one first needs to know something about the complex structure moduli space. How is the complex structure encoded in the equation of the manifold? The answer is as follows: the coefficients of the monomials appearing in the defining equation are coordinates on the complex structure moduli space. What they parameterise are the "sizes" of various three-cycles (i.e. the periods of the holomorphic three form) and the metric on the moduli space. The periods, (and therefore the metric - the moduli space has special geometry structure) can be derived directly as a solution to a system of differential equations (Picard-Fuchs equations). The main point is that the differential equations depend solely on the relationships between the monomials in the defining equation and nothing else. Given the toric manifold $\mathcal{M}$ (assuming a completely smooth space here, with all the possible blowups performed), relations between the vectors in the toric diagram of $\mathcal{M}$

$$
\sum_{i=1}^{n+d} Q_{i}{ }^{a} \vec{v}_{i}=0, \quad a=1, \ldots n
$$

map to relationships between the monomials in the defining equation of $\tilde{\mathcal{M}}$, the mirror of $\mathcal{M}$, given by

$$
\tilde{\mathcal{M}}: \quad \sum_{i} a_{i} m_{i}=0
$$

where $a_{i}$ are coefficients, and $m_{i}$ monomials, the monomials must satisfy

$$
\prod_{i=1}^{n+d} m_{i}^{Q_{i}^{a}}=1, \quad a=1, \ldots n .
$$

Any solution to these equations (and in general there are more than one) will represent the same complex structure. Note that there are $n+d$ monomials with $n$ relations between them. Together with the hypersurface equation, this gives a $d-1$ dimensional 
manifold, but the homogeneity of the monomial relations will allow us to remove one more. The mirror will naively have $d-2$ dimensions. This is not a problem, rather an artifact of the fact that local mirror symmetry is encoding all the information about the complex structure of the mirror, and nothing but. One can fix the "dimensionality" of the local mirror by adding quadratic pieces, as this will not influence the complex structure moduli space.

Let us briefly show how this works for the two examples we will be concerned within this work ${ }^{*_{2}}, \mathcal{C}_{k l}$ and $\mathcal{G}_{k l}$. Consider first the blowup of $\mathcal{C}_{k l}$. We want to interpret the same diagram Fig. 2.4 as defining the complex structure of the mirror. Assigning the vector $(i, j, 1)$ to a monomial $u^{i} v^{j}$ clearly eq. $(2.9)$ is satisfied for all the relations by construction. The defining equation for the mirror of $\mathcal{C}_{k l}$ hence becomes according to eq. (2.8):

$$
\sum_{i, j=0}^{k, l} m_{i j} u^{i} v^{j}=0
$$

Note that $m_{i j}$ now refers to the coefficients. After adding irrelevant quadratic pieces and an obvious manipulation $x^{\prime 2}+y^{\prime 2}=\left(x^{\prime}+i y^{\prime}\right)\left(x^{\prime}-i y^{\prime}\right)=: x y$ the equation can be written as

$$
x y=\sum m_{i j} u^{i} v^{j}
$$

which is nothing but the deformation of $\mathcal{G}_{k l}$. Having established that the blowup of $\mathcal{C}_{k l}$ is mirror to the deformation of $\mathcal{G}_{k l}$ one can find another dual pair by following our geometries through a conifold transition. One should find that the blowup of $\mathcal{G}_{k l}$ is the mirror of the deformation of $\mathcal{C}_{k l}$. Let us see how this works. As before mapping the lattice points of Fig. 2.5 to monomials the equation of the mirror is:

$$
\prod_{i=1}^{k}\left(z-w_{i}\right)+t \prod_{j=1}^{l}\left(z-w_{j}^{\prime}\right)=0 .
$$

Because $t$ appears only linearly this encodes the same complex structure as

$$
x y=\prod_{i=1}^{k}\left(z-w_{i}\right), \quad u v=\prod_{j=1}^{l}\left(z-w_{j}^{\prime}\right),
$$

where we take the freedom to add quadratic pieces again. But this is indeed the deformation of $\mathcal{C}_{k l}$ as presented in eq. (2.5). The quintessence of this discussion is that $\mathcal{C}_{k l}$ and $\mathcal{G}_{k l}$ form a mirror pair.

\footnotetext{
${ }^{*} 2$ These examples and many more along these lines have been recently analysed in great detail in [57].
} 


\subsection{The Gauge Theories}

Having introduced the geometric backgrounds we will now remind of the gauge theories, living on the worldvolume of $n$ D3-branes and probing the singular geometries. The simplest type of singularities is the orbifold singularity $\mathcal{O}_{k l}$. The gauge theory of which was studied in 1.3.1. There one founds a

$$
S U(n)^{k l}
$$

gauge theory with three types of chiral bifundamental multiplets $H_{i, j ; i+1, j}, V_{i, j ; i, j+1}$ and $D_{i+1, j+1 ; i, j}$ in each gauge group and a cubic superpotential

$$
W=\sum_{i, j} H_{i, j ; i+1, j} V_{i+1, j ; i+1, j+1} D_{i+1, j+1 ; i, j}-\sum_{i, j} V_{i, j ; i, j+1} H_{i, j+1 ; i+1, j+1} D_{i+1, j+1 ; i, j} .
$$

In [15] the field theory of $n$ D3-branes at the conifold singularity was derived. After an orbifold projection the field theory of the orbifolded conifold $\mathcal{C}_{k l}$, eq. (2.4), is given by [16] the $N=1$ supersymmetric gauge theory with gauge group

$$
S U(n)^{k l} \times S U(n)^{k l}
$$

and matter fields $\left(A_{1}\right)_{i+1, j+1 ; I, J},\left(A_{2}\right)_{i, j ; I, J},\left(B_{1}\right)_{I, J ; i, j+1},\left(B_{2}\right)_{I, J ; i+1, j}$, all in a bifundamental representations of the gauge groups as indicated by the indices. Here we are using a highly redundant notation. First we distinguish the gauge groups by taking small Latin letters for the first and big Latin letters for the second $(i, I=1 \ldots k$ and $j, J=1 \ldots l)$. The fields are indexed by pairs. The first pair denotes a fundamental the second an antifundamental representation of the appropriate group.

$$
\begin{aligned}
& \left(A_{1}\right)_{i+1, j+1 ; I, J} \quad\left(\square_{i+1, j+1}, \bar{\square}_{I, J}\right) \\
& \left(A_{2}\right)_{i, j ; I, J} \quad\left(\square_{i, j}, \quad \square_{I, J}\right) \\
& \left(B_{1}\right)_{I, J ; i, j+1} \quad\left(\square_{i, j+1}, \square_{I, J}\right) \\
& \left(B_{2}\right)_{I, J ; i+1, j} \quad\left(\square_{i+1, j}, \square_{I, J}\right)
\end{aligned}
$$

In addition there will be a quartic superpotential

$$
\begin{aligned}
W= & \sum_{i, j}\left(A_{1}\right)_{i+1, j+1 ; I, J}\left(B_{1}\right)_{I, J ; i, j+1}\left(A_{2}\right)_{i, j+1 ; I, J+1}\left(B_{2}\right)_{I, J+1 ; i+1, j+1} \\
& -\sum_{i, j}\left(A_{1}\right)_{i+1, j+1 ; I, J}\left(B_{1}\right)_{I, J ; i+1, j}\left(A_{2}\right)_{i+1, j ; I+1, J}\left(B_{2}\right)_{I+1, J ; i+1, j+1} .
\end{aligned}
$$

The field theory of the third singularity, the generalised conifold eq. (2.6), does have gauge group $S U(n)^{k+l}$ with bifundamental matter according to the rules in [16] and quartic superpotential. 
This way the orbifold gauge theories will have $3 n$ matter fields per gauge group and cubic superpotentials, leaving us with a finite theory. The conifold gauge theories have $2 n$ matter fields per gauge group and quartic superpotentials. These theories are nonfinite but flow to a fixed line parameterised by a marginal operator in the IR. They give rise to conformal field theories and have a dual $A d S$ description. Related topics will be discussed in chapter 5 .

\subsection{The associated Brane Picture}

In this section we would like to discuss the brane configurations which are T-dual to the singularities introduced in section 2.1. There are two configurations we are going to consider, for one the standard Hanany-Witten type of brane setup [36, 58], where D4branes (living in 01236) are stretched in between NS5 and NS5'-branes, former living along 012345 and latter along 012389.

\begin{tabular}{|c|c|c|c|c|c|c|c|c|}
\hline NS5 & 0 & 1 & 2 & 3 & 4 & 5 & & \\
\hline NS5' & 0 & 1 & 2 & 3 & & & 8 & 9 \\
\hline D4 & 0 & 1 & 2 & 3 & & 6 & & \\
\hline
\end{tabular}

Tab. 2.1: Interval

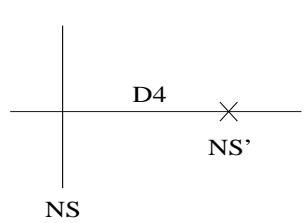

NS

The rotation is necessary in order to break SUSY down from 8 to 4 supercharges. In order to have a supersymmetric theory from D4-branes on the interval all the NS5 and NS5'-branes have to be at the same position in the 7 direction. Separations along the 7 direction would be interpreted as Fayet-Iliopoulos terms or baryonic branches in the gauge theory and effectively leads to a breaking of the gauge group we want to see. Similarly we should require all the NS5-branes to have the same position in 89 and all the NS5'-branes to have the same position in 45 space. They are separated along the 6 direction building the intervals, along which the D4-branes stretch. Now let us take the interval to be compact. The corresponding $T_{6}$-duality was first discussed in $[16,38]$ and is very similar to the case explained in section 1.2.3. The effect of the transformation is to convert the blowup of the generalised conifold $\mathcal{G}_{k l}$ into $k$ NS5 and $l$ NS5'-branes separated along $x_{6}$ (the interval). By this T-duality D3-brane probes are transformed into D4-branes stretched between the NS5/NS5'-branes. We will henceforth refer to it as $T_{6}=: T_{I}$ for interval.

The second kind of brane setup we are going to consider are the so called brane boxes [5], which are a straightforward generalisation of the interval theories. The brane box is a rectangle bounded by NS5 and NS5' branes with a D5-brane suspended on it. This can be achieved by the same NS5 and NS5'-branes as above but now all branes have to be located at the same 67 position, closing the intervals. 


\begin{tabular}{|c|c|c|c|c|c|c|c|c|}
\hline NS5 & 0 & 1 & 2 & 3 & 4 & 5 & & \\
\hline NS5' & 0 & 1 & 2 & 3 & & & 8 & 9 \\
\hline D5 & 0 & 1 & 2 & 3 & 4 & & 8 & \\
\hline
\end{tabular}

Tab. 2.2: The Box

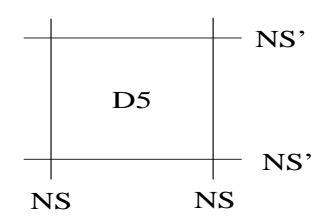

We can open up the boxes by separating the NS5 and NS5'-branes along their 48 directions $^{*_{3}}$. We still want to keep the 5 and 9 positions equal in order to preserve supersymmetry of the suspended probes. Deformations along these directions are again Fayet-Iliopoulos terms in the gauge theory, which are reinterpreted as baryonic branches after freezing out the diagonal $U(1) \mathrm{s}$. The corresponding T-duality was introduced in [35] and can be performed along the compact directions $x^{4}$ and $x^{8},\left(T_{48}=T_{4} T_{8}\right)$. It transforms the blowup of the orbifolded conifold $\mathcal{C}_{k l}$ into a box of $k$ NS5 and $l$ NS5'-branes again. But now the D3-probes will become D5-branes, which fill the compact $x^{4}-x^{8}$ directions of the brane box. We will henceforth refer to it as $T_{B}$ for box (cf. section 1.2.3).

Combining the two, that is doing $T_{\text {Mirror }}=T_{468}$ we actually performed the local mirror symmetry transformation of section 2.2. Let us forget for a moment about the D-brane probes altogether. That is, we want to study the map of the singular geometry into a configuration of NS5/NS5'-branes. Actually it turns out to be easier to start with the NS5/NS5'-brane configurations, where it is clear what is meant by the 4,6 and 8

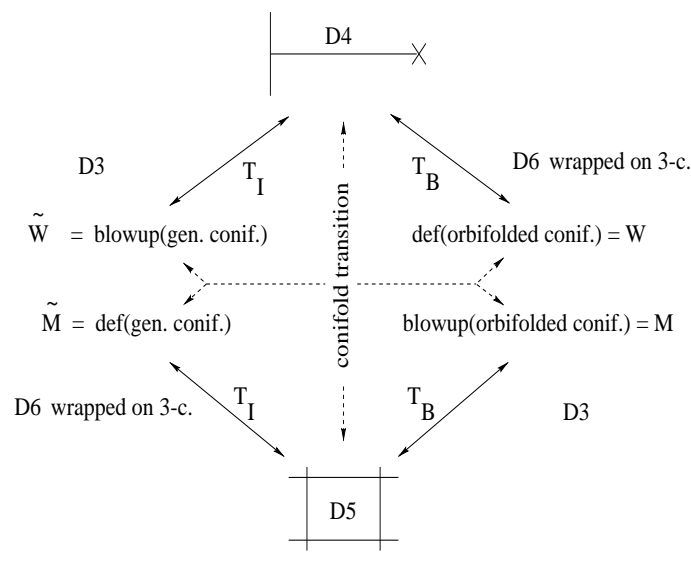

Fig. 2.6: The proposed picture. direction. Performing $T_{B}=T_{48}$ and $T_{I}=T_{6}$ respectively we found in section 2.2 two geometries, which are local mirrors of each other. Therefore one obtains

- The blowup of the generalised conifold is $T_{I}$ dual to NS5/NS5'-branes separated along 67 (the interval). These are in turn $T_{B}$ dual to the mirror, the deformation of the orbifolded conifold.

- Similarly the blowup of the orbifolded conifold will $T_{B}$ dualise into a box and then $T_{I}$ dualise in the mirror, the deformation of the generalised conifold.

${ }^{*} 3$ This differs from the notation in [5], where the boxes were taken to live in the 46 space. This deviation is nevertheless necessary, since it is crucial, that box and interval can be realized by the same set of NS5 and NS5'-branes. 
Indeed these two transformations are related by a conifold transition, that is bringing together the NS5/NS5'-branes on the interval and then separating them along 4589 instead corresponds to blowing down the 2-cycles and opening up the 3-cycles of the deformed conifold (and vice versa for the orbifolded conifold).

\subsubsection{The Brane Box and its both T-dual Geometries}

As yet discussed in section 2.2, it is important to distinguish whether one studies the deformation or the blowup of the singularity under investigation. The corresponding parameters can be found in the brane picture as well. If the dual is 'pure brane', i.e. consists only of branes in flat space, this interpretation will be solely in terms of NS5/NS5'-brane positions and, as will be established later, on brane shapes. Otherwise some of the parameters encode blowups of the non-trivial background geometry. Here we want to consider only the case, where the dual is a 'pure brane'.

In general $m_{i}=\left(x^{8}, x^{9}\right), m_{j}^{\prime}=\left(x^{4}, x^{5}\right)$ denote the positions of the $k$ NS5 and $l$ NS5'branes respectively in $x^{4,5,8,9}$, and $w_{i}=\left(x^{6}, x^{7}\right), w_{j}^{\prime}=\left(x^{6}, x^{7}\right)$ the positions in the other two directions.

If one considers the "brane box", one has to choose all the $w_{i}$ and $w_{j}^{\prime}$ equal to zero.

- $\mathbf{T}_{\mathbf{B}}$ : The $T_{B}$-dual space $\mathcal{M}$ is the orbifolded conifold $\mathcal{C}_{k, l}$ of eq. (2.4), where $k, l$ are numbers of NS5 and NS5'-branes. The $x^{4}, x^{8}$ separations of the branes must map into B-fluxes through 2-cycles of $\mathcal{C}_{k, l}$. We must therefore identify the $m_{i}, m_{j}^{\prime}$ from above with deformations of the Kähler structure. Deformations of the Kähler structure do not influence the complex structure, so the $m_{i}$ and $m_{j}^{\prime}$ will not be visible in the defining equations. Having identified $m_{i}, m_{j}^{\prime}$ as the Kähler structure parameters, $w_{i}$ and $w_{j}^{\prime}$ are identified as complex structure parameters. But they are frozen, since turning them on would destroy the box structure.

For definiteness take IIB theory compactified on $\mathcal{C}_{k l}$. $T_{B}$ duality takes us back to type IIB with NS5-branes. In this case Kähler structure parameters, that is the 2-sphere sizes, sit in hypermultiplets, which contains four real scalars. The other scalars in this multiplet are the NS-NS B-flux the R-R B-flux and the R-R 4-form-flux through the sphere. Latter is a 2 -form in $4 \mathrm{~d}$, which can be dualised into a scalar. The 2-sphere size and the NS-NS B-flux are the complexified Kähler parameter which map to $m_{i}$ and $m_{j}^{\prime}$ under $T_{B}$. In the brane box the two other scalars come from Wilson lines of the NS5-brane world volume gauge fields in 45 and 89 which pair up in hypermultiplets with $m_{i}$ and $m_{j}^{\prime}$ respectively.

Note however the little puzzle. The orbifolded conifold $\mathcal{C}_{k l}$ has $(k+1)(l+1)-3$ Kähler structure parameters $m_{i j}$ (see subsection 2.1.3) which can be turned on to smooth out $\mathcal{C}_{k l}$. Only $k+l-2$ have been realized in terms of the relative brane positions $m_{i}$ and 
$m_{j}^{\prime}$. So where are the $k l$ hypermultiplets in the brane box skeleton, which one expects? They sit at the $k l$ intersections! Strings stretching from NS5 to NS5'-branes give rise to precisely these hypermultiplets (see Fig. 2.7) [45, 59].

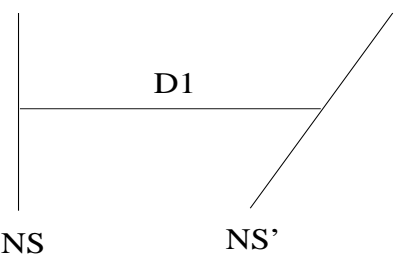

Fig. 2.7

Turning on vevs for the two scalars corresponding to 2-sphere sizes and NS-NS B-fluxes resolves the intersection of the NS5 and NS5' into a smooth object, a little 'diamond'. For non-zero B-fields this diamond will open up in the 48 plane, for 2-sphere sizes in the 59 plane. This interpretation will become more suggestive after discussing $T_{I}$ on this configuration and once we start discussing the D3-brane probes.

In the geometry the 2-spheres give rise to strings from wrapping D3-branes around them. How do we see them in the NS5 box skeleton? The D3 branes on the $k+l-2$ spheres from the curves of singularities correspond to D3-branes living in the boxes (or better in whole stripes). The additional $k l$ strings must now correspond to D3-branes in the diamonds. We will indeed see that the diamonds allow for such a configuration.

Of course the same story can be repeated in type IIA. Here the diamonds will correspond to matter on the intersection of type IIA NS5-branes, this time sitting in a vector multiplet. Again the 2 scalars correspond to the $k l$ sizes and B-fluxes of the corresponding 2 -spheres. Instead of the two additional scalars in the hypermultiplet we this time see a vector from the R-R 3-form on the sphere. In the brane language the Wilson lines of the NS5-brane gauge field have to be substituted by Wilson lines of the (2,0) 2-form field, again giving rise to vectors.

- $\mathbf{T}_{\mathbf{I}}=\mathbf{T}_{\mathbf{6}}$, T-duality to $\tilde{\mathcal{M}}$. What happens now is as follows. Since we did a $T_{6}$ duality, $x^{6}$ separations will become the B-fields. Thus, now the $w_{i}$ (which had to be put to zero since we are discussing a box) parameters are Kähler structure deformations, while the non-zero $m_{i j}$ now should show up as complex structure deformations.

The dual geometry will be described by an equation whose parameters, the complex structure deformations, must be $m_{i j}$. Let us first study the situation where the vevs of the scalars in the hypermultiplets living at the intersections are zero. In this case the $\mathbb{C}^{*}$ fibration must degenerate over the NS5 and NS5' positions $m_{i}, m_{j}^{\prime}$, but in an independent way, since the branes are orthogonal - it must contain two curves of singularities $A_{m-1}$, 
and $A_{n-1}$ corresponding to NS5 and NS5'-branes. There is one such equation for generic values of $m_{i}$ 's

$$
\tilde{\mathcal{M}}: \quad u v=\prod_{i=1}^{k}\left(z-m_{i}\right) \prod_{j=1}^{l}\left(w-m_{j}^{\prime}\right)
$$

The curve contains $k l$ conifold singularities located at $z=m_{i}$ and $w=m_{j}^{\prime}$ corresponding to the fact that all the hypermultiplets at the intersections where turned off.

Let us jump ahead and let us realize $\tilde{\mathcal{M}}$ directly as the mirror of $\mathcal{M}$. Performing the local mirror map we obtain:

$$
\tilde{\mathcal{M}}: \quad u v=\sum_{i=0}^{k} \sum_{j=0}^{l} m_{i j} z^{i} w^{j} .
$$

By now the T-dual interpretation of this more general space should be clear. It describes a single NS5-brane wrapping a curve

$$
\Sigma: \quad 0=\sum_{i=0}^{k} \sum_{j=0}^{l} m_{i j} z^{i} w^{j} .
$$

The smoothing out of the intersections corresponds to the diamonds. For example one intersecting NS5 and NS5'-brane is described by $z w=0$. Turning on the hypermultiplet corresponds to smoothing this out to $z w=m_{00}$, as e.g. discussed in [60]. Indeed the resulting smooth curve has a non vanishing circle of radius $\left(m_{00}\right)^{1 / 2}$ as can be seen by writing it as $x^{2}+y^{2}=m_{00}$ and restrict oneself to the real section thereof, for example. This is precisely what we need: we can suspend a D3-brane as a soap bubble on the NS5 skeleton, its boundary being given by the circle. The tension of the resulting string is given by the area of the disk and hence is proportional to $m_{00}$ as expected from the dual geometry $\mathcal{M}$ (where the size of the 2 -sphere was also proportional to $m$ ). In $\tilde{\mathcal{M}}$ the same string will be given by a D4-brane on the vanishing 3 -sphere.

In the same way we can T-dualise any singularity that can be represented as a toric variety into a generalised box of NS5-branes, with a certain amount of diamonds frozen.

\subsubsection{Going to the Interval through a Conifold Transition}

One can derive a second T-dual triple of geometry T-dual brane setup and mirror geometry by studying $T_{I}$ and $T_{B}$ on the interval theory. Note that the interval theory can be directly obtained from the box by brane motions. First one moves all the NS5 and NS5'-branes on top of each other, setting all $m_{i j}$ to zero, closing all the boxes and diamonds. This is the conifold point. Now one sees that one has the choice to open up the intervals, by turning on the $w_{i}$ and $w_{j}^{\prime}$. 
We can follow this transition in the geometry as well. Let us see what it does to $\mathcal{M}$. For one we have shrunk all the 2 -spheres to zero size, putting us at the most singular point of the geometry. In addition we have put all the B-fields to zero. From there we can deform the singularity by turning on 3 -spheres to obtain $\mathcal{W}$ and this is precisely what corresponds to turning on the $w_{i}$ and $w_{j}^{\prime}$ in the brane picture. This is a conifold transition [61]. We went from the blowup of the orbifolded conifold $\mathcal{C}_{k l}$ to its deformation.

Last but not least we can study the effect on $\tilde{\mathcal{M}}$. In going to $\tilde{\mathcal{W}}$, the mirror of $\mathcal{W}$, we this time send all the 3 -spheres to zero size and then turn on blowup modes, taking us from the deformed generalised conifold $\mathcal{G}_{k l}$ to its blowup.

\subsection{Probing the Mirror Geometries}

\subsubsection{Reintroducing the Probes}

As a next step we want to introduce $n$ D3-brane probes on top of the geometry. This way one breaks the supersymmetry down to 4 supercharges and get interesting $N=1$ $4 \mathrm{~d}$ gauge theory. The deformation parameters of the singularity appear as parameters in the gauge theory, the moduli space of the gauge theory describes the motion of $n$ D3-branes on the singular space.

In principle we could take any of the four geometries introduced before, compactify type IIB on it and then put a D3-brane probe on top of the singularity. The two situations we are going to study are $n$ D3-branes on the blowup of the orbifolded conifold $\mathcal{C}_{k l}(\mathcal{M})$ and $n$ D3-branes on the blowup of the generalised conifold $\mathcal{G}_{k l}(\tilde{\mathcal{W}})$.

Performing the two T-dualities $T_{I}$ and $T_{B}$ one will find two different realizations of each of the probe theories. The background geometry will transform precisely as we discussed in the last section (see Fig. 2.6). This way

- $n$ D3-brane probes of the blowup of the generalised conifold $\tilde{\mathcal{W}}$ are $T_{I}$ dual to D4-branes on an interval defined by $w_{i}$ and $w_{j}^{\prime}$ and $T_{\text {Mirror }}$ to D6-branes wrapping 3 -cycles in $\mathcal{W}$

- $n$ D3-brane probes of the blowup of the orbifolded conifold $\mathcal{M}$ are $T_{B}$ dual to D5-branes on a box defined by $m_{i j}$ and $T_{\text {Mirror }}$ to D6-branes wrapping 3-cycles in $\tilde{\mathcal{M}}$.

One has to deal with what is usually referred to as elliptical models in the literature $[2,10]$. That is the 6 direction of the interval or the 48 direction of the box are actually compact, leaving no room for semi-infinite branes. All D-brane groups will actually be gauged. 


\subsubsection{Generalised Conifold and Interval}

First we would like to consider the gauge theory on the world volume of $n$ D3-brane probes on the blowup of a generalised conifold singularity ${ }^{*_{4}}$. This gauge theory is given e.g. in [16] and can be read off most easily in the dual brane setup we are about to describe. In the last section we have seen that this geometry is $T_{I}$ dual to NS5 and NS5'-branes on a circle, forming intervals with 67 separations given by $w_{i}$ and $w_{j}^{\prime}$, all the $m_{i j}$ being zero. As utilised in $[16,38]$ this means that the $n$ D3-brane probes turn into an elliptical model with $n$ D4-branes wrapping the circle. It is straightforward to read off the gauge theory from this according to the standard Hanany-Witten rules. Of course it agrees perfectly with the one obtained from applying a standard orbifold procedure directly on the conifold gauge theory of [15].

There is yet another realization of the same gauge theory. Performing the whole $T_{\text {Mirror }}=T_{468}$ one can turn $\tilde{\mathcal{W}}$, the blowup of the generalised conifold on which we originally put the D3-brane probes, into $\mathcal{W}$, the deformation of the orbifolded conifold. The theory with which one has to compare is that on the mirror of the D3 probe, that is a D6-brane wrapping SUSY 3-cycles in $\mathcal{W}$. But this is precisely the situation discussed in [63]. The parameters $w_{i}$ and $w_{i}^{\prime}$ in $\mathcal{W}$, given by (2.5) determine the loci in the $z$ plane where the $\mathbb{C}^{*} \times \mathbb{C}^{*}$ fibration degenerates. As found in [63] in order to have a BPS state the $w_{i}$ and $w_{i}^{\prime}$ have to align along a line in the $z$ plane. Since the $S^{1} \times S^{1}$ fibration degenerates over $w_{i}$ and $w_{i}^{\prime}$, we can regard this fibration over the interval between neighbouring $w_{i}$ and $w_{i}^{\prime}$ as a 3-cycle. In [63] it was shown that this 3-cycle is $S^{3}$ and $S^{2} \times S^{1}$ respectively, depending on whether neighbouring points are a $w, w^{\prime}$ pair or both $w$ (both $\left.w^{\prime}\right)$. In the former case one obtains a quartic superpotential, in the latter case an $N=2$ like setup. Obviously this yields the same gauge theory as the D3 probe on $\tilde{\mathcal{W}}$ and the D4-brane on the interval.

\subsubsection{D5-Branes on the Box, the modified Box Rules}

The second theory we would like to consider are $n$ D3-branes on an orbifolded conifold $\mathcal{C}_{k l}$. As shown above, the geometry dualises under $T_{B}$ into brane boxes where the NS5-brane skeleton wraps the curve in eq. (2.14). The $m_{i j}$ parameters can be associated to brane positions, while the other $k l$ parameters correspond to diamonds, that is the hypermultiplets sitting at the NS5/NS5' intersections, whose vev smoothes out the singular intersections.

The probe D3-branes turn into D5-branes living on these boxes and diamonds. Again this should in principle be a very useful duality in the sense that we can read off the associated gauge theories by using an analogue of the Hanany-Witten rules. In addition some information about the corresponding quantum gauge theory should be obtainable

\footnotetext{
${ }^{{ }^{4}}$ Similar setups have been discussed recently in [62].
} 
by lifting the setup to M-theory. In order to understand the new rules it is best to start with the easiest example, the conifold $\mathcal{C}=\mathcal{C}_{11}$, eq. (2.3), itself. This model is dual to an interval as well as a box $[16,38]$. The dual description in terms of a box is just that of a single NS5 and NS5'-brane on a square torus, as depicted in Fig. 2.8.a for a generic situation. The conifold has one blowup parameter, corresponding to the one diamond sitting at the intersection. As long as we keep the size of this 2-sphere zero, the B-flux through the sphere will correspond to the size of the diamond (cf. page 32). As we have argued in the last section, the curve describing the diamond actually supports a non-trivial $S^{1}$ on which the D5-brane can end, so the gauge theory will have two group factors, $S U(M) \times S U(M)$. The inverse gauge couplings are proportional to the area of the corresponding faces.

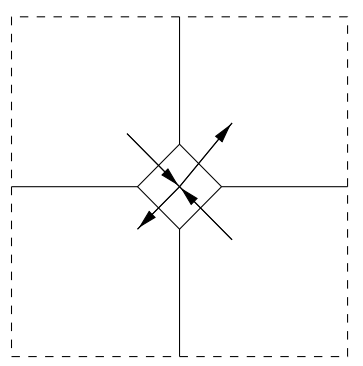

a.) generic $B$-value

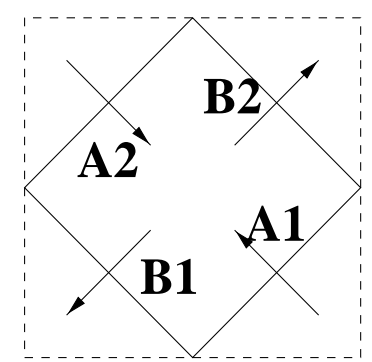

b.) maximal $B$-value

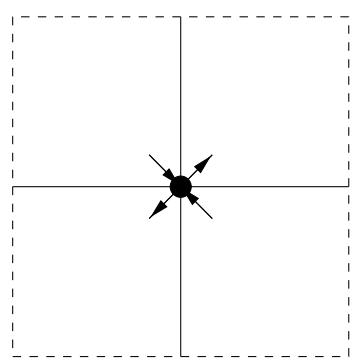

c.) $B=0 \Leftrightarrow g \rightarrow \infty$

Fig. 2.8

There is a special point, when the diamond has the same area as the other gauge group, that is the diamond occupies half of the torus. In this case we know that we have to recover the standard conifold gauge theory of $[15]^{*_{5}}$. By comparison this can easily be implemented using the simple brane rules specified in Fig. 2.8.b. We have to demand, that half of the matter multiplets we would naively expect are projected out. The transformation properties of the fields can be represented by an arrow. His orientation indicate which index of the field sits in the fundamental and which in the antifundamental representation of the gauge group. The orientation of the arrows seems quite arbitrary. Indeed we will see that the orientation can be changed and that this corresponds to performing flop transitions in the dual geometry. Indeed one can easily establish that these rules also are capable of realizing more complicated setups. Generically, the gauge theory on the $\mathcal{C}_{k l}$ orbifolded conifold has a $S U(M)^{k l} \times S U(M)^{k l}$ gauge group. In our picture the gauge group factors will correspond to the $k l$ diamonds and the $k l$ boxes respectively. Again it is easiest to compare at the point, where all gauge couplings are

\footnotetext{
${ }^{*} 5$ The reason is, that to establish the equivalence of the field theory from branes to the conifold theory one usually has to take the gauge couplings to be equal [16].
} 


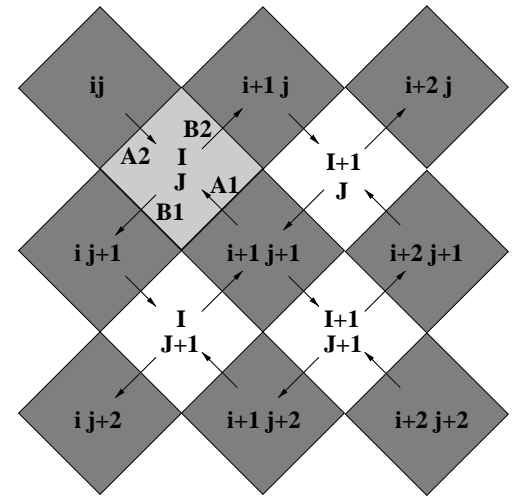

Fig. 2.9: The diamond rules at the point of maximal B-fields. equal. In this case, both the diamonds as well as the boxes degenerate to rhombes, as pictured in Fig. 2.9, where we denoted them as filled and unfilled boxes. Generalising our $A$ and $B$ fields from above we will find that the matter fields transform as

$$
\begin{array}{ll}
\left(A_{1}\right)_{i+1, j+1 ; I, J} & \left(\square_{i+1, j+1}, \square_{I, J}\right) \\
\left(A_{2}\right)_{i, j ; I, J} & \left(\square_{i, j}, \quad \square_{I, J}\right) \\
\left(B_{1}\right)_{I, J ; i, j+1} & \left(\square_{i, j+1}, \quad \square_{I, J}\right) \\
\left(B_{2}\right)_{I, J ; i+1, j} & \left(\square_{i+1, j}, \quad \square_{I, J}\right)
\end{array}
$$

which are exactly the rules expected [16] (the two sets of $k l$ gauge groups are indexed by small and capital letters respectively). This proposal can also easily deal with the situation of non-trivial identifications on the torus as discussed in [10].

In addition there will be quartic superpotential for every closed rectangle, the relative sign being given by the orientation

$$
\begin{aligned}
W= & \sum_{i, j}\left(A_{1}\right)_{i+1, j+1 ; I, J}\left(B_{1}\right)_{I, J ; i, j+1}\left(A_{2}\right)_{i, j+1 ; I, J+1}\left(B_{2}\right)_{I, J+1 ; i+1, j+1} \\
& -\sum_{i, j}\left(A_{1}\right)_{i+1, j+1 ; I, J}\left(B_{2}\right)_{I, J ; i+1, j}\left(A_{2}\right)_{i+1, j ; I+1, J}\left(B_{2}\right)_{I+1, J ; i+1, j+1}
\end{aligned}
$$

We do not expect that this picture changes when we take the sizes of box and diamond to differ. We will still see the $A$ and $B$ fields. Only the relative couplings will change and no new fields or interactions appear, since they certainly don't in the dual geometry. The singular conifold points correspond to the situations where diamonds close. From the field theory point of view this just means that we take the corresponding gauge coupling to infinity. As in the standard Hanany-Witten situation with only parallel NS5-branes this corresponds to a strong coupling fixed point with possibly enhanced global symmetry if several NS5-branes coincide.

Another interesting question to consider is to ask ourselves what happens when we blow up the spheres to finite size. This now should correspond to some mode of the diamond that "rotates" it away out of the 48 plane into the 59 plane. According to common lore this should correspond to a Fayet-Iliopoulos term in the gauge theory. We will no longer be able to support a D5-brane stretched inside the diamonds in a supersymmetric fashion, independent of their size (that is the B-field) ${ }^{*}$. Since we expect that the centre

\footnotetext{
${ }^{*} 6$ This is very similar to what happens on the interval: blowing up a sphere corresponds to moving off an NS5brane in the 7 direction. Since in order to preserve supersymmetry branes are only allowed to stretch along the 6 direction this effectively reduces the number of gauge groups (the number of intervals) by one. The 6 position of the brane we moved away (the B-field on the blown up sphere) does not affect the massless matter content anymore.
} 
of mass $U(1)$ s are frozen out as in [2], the Fayet-Iliopoulos term will be reinterpreted as a baryonic branch as usual. Especially there should exist a baryonic branch along which we reduce to the orbifold gauge theory.

Indeed as shown in [16] the gauge theories described here do have such a baryonic branch. Giving a vev to (say) all the $A_{2}$ fields will break each $S U(M)_{i j} \times S U(M)_{I J}$ pair down to its diagonal $S U(M)_{a b}$ subgroup. The remaining massless fields after the Higgs mechanism are

$$
\begin{aligned}
& D_{a+1, b+1 ; a, b}=\left(A_{1}\right)_{a+1, b+1 ; a, b} \quad\left(\square_{a+1, b+1}, \square_{a, b}\right) \\
& V_{a, b ; a, b+1}=\left(B_{1}\right)_{a, b ; a, b+1} \quad\left(\square_{a, b}, \quad \square_{a, b+1}\right) \\
& H_{a, b ; a+1, b} \quad=\left(B_{2}\right)_{a, b ; a+1, b} \quad\left(\square_{a, b}, \quad \square_{a+1, b}\right)
\end{aligned}
$$

with the remaining superpotential:

$$
W \sim \sum_{a, b} D_{a+1, b+1 ; a, b} V_{a, b ; a, b+1} H_{a, b+1 ; a+1, b+1}-\sum_{a, b} D_{a+1, b+1 ; a, b} H_{a, b ; a+1, b} V_{a+1, b ; a+1, b+1}
$$

which are precisely the box rules of [5], as claimed. Note that the diagonal $D$ fields, which from the point of view of [5] looks something obscure, are not special at all. They arise just from the fundamental $A, B$ degrees of freedom of the generalised box.

A small complication arises once we consider situations that are more involved than the conifold. For simplicity let us study the case of the $\mathbb{Z}_{2}$ orbifolded conifold. Since this can as well be thought of as the $\mathcal{G}_{22}$ generalised conifold, it has a box dual as well as an interval dual. Both of them are displayed in the sequence of figures 2.10 for various values of the B-fields. The gauge group is $S U(M)^{4}$ and one sees only 3 B-fields governing the relative sizes of the gauge couplings, since the volume of the torus produce one relation on the four couplings.
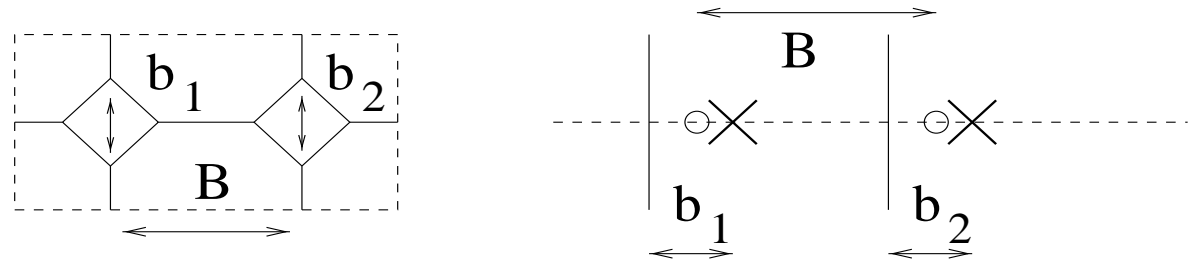

Fig. 2.10.1: Generic position of branes

According to our scenario this will correspond to one relative brane position $B$ and the sizes of two diamonds $b_{1}$ and $b_{2}$. In the interval picture $b_{1,2}$ will be the distance between $\mathrm{NS}_{i}$ and NS5' ${ }_{i}(i=1,2)$ while $B$ is the distance between the centre of masses of the two NS5/NS5' pairs, denoted as circles in Fig. 2.10.1.

Take the torus to have sides 2 and 1 and the periodic interval to have circumference 2 . Since B-fields (inverse gauge couplings) are areas on the torus and length on the interval, in these units the area of a given gauge group on the torus should have the same 
numerical value as the corresponding length on the circle (total area=total length $=2$ ). Fig. 2.10.2 shows $B=1$ and $b_{1}=b_{2}=1 / 2$. Both sides have 4 gauge groups of strength of inverse couplings $1 / 2$
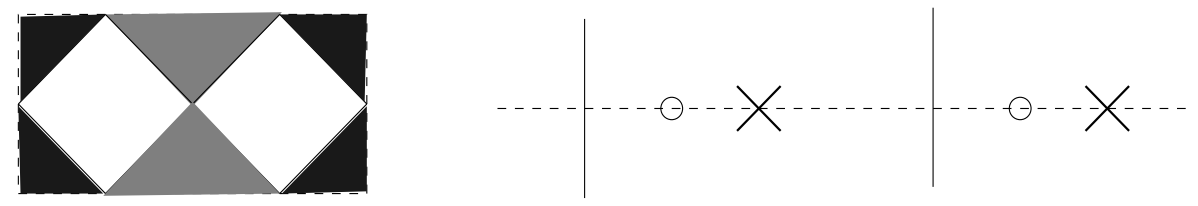

Fig. 2.10.2: All gauge couplings are equal

and therefore on both sides all gauge couplings are equal. It is very simple to find two further special points, namely the point where all B-fields are zero (the most singular point) and the point where the setup looks like two separated conifolds ( Fig. 2.10.3 ).
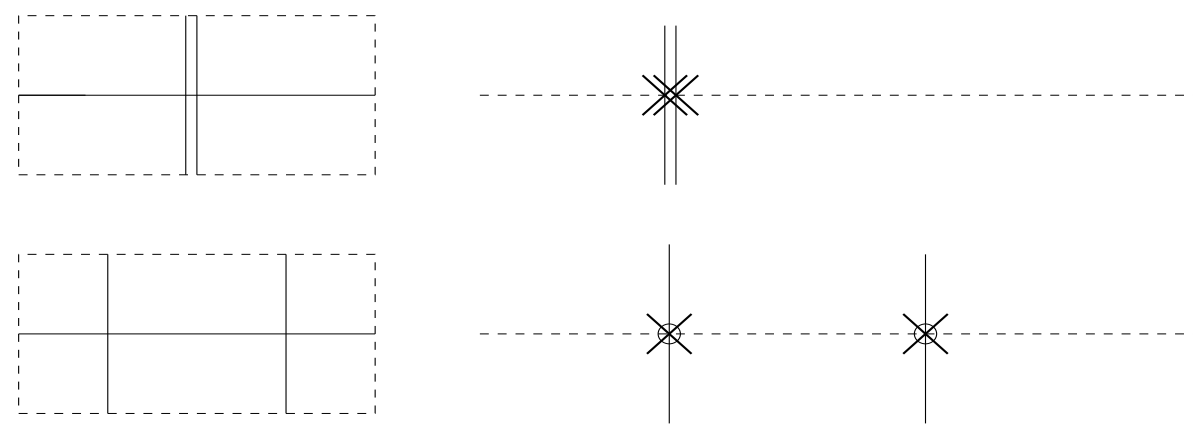

Fig. 2.10.3: Two extreme situations

Similarly for all positive values of the $b_{i}$ and of $B$ we can read off the gauge theory from the diamonds, just using the standard $A$ and $B$ fields, representing the diamonds as rhombes of area $b_{i}$. However from the interval it is clear, that we can also pass an NS5'-brane through an NS5-brane, performing Seiberg duality on the gauge theory and simultaneously changing the sign of one of the $b_{i}$ fields $[16,64]$. If we set $b_{1}=b_{2}=-1 / 2$ the picture looks the same as for $b_{1}=b_{2}=1 / 2$. The overall sign does not matter. However Fig. 2.10.4 shows a setup where the signs of the $b_{i}$ differ.
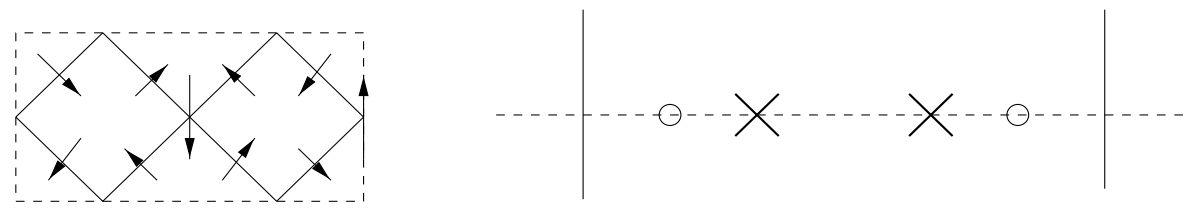

Fig. 2.10.4: Diamonds do have an orientation. 
We should assign our diamonds an orientation in order to be able to address this issue. This orientation assigns whether the $A$ or the $B$ fields point outward or inward, the other doing the opposite. The rules we have introduced are valid for the case that all orientations are equal. The situation with opposite orientations is slightly more complicated. The rules can be determined by comparing with the interval. Whenever the arrows point around the closed rectangle we write down a quartic superpotential. If diamonds with different orientation touch, we will have to introduce additional 'meson' fields with cubic superpotential (see Fig. 2.10.4). Since this inversion of orientation should correspond to Seiberg duality in the field theory, we basically found this way a realization of $N=1$ dualities in the box and diamond picture.

\subsection{Concluding Remarks}

The D3-brane probe we have been considering so far maps to a D4-brane on the interval and a D5-brane in the box respectively. We identified the corresponding gauge theories above. For a special subclass of models it turns out that one can actually perform both. These geometries are those whose toric diagram is given by two rows of $k$ points. Viewing them as orbifolded conifolds $\mathcal{C}_{k}$, their blowups turn into a box with 1 NS5' and $k$ NS5 under $T_{B}$. One can as well describe them as a $\mathcal{G}_{k k}$ generalised conifold and hence $T_{I}$ dualise them into an interval with $k$ NS5 and $k$ NS5'-branes. According to this philosophy these two ways of realizing the gauge theory should actually be mirror to each other. So one was able to turn one Hanany-Witten setup into its 'mirror branes'. Now we can try to solve these gauge theories via the lift to M-theory. Interestingly enough, the intervals lift via $S U(2)$-SLAG 2-cycles in $\mathbb{R}^{6}$ while the boxes lift via $S U(3)$-SLAG 3 -cycles $[9]$ in $\mathbb{R}^{6}$. So the information contained in a 3 -cycle should be related to those of the dual 2-cycle and vice versa. 


\section{Chapter 3}

\section{BPS M5-Branes}

Supersymmetric M5-brane solutions have received a lot of attention through the role they are playing in the description of gauge theories in string theory. It became clear, that various supersymmetric brane configurations embedded in flat spacetime capture a lot of information about the nonperturbative behaviour of supersymmetric gauge theories, which can be extracted by lifting brane configurations to 11d M-theory (more about this in chapter 4). As noted in subsection 1.1.1 the M5-brane support a host of fields, whose equations of motion describe the dynamical behaviour of the brane. If one switches off the closed 3-form field $H_{m_{1} m_{2} m_{3}}$ keeping only the scalar fields, supersymmetric branes are described by calibrated surfaces $[65,66,67]$. Calibrated surfaces are minimal surfaces within a given homology class. They are specified by supersymmetry. The mathematical theory of these objects has a long history and one can find an enlightening collection of the corresponding results in $[68,69]$.

The first convincing application of these ideas to physics are in the context of $N=2$ supersymmetric gauge theories in 4 dimensions which make use of $S U(2)$ Special Lagrangian (SLAG) calibration (supersymmetric 2-cycles) [2]. One expects for $N=1$ gauge theories an analogue behaviour. To be more precise their nonperturbative informations must be associated with $S U(3)$-SLAG submanifolds (supersymmetric 3-cycles), the case we will be concerned with in chapter 4 . However, unlike the supersymmetric 2-cycles, the geometry of supersymmetric 3-cycles and all higher odd dimensional SLAG cycles are not as transparent as in the case of $S U(2)-\mathrm{SLAG}$. Nevertheless concrete applications require a simple handling of such M5-brane solutions. Unfortunately for the $S U$ (3)-SLAG only extremely special solutions could be written down so far. Therefore we are interested in constructions which connect SLAG geometries of different dimensions to each other. This is the focus of this chapter.

We start to explain all the necessary notions in section 3.1 , which are required later on. 
Then in section 3.2 we will pick up an old idea, which allows to connect Lagrangian submanifolds of different dimensions to each other. After restriction to the class of SLAG submanifolds this construction fails since inevitably it generates a smoothly varying phase $\alpha$ on the projected cycles which prevents it to be SLAG ( $\alpha=$ const) again.

In section 3.3 the BPS equation (1.13) in the presence of a 3 -form $H$-field will be investigated for the possibility to reestablish a BPS property of projected cycles. The interesting point which comes out is the appearance of additional terms generated by the $H$-field with the capability to modify the differential equations of the SLAG conditions in the right way, while breaking further supersymmetries.

We will derive the modified differential equations ${ }^{*_{1}}$ and discuss their geometrical meaning.

\subsection{The SU(n)-SLAG Calibrations}

We start from the configurations of intersecting M5-branes in the two tables below (a bar denotes a negative sign of the eigenvalues of supersymmetries preserved by the brane). Here the notion "intersecting brane" should be taken as a more symbolic one. It is only comfortable to encode the information about the preserved supersymmetries in this manner.

\begin{tabular}{|l|lllllll|}
\hline & 1 & 2 & & & & $1 '$ & 2 \\
\hline \hline M5 & 1 & 2 & 3 & 4 & 5 & & \\
$\bar{M} 5$ & & & 3 & 4 & 5 & 6 & 7 \\
& & & & & & & \\
\hline
\end{tabular}

Tab. 3.1: $S U(2)-S L A G$

\begin{tabular}{|l|llllllll|}
\hline & 1 & 2 & 3 & & & 1 & 2 & 3 \\
\hline \hline M5 & 1 & 2 & 3 & 4 & 5 & & & \\
$\overline{\text { M5 }}$ & & & 3 & 4 & 5 & 6 & 7 & \\
M5 & & 2 & & 4 & 5 & 6 & & 8 \\
\hline
\end{tabular}

Tab. 3.2: $S U(3)-S L A G$

So the generalisation of the SUSY conditions of eq. (1.10) for the configuration depicted in Tab. 3.1 leads to the two equations below, one for each brane:

$$
\Gamma^{012345} \epsilon=\epsilon, \quad \Gamma^{034567} \epsilon=-\epsilon
$$

Using the split of 11d gamma matrices of eq. (1.12) this can be written in the form $\gamma^{i j} \epsilon=-\gamma^{i j} \epsilon$, which is an identity of gamma matrices applied to a spinor of preserved supersymmetries. The details can be easily filled in from the formulas in appendix A). The topology of a $S U(n)$-SLAG calibrated M5-brane is $\mathbb{R}^{1,5-n} \times M_{n}$ with $M_{n}$ the $S U(n)$ SLAG submanifold. In the absence of the self dual 3-form $h_{a b c}$ these projectors imply

\footnotetext{
${ }^{* 1}$ The authors of [70] considered the same questions independently.
} 
the SLAG differential equations as shown in [67]. There the authors analysed the first three terms of eq. (1.13) and reobtained the differential equations of the $S U(n)$-SLAG geometries, which for $S U(2)-S L A G$ and $S U(3)-S L A G$ read:

$$
\hat{\delta} \Theta_{\beta}{ }^{j}=\frac{-1}{2 \operatorname{det} e} \epsilon^{\alpha i}\{\frac{1}{2} \underbrace{\left[\partial_{a} X_{c}-\partial_{c} X_{a}\right]}_{\text {generic }} \gamma^{a} \gamma^{\prime c}+[\sum_{a} \partial_{a} X_{a}-\underbrace{\operatorname{det}(\partial X)}_{0 \text { for } S U(2)}] \gamma^{1} \gamma^{\prime 1}\}
$$

The term in front of $\gamma^{a} \gamma^{\prime c}$ enforces the M5-brane to be a Lagrangian submanifold, i.e. a submanifold of half of the dimension of the embedding space on which the symplectic forms restricts to zero. This can be seen by computing the pullback of the symplectic form under the embedding map. The symplectic form is defined by $\omega\left(X_{\mathbb{R}}, Y_{\mathbb{R}}\right)=g\left(J X_{\mathbb{R}}, Y_{\mathbb{R}}\right)$ with $J$ the complex structure and $g$ the Euclidean scalar product of the embedding space. Using the complex structure one can make a real vector $X_{\mathbb{R}}$ into a complex one $X_{\mathbb{C}}$ and the identity,

$$
<X_{\mathbb{C}}, Y_{\mathbb{C}}>_{\mathbb{C}}=g\left(X_{\mathbb{R}}, Y_{\mathbb{R}}\right)+i \cdot \underbrace{\omega_{\mathbb{R}}\left(X_{\mathbb{R}}, Y_{\mathbb{R}}\right)}_{0},
$$

states, that orthogonality in the real sense implies orthogonality in the complex sense and vice versa. Thus for a manifold to be Lagrangian the complexified tangent vectors must be complex orthonormalised. Locally the evaluation of the form

$$
\Phi=\left(\tilde{e}_{1}+i \tilde{e}_{2}\right) \wedge \ldots \wedge\left(\tilde{e}_{2 n-1}+i \tilde{e}_{2 n}\right)
$$

on the tangent planes ( $\tilde{e}_{i}$ a dual basis of $\left.e_{i}, J e_{i}=e_{i+1}\right)$ measures the phase $e^{i \alpha}$ of the unitary matrix build out of these unitary tangent vectors. In a spacetime frame the role of $\Phi$ is played by the holomorphic volume form $\Omega=d z^{1} \wedge \ldots \wedge d z^{n}$ living on the embedding space $X_{\mathbb{C}}$ regarded as a complex space and one obtains

$$
e^{i \alpha} \sqrt{g} d q^{1} \wedge \ldots \wedge d q^{n}=f^{*} \Omega=f^{*} \Re \mathfrak{e} \Omega+i \cdot f^{*} \Im \mathfrak{m} \Omega
$$

from which one can compute the $\tan \alpha$ as:

$$
\tan \alpha=\frac{\left[f^{*} \Im \mathfrak{m} \Omega\right]}{\left[f^{*} \Re \mathfrak{e} \Omega\right]}
$$

For the definition of the brackets see around eq. (3.12). The second term in formula (3.1) can be interpreted as the requirement $f^{*} \Im \mathrm{m} \Omega=0$, which by eq. (3.3) leads to $e^{i \alpha}=1$. In fact these are conditions referring only to the tangent planes of the submanifold they belong to. The reason for the simplicity of the $S U(2)-S L A G$ case is due to the simple structure of the space of 2-planes in $\mathbb{R}^{4}$, which satisfy further geometrical requirements as discussed in details in appendix C.3. It can be analysed by studying the associated Gauß map. The results can be summarised as:

- The space of 2-planes in $\mathbb{R}^{4}$ is isomorphic to $S_{+}^{2} \times S_{-}^{2}$. 
- Each point in $S_{+}^{2}$ can be identified with a complex structure of $\mathbb{R}^{4}$.

- In a fixed complex structure the intersection of $S_{+}^{2}$ with the orthogonal complement of the direction defined by the complex structure is a $S^{1}$. It corresponds to the $S^{1}$ of phases a Lagrangian plane can have.

The origin for the complications arising for $S U(3)-S L A G$ can be traced back to the complicated structure of the corresponding Gauß map.

\subsection{A Generic Property of Lagrangian Submanifolds}

A short calculation indicates a possible way out. Starting from a SLAG 3-cycle and only reshuffling terms in eq. (3.1) one finds:

$$
\begin{aligned}
\hat{\delta} \Theta_{\beta}^{j}=- & \frac{1}{2} \operatorname{det}\left(e^{-1}\right) \epsilon^{\alpha i}\left\{\frac{1}{2}\left[\partial_{a} X_{c}-\partial_{c} X_{a}\right] \gamma^{a} \gamma^{\prime c}+\left[\sum_{a} \partial_{a} X_{a}-\operatorname{det}(\partial X)\right] \gamma^{1} \gamma^{\prime 1}\right\} \\
= & -\frac{1}{2} \operatorname{det}\left(e^{-1}\right) \epsilon^{\alpha i}\left\{\frac{1}{2}\left[\partial_{1} X_{2}-\partial_{2} X_{1}\right] \gamma^{1} \gamma^{\prime 2}\right. \\
& +\left[\sum_{a} \partial_{a} X_{a}+\left(1-\partial_{1} X_{1} \partial_{2} X_{2}+\partial_{2} X_{1} \partial_{1} X_{2}\right) \partial_{3} X_{3}\right] \gamma^{1} \gamma^{\prime 1} \\
& +\left[\partial_{2} X_{3}\left(\partial_{1} X_{1} \partial_{3} X_{2}-\partial_{1} X_{2} \partial_{3} X_{1}\right)-\partial_{1} X_{3}\left(\partial_{1} X_{2} \partial_{3} X_{2}-\partial_{3} X_{1} \partial_{2} X_{2}\right)\right] \gamma^{1} \gamma^{\prime 1} \\
& \left.+\frac{1}{2}\left[\partial_{1} X_{3}-\partial_{3} X_{1}\right] \gamma^{1} \gamma^{\prime 3}+\frac{1}{2}\left[\partial_{2} X_{3}-\partial_{3} X_{2}\right] \gamma^{2} \gamma^{\prime 3}\right\}
\end{aligned}
$$

If one would forget about the dependence on the coordinate $q_{3}$ and in addition treats the transversal coordinate $X_{3}$ only as a certain function appearing in the differential equations one would project onto a Lagrangian submanifold of lower dimension but now with a non constant phase $\alpha$.

This idea could be made very precise since it is a well established property of Lagrangian submanifolds (see e.g. [71]). The basic ingredient is a Lagrangian submanifold $\Lambda_{X}$ of the symplectic manifold $X$ and a Lagrangian submanifold $\Lambda_{X \times Y}$ of the Cartesian product $X \times Y$ of $X$ with another symplectic manifold $Y$. We are interested in constructing a Lagrangian submanifold of $Y$ out of these data. Thereby the Cartesian product is made into a symplectic manifold by defining the symplectic form to be the sum of the symplectic forms on the individual constituents pulled back under the projection maps to $X$ and $Y$ respectively:

$$
\omega_{X \times Y}=\pi_{X}^{*} \omega_{X}+\pi_{Y}^{*} \omega_{Y}
$$


The main idea of the construction are depicted in Fig. 3.1 and will be explained soon. Our candidate $\lambda_{Y}$ for a Lagrangian submanifold $\Lambda_{Y}$ in $Y$

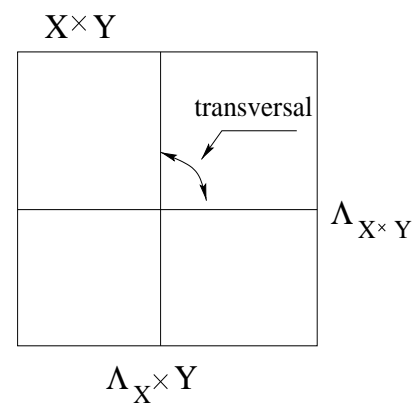

Fig. 3.1: The basic construction would be the projection into $Y$ of the (transversal) intersection below:

$$
\lambda_{Y}=\pi_{Y}\left(\Lambda_{X} \times Y \cap \Lambda_{X \times Y}\right)
$$

Let us start to analyse the properties of this intersection. What is its dimension? Of course we can reduce the computation of the dimension to the computation of the dimension of the intersecting tangent planes.

But then we can use a very simple formula for the dimension of the intersection of two subspaces of a vector space, say $V$. For $U_{1}, U_{2} \in V$

$$
\operatorname{dim}\left(U_{1} \cap U_{2}\right)=\operatorname{dim} U_{1}+\operatorname{dim} U_{2}-\operatorname{dim}\left(U_{1}+U_{2}\right)
$$

which can be checked by simply complete a basis of $U_{1} \cap U_{2}$ to a basis of $U_{1}$ or $U_{2}$, respectively. Choosing $U_{1}=T\left(\Lambda_{X} \times Y\right), U_{2}=T \Lambda_{X \times Y}$ and $V=U_{1}+U_{2}=T(X \times Y)$ (transversality!) we obtain the dimension of the intersection

$$
\begin{aligned}
\operatorname{dim}\left(\Lambda_{X} \times Y \cap \Lambda_{X \times Y}\right)= & \operatorname{dim} U_{1}+\operatorname{dim} U_{2}-\operatorname{dim}\left(U_{1}+U_{2}\right) \\
= & \left(\frac{1}{2} \operatorname{dim} X+\operatorname{dim} Y\right)+\frac{1}{2}(\operatorname{dim} X+\operatorname{dim} Y) \\
& -(\operatorname{dim} X+\operatorname{dim} Y) \\
= & \frac{1}{2} \operatorname{dim} Y
\end{aligned}
$$

Now we investigate the behaviour of the intersection under the projection map to $Y$. Especially interesting is a possible loss of dimensionality under this projection. Therefore we want to know which are the tangent vectors of the intersection which get mapped to zero in the image of the projection. Since the preimage do have the right dimension of a Lagrangian submanifold of $Y$, we would like to guarantee that there is no tangent vector of the intersection which get mapped to zero in the image beside the zero vector, i.e. the projection map is an immersion. This is the idea behind the following computation. To analyse this question we restrict ourself to the first member $\Lambda_{X} \times Y$ of the intersection. Let $i$ denote its immersion into $X \times Y$. The pullback of the symplectic form $\omega_{X \times Y}$ of the Cartesian product onto $\Lambda_{X} \times Y$ under the immersion $i$ yields

$$
i^{*} \omega_{X \times Y}=\underbrace{i^{*} \pi_{X}^{*} \omega_{X}}_{0 !}+i^{*} \pi_{Y}^{*} \omega_{Y}
$$

One terms drops out since we pulled back the symplectic form of $X$ to a Lagrangian submanifold $\Lambda_{X}$ of $X$. Now we choose a tangent vector of the intersection which is 
mapped to zero under the projection map:

$$
d \pi_{Y}(\xi)=0
$$

Choose $\tilde{\xi} \in T\left(\Lambda_{X} \times Y\right)$ then the following equality holds

$$
i^{*} \omega_{X \times Y}(\xi, \tilde{\xi})=i^{*} \pi_{Y}^{*} \omega_{Y}(\xi, \tilde{\xi})=i^{*} \omega_{Y}\left(d \pi_{Y}(\xi), \tilde{\xi}\right)=0
$$

by the equation above. On the other side if we evaluate $\omega_{X \times Y}$ on $\xi$ and $\tilde{\xi} \in T\left(\Lambda_{X \times Y}\right)$ the answer is zero by definition. Since the intersection is transversal $\omega_{X \times Y}(\xi, \circ) \equiv 0$. But by the nondegeneracy of the symplectic form this implies $\xi=0$. Therefore the projection is immersiv. Last but not least the symplectic form reduces to zero on the intersection since it is contained in a Lagrangian submanifold. All in all we end up with the result that $\lambda_{Y}$ is a Lagrangian submanifold of $Y$.

\subsection{Modified BPS Equations}

After presenting the basic idea we want to collect evidence for the possibility to implement the above mentioned construction on the level of BPS solutions. By what is known about BPS solutions so far there is no doubt about the necessity to take the self dual 3-form field $h_{m_{1} m_{2} m_{3}}$ into account to get a chance that these new BPS solution exists. After inclusion of this field the general SUSY variation for a M5-brane in

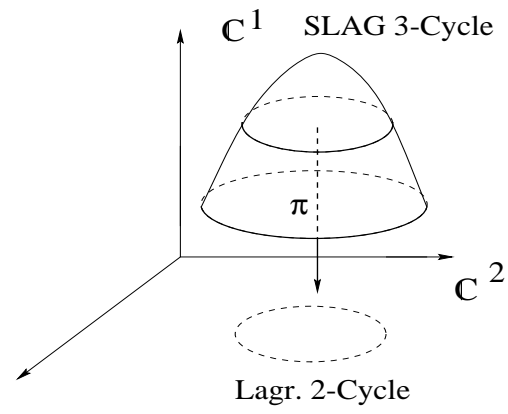

Fig. 3.2: The projection $\pi_{Y}$ a flat background are given in eq. (1.13). The new ingredients are the additional terms, which appear after turning on $h_{m_{1} m_{2} m_{3}}$. To uncover the structure of these terms we consider the contribution of the 4th and 5th term of eq. (1.13) to the BPS equations. These terms contain the full dependence on the field $h_{m_{1} m_{2} m_{3}}$. Since $h_{m_{1} m_{2} m_{3}}$ is self dual ${ }^{*_{2}}$,

$$
h^{m_{1} m_{2} m_{3}}=\frac{1}{3 !} \frac{1}{\sqrt{-g}} \epsilon^{m_{1} \ldots m_{6}} h_{m_{4} m_{5} m_{6}},
$$

the purely space like components can be computed from $h_{0 m n}$, i.e.

$$
h^{m_{1} m_{2} m_{3}}=\frac{1}{2} \frac{1}{\sqrt{-g}} \underbrace{\epsilon^{m_{1} m_{2} m_{3} 0 m n}}_{\text {Minkowskian }} h_{0 m n}=-\frac{1}{2} \frac{1}{\sqrt{-g}} \underbrace{\epsilon^{m_{1} m_{2} m_{3} m n}}_{\text {Euclidean }} h_{0 m n}
$$

${ }^{*}$ For the conventions see appendix A. 
This motivates the introduction of the following abbreviations

$$
\begin{aligned}
F_{m n} & =h_{0 m n} \in \Lambda^{2}\left(\mathbb{R}^{5}\right), \\
(* F)^{r s t} & =\frac{1}{2} \frac{1}{\sqrt{-g}} \epsilon^{r s t m n} h_{0 m n} \in \Lambda^{3}\left(\mathbb{R}^{5}\right) .
\end{aligned}
$$

with $\epsilon^{\text {rstmn }}$ the five dimensional Euclidean $\epsilon$-symbol. With these definitions the fourth term can be rewritten like:

$$
\begin{aligned}
\text { thh } & =-h^{m_{1} m_{2} m_{3}} \partial_{m_{2}} X_{c_{2}} \partial_{m_{3}} X_{c_{3}}\left(\gamma_{m_{1}}\right)_{\alpha \beta}\left(\gamma^{\prime c_{2} c_{3}}\right)_{i}{ }^{j} \\
& =-h^{0 m n} \partial_{m} X_{c_{2}} \partial_{n} X_{c_{3}}\left(\gamma_{0}\right)_{\alpha \beta}\left(\gamma^{\prime c_{2} c_{3}}\right)_{i}{ }^{j}-h^{p m n} \partial_{m} X_{c_{2}} \partial_{n} X_{c_{3}}\left(\gamma_{p}\right)_{\alpha \beta}\left(\gamma^{\prime c_{2} c_{3}}\right)_{i}{ }^{j} \\
& =F^{m n} \partial_{m} X_{c_{2}} \partial_{n} X_{c_{3}} \gamma_{0} \gamma^{\prime c_{2} c_{3}}+(* F)^{m n p} \partial_{m} X_{c_{2}} \partial_{n} X_{c_{3}} \gamma_{p} \gamma^{\prime c_{2} c_{3}},
\end{aligned}
$$

and the last one simplifies according to

$$
\begin{aligned}
5 \text { th } & =-\frac{1}{3} h^{m_{1} m_{2} m_{3}}\left(\gamma_{m_{1} m_{2} m_{3}}\right)_{\alpha \beta} \\
& =-h^{0 m n} \gamma_{0 m n}-\frac{1}{3} h^{m n p} \gamma_{m n p} \\
& =-h^{0 a b} \gamma_{0 a b}-\frac{1}{3} \frac{1}{3 !} \frac{1}{\sqrt{-g}} \epsilon^{m n p r s t} h_{r s t} \frac{1}{3 !} \epsilon_{m n p u v w} \gamma^{u v w} \\
& =-h^{0 m n} \gamma_{0 m n}+\frac{1}{\sqrt{-g}} \frac{1}{12} \delta_{0 v w m n n}^{0 s t m n} h_{0 s t} \gamma^{0 v w} \\
& =-h^{0 m n} \gamma_{0 m n}+\frac{1}{\sqrt{-g}} \frac{1}{2} \delta_{0 v w}^{0 s t} h_{0 s t} \gamma^{0 v w} \\
& =-h^{0 m n} \gamma_{0 m n}+\frac{1}{\sqrt{-g}} \frac{1}{2} 2 h_{0 m n} \gamma^{0 m n} \\
& =F^{m n} \gamma_{0 m n}+\frac{1}{\sqrt{-g}} F_{m n} \gamma^{0 m n}
\end{aligned}
$$

Then the BPS equation is modified by the following expression:

$$
\begin{gathered}
\hat{\delta} \Theta_{\beta}{ }^{j}=\ldots-\frac{1}{2} \epsilon^{\alpha i}\left\{F^{m n} \partial_{m} X_{c_{2}} \partial_{n} X_{c_{3}} \gamma_{0} \gamma^{\prime c_{2} c_{3}}+(* F)^{m n p} \partial_{m} X_{c_{2}} \partial_{n} X_{c_{3}} \gamma_{p} \gamma^{\prime c_{2} c_{3}}\right. \\
\left.+F^{m n} \gamma_{0 m n}+\frac{1}{\sqrt{-g}} F_{m n} \gamma^{0 m n}\right\}
\end{gathered}
$$

If one does not introduce additional projectors, which further reduce the amount of supersymmetry, the last equation states that the cycle remains $S U(n)$-SLAG but will be deformed by the field $h_{m_{1} m_{2} m_{3}}$. Technically speaking the moduli of the cycle become functions of the field $h_{m_{1} m_{2} m_{3}}$ and are partially eliminated in favour of the degrees of freedom of the field $h_{m_{1} m_{2} m_{3}}$. One the other hand breaking SUSY further by additional projectors the $S U(n)$-SLAG conditions will be affected as we will discuss now. 


\subsubsection{Investigation of the Basic Example}

Now we specialise all considerations for the $S U(2)$-SLAG example as given in Tab. 3.1 which contains the generic behaviour. One can use a duality relation of the chiral $6 \mathrm{~d}$ gamma matrices (see eq. (A.1) in the appendix) to further simplify eq. (3.5). We want to put as many as possible $\gamma$-terms into the form $\gamma_{0} \gamma^{\prime c_{1} c_{2}}$. Writing out the corresponding expression for the 2-cycle one obtains

$$
(* F)^{m n p} \partial_{m} X_{c_{2}} \partial_{n} X_{c_{3}} \gamma_{p} \gamma^{\prime c_{2} c_{3}}=2(* F)^{12 p} \partial_{1} X_{[1} \partial_{2} X_{2]} \gamma_{p} \gamma^{\prime 12}
$$

This has to be discussed for $c=3, \ldots, 5$. With the help of the projectors of Tab. 3.1 and the $6 \mathrm{~d}$ duality relation eq. (A.1) we may compute,

$$
\gamma_{3} \gamma^{12} \epsilon=-\gamma_{0} \gamma^{12} \epsilon
$$

and $(* F)^{123}=\frac{1}{\sqrt{-g}} F_{45}$. Similarly we may conclude $\gamma_{045}=-\gamma_{0} \gamma^{\prime 12}$. Then the BPS equations read

$$
\begin{aligned}
\hat{\delta} \Theta_{\beta}{ }^{j}=- & \frac{1}{2} \operatorname{det}\left(e^{-1}\right) \epsilon^{\alpha i}\left\{\frac{1}{2}\left[\partial_{a} X_{c}-\partial_{c} X_{a}\right] \gamma^{a} \gamma^{\prime c}+\left[\sum_{a} \partial_{a} X_{a}\right] \gamma^{1} \gamma^{\prime 1}\right\} \\
-\frac{1}{2} \epsilon^{\alpha i} & \left\{2\left(F^{12}-\frac{1}{\sqrt{-g}} F_{45}\right)\left(\partial_{1} X_{1} \partial_{2} X_{2}-\partial_{2} X_{1} \partial_{1} X_{2}\right)\right. \\
& \left.-2\left(F^{12}+F^{45}\right)+2 \frac{1}{\sqrt{-g}}\left(F_{12}+F_{45}\right)\right\} \gamma_{0} \gamma^{\prime 2}+\ldots
\end{aligned}
$$

where the dots are the terms for $c=4,5$ implying that that the corresponding components of $F^{m n}$ vanish. The vielbein $e^{a}{ }_{\mu}$ (see appendix A) has a very simple form:

$$
e^{a}{ }_{\mu}=\left(\begin{array}{cccc}
1 & 0 & 0 & \mathbf{0} \\
0 & e^{1}{ }_{1} & e^{1}{ }_{2} & \mathbf{0} \\
0 & e^{2}{ }_{1} & e^{2}{ }_{2} & \mathbf{0} \\
0 & 0 & 0 & \mathbb{1}
\end{array}\right)
$$

The transformation to the tangent frame is performed by $F^{m n}=\tilde{e}_{a}^{m} \tilde{e}_{b}^{n} F^{a b}$ and yields

$$
F^{12}=\tilde{e}_{a}^{1} \tilde{e}^{2}{ }_{b} F^{a b}=\frac{1}{\sqrt{-g}} F^{12} \quad F^{45}=\tilde{e}^{4}{ }_{a} \tilde{e}^{5}{ }_{b} F^{a b}=F^{45} .
$$

Since in the tangent frame $F^{12}=F_{12}$ and the same valid for $F^{45}$ eq. (3.6) reads:

$$
\begin{aligned}
\hat{\delta} \Theta_{\beta}{ }^{j}= & -\frac{1}{2} \operatorname{det}\left(e^{-1}\right) \epsilon^{\alpha i}\left\{\frac{1}{2}\left[\partial_{a} X_{c}-\partial_{c} X_{a}\right] \gamma^{a} \gamma^{\prime c}+\left[\sum_{a} \partial_{a} X_{a}\right] \gamma^{1} \gamma^{\prime 1}\right. \\
& \left.+\left[2\left(F_{12}-F_{45}\right)\left(\partial_{1} X_{1} \partial_{2} X_{2}-\partial_{2} X_{1} \partial_{1} X_{2}\right)-2\left(F_{12}-F_{45}\right)+2 \sqrt{-g}\left(F_{12}-F_{45}\right)\right] \gamma_{0} \gamma^{\prime 12}\right\} \\
& +\ldots
\end{aligned}
$$


The new term is precisely (for the notation see around eq. (3.12))

$$
2\left(F_{12}-F_{45}\right)\left(\sqrt{-g}-\left[f^{*} \Re \mathfrak{e} \Omega\right]\right)
$$

which for a $S U(2)$-SLAG 2-cycle already vanishes, as a consequence of eq. (3.2) and $f^{*} \Im \mathfrak{m} \Omega=0$. Thus the picture does not change. A completely different behaviour arises after breaking further supersymmetries. Concretely we will break supersymmetry by imposing additional projectors which modify either the Lagrangian or the constant phase condition. The latter can be translated to the altered brane picture below [26]:

\begin{tabular}{|l|lllllll|}
\hline & 1 & 2 & & & & $1^{\prime}$ & 2 \\
\hline \hline M5 & 1 & 2 & 3 & 4 & 5 & & \\
$\overline{\mathrm{M} 5}$ & & & 3 & 4 & 5 & 6 & 7 \\
M2 & 1 & & & & & & 7 \\
\hline
\end{tabular}

Tab. 3.3: Brane configuration

From the additional " $M 2$-brane projector" $\Gamma_{017} \epsilon=\eta \epsilon\left(\Gamma_{a} 11 \mathrm{~d}\right.$ tangent frame gamma matrices, $\eta$ a sign) one obtains

$$
\Gamma_{067} \epsilon=-\eta \Gamma_{16} \epsilon
$$

and by the construction of $\Gamma_{a}$ (see eq. (1.12)) this is identical to

$$
\gamma_{0} \gamma^{12} \epsilon=\eta \gamma^{1} \gamma^{11} \epsilon
$$

This equality generates an inflow from the purely $h$-field terms to the term proportional to $\gamma^{1} \gamma^{11}$ and modifies the SLAG condition while preserving the Lagrangian property ${ }^{*_{3}}$. If one writes out the resulting BPS equations, one finds:

$$
\begin{aligned}
\hat{\delta} \Theta_{\beta}{ }^{j}= & -\frac{1}{2} \operatorname{det}\left(e^{-1}\right) \epsilon^{\alpha i}\left\{\frac{1}{2}\left[\partial_{1} X_{2}-\partial_{2} X_{1}\right] \gamma^{1} \gamma^{\prime 2}+\left[\sum_{a} \partial_{a} X_{a}\right] \gamma^{1} \gamma^{\prime 1}\right. \\
& +2\left(F_{12}-F_{45}\right)\left(\sqrt{-g}-\left[f^{*} \Re \mathfrak{e} \Omega\right]\right) \underbrace{\gamma_{0} \gamma^{\prime 12}}_{\eta \gamma^{1} \gamma^{\prime 1}}\}
\end{aligned}
$$

We can solve for the $F_{a b}$ :

$$
F_{12}-F_{45}=-\frac{\eta}{2} \frac{\left[f^{*} \Im \mathfrak{m} \Omega\right]}{\sqrt{-g}-\left[f^{*} \Re \mathfrak{e} \Omega\right]}
$$

\footnotetext{
${ }^{*}$ Instead of including the $M 2$-brane projector $\Gamma_{017} \epsilon=\eta \epsilon$ one could choose $\Gamma_{016} \epsilon=\kappa \epsilon$, which modifies the Lagrangian condition but preserves the other equation. But this case is not so well suited for our purposes but seems to be the generic one for higher dimensions.
} 
Before we discuss the consequence of this choice let us remind of some of the geometrical formulas. The pullbacks of the embedding space differential forms $\omega, \Re \mathfrak{e} \Omega$ and $\Im \mathfrak{m} \Omega$ under the map $f$ read

$$
\begin{aligned}
f^{*} \omega & =\left(\partial_{2} X_{1}-\partial_{1} X_{2}\right) d q^{1} \wedge d q^{1} \\
f^{*} \Re \mathfrak{e} \Omega & =\left(1-\partial_{1} X_{1} \partial_{2} X_{2}+\partial_{2} X_{1} \partial_{1} X_{2}\right) d q^{1} \wedge d q^{2} \\
f^{*} \Im \mathfrak{m} \Omega & =\left(\partial_{1} X_{1}+\partial_{2} X_{2}\right) d q^{1} \wedge d q^{2} .
\end{aligned}
$$

We will need the prefactors in front of the differentials which we denote by $\left[f^{*} \omega\right]$ and so on. By slight abuse of language we will denote the induced metric on the embedded submanifold $M^{2}$ with the same name $g_{i j}$ as for the full M5-brane. This is justified by the fact that the remaining components of the M5-brane metric are \pm 1 and one has only to keep the correct sign for the determinant in mind. The induced metric is given by

$$
g_{i j}=\left(\begin{array}{cc}
1+\left(\partial_{1} X_{1}\right)^{2}+\left(\partial_{1} X_{2}\right)^{2} & \partial_{1} X_{1} \partial_{2} X_{1}+\partial_{1} X_{2} \partial_{2} X_{2} \\
\partial_{1} X_{1} \partial_{2} X_{1}+\partial_{1} X_{2} \partial_{2} X_{2} & 1+\left(\partial_{2} X_{1}\right)^{2}+\left(\partial_{2} X_{2}\right)^{2}
\end{array}\right)
$$

and the following algebraic identity (see appendix C.2)

$$
\operatorname{det} g=\left[f^{*} \Im \mathfrak{m} \Omega\right]^{2}+\left[f^{*} \Re \mathfrak{e} \Omega\right]^{2}+\left[f^{*} \omega\right]^{2}
$$

holds. Combining this identity with the BPS equation $f^{*} \omega=0$ and the BPS equation (3.11) one can solve for $\left[f^{*} \Im \mathfrak{m} \Omega\right]$ in terms of $\left[f^{*} \Re_{\mathfrak{e}} \Omega\right]$. There are two solutions. The first one is simply $\left[f^{*} \Im \mathrm{m} \Omega\right]=0$ again. The second one can be rewritten into an expression for the phase (see eq. (3.3)),

$$
\tan \alpha=-\eta \frac{4\left(F_{12}-F_{45}\right)}{4\left(F_{12}-F_{45}\right)^{2}-1}
$$

which is generically not constant. Of course one has to ensure that the equation of motion for the spacetime 3 -form is satisfied. The simplest way this can be ensured is to take the tangent frame components $F_{12}$ and $F_{45}$ to be constant. As can be seen from eq. (3.8) the corresponding spacetime components are non constant. Nevertheless in this situation the cycle is not anymore SLAG but still a Lagrangian submanifold.

As can be seen from the Gauß map (see appendix C.3) the parameter $F_{12}-F_{45}$ can be identified with one of the angles parametrising the sphere $S_{+}^{2}$ of complex structures on $\mathbb{R}^{4}$. Completely analogous for the other choice of the projector (see footnote on page 49) one can identify the parameter with the second angle.

If $F_{12}=F_{45}$ eq. (3.10) vanishes. The closure of $H$ leads to $F_{12}=F_{45} \stackrel{!}{=} 0$ and one gets back the $S U(2)$-SLAG solution, with an spontaneously enhanced supersymmetry. 


\subsubsection{The 3-Cycle Case}

For the 3-cycle an unexpected behaviour appears. As will become clear, soon, we do not have the freedom to modify either the phase or Lagrangian condition independently but there is only the possibility to change the latter one. We start from the most general pattern of M5-branes and M2-branes, which preserve a common supersymmetry in Tab. 3.4.

\begin{tabular}{|l|lllllllll|}
\hline & & 1 & 2 & 3 & & & $1^{\prime}$ & $2^{\prime}$ & 3 \\
\hline \hline M5 & 0 & 1 & 2 & 3 & 4 & 5 & & & \\
$\overline{\text { M5 }}$ & 0 & & & 3 & 4 & 5 & 6 & 7 & \\
M5 & 0 & & 2 & & 4 & 5 & 6 & & 8 \\
M2 & 0 & & & 3 & & & & & 8 \\
\hline M2 & 0 & 1 & & & & & 6 & & \\
M2 & 0 & & 2 & & & & & 7 & \\
$\overline{\mathrm{M} 5}$ & 0 & 1 & & & 4 & 5 & & & 8 \\
\hline
\end{tabular}

Tab. 3.4: Brane configuration

The branes in the lower box are consequences of the projectors coming from the branes in the upper box and can therefore added for free.

Repeating the same steps already done in the last section one finds that the M2-brane projector can be rewritten like

$$
\begin{aligned}
\Gamma_{038} \epsilon & =\eta \epsilon \quad / \cdot \Gamma_{36} \\
\Gamma_{068} \epsilon & =-\eta \Gamma_{36} \epsilon \\
\gamma_{0} \gamma^{\prime 13} & =-\eta \gamma^{1} \gamma^{\prime 3} .
\end{aligned}
$$

From the last line of this formulas one recognises that the additional projector contribute to the Lagrangian conditions, now. Completely analogues the other two M2-branes generate contributions to the same class of terms:

$$
\begin{aligned}
& \gamma_{0} \gamma^{\prime 12}=-\eta \gamma^{1} \gamma^{\prime 2} \\
& \gamma_{0} \gamma^{\prime 23}=-\eta \gamma^{2} \gamma^{\prime 3}
\end{aligned}
$$

After taking notice of this effect we have to care about the possibility to find nontrivial solutions for the closed 3 -form $\tilde{H}_{m_{1} m_{2} m_{3}}$. Now we added a twiddle to $H$ if it is the spacetime form. Nevertheless let us begin the discussion with the 3 -form field $h_{a b c}$ in a 
tangent frame. The only non vanishing components are

$$
h_{012}=a, \quad h_{013}=b, \quad h_{023}=c .
$$

We can compute the tangent frame components of $H_{a b c}$ from eq. (1.11) and obtain

$$
\begin{array}{ll}
H_{012}=\frac{a}{4 a^{2}+4 b^{2}+4 c^{2}+1} & H_{345}=\frac{a}{4 a^{2}+4 b^{2}+4 c^{2}-1} \\
H_{013}=\frac{b}{4 a^{2}+4 b^{2}+4 c^{2}+1} & H_{245}=\frac{c}{4 a^{2}+4 b^{2}+4 c^{2}-1} \\
H_{023}=\frac{c}{4 a^{2}+4 b^{2}+4 c^{2}+1} & H_{145}=\frac{c}{4 a^{2}+4 b^{2}+4 c^{2}-1}
\end{array}
$$

Last but not least the spacetime components can be computed via the vielbein $e^{a}{ }_{m}$ (see appendix A) which is given by:

$$
e_{m}^{a}=\left(\begin{array}{ccccc}
1 & 0 & 0 & 0 & 0 \\
0 & \sqrt{g_{11}} & \frac{g_{12}}{\sqrt{g_{11}}} & \frac{g_{13}}{\sqrt{g_{11}}} & 0 \\
0 & 0 & \sqrt{\frac{\left|g_{11} g_{22}-g_{12}^{2}\right|}{g_{11}}} & \frac{g_{11} g_{23}-g_{133} g_{12}}{g_{11} g_{22}-g_{12}^{2}} \sqrt{\frac{\left|g_{11} g_{22}-g_{12}^{2}\right|}{g_{11}}} & 0 \\
0 & 0 & 0 & \sqrt{\frac{g}{\left|g_{11} g_{22}-g_{12}^{2}\right|}} & 0 \\
0 & 0 & 0 & 0 & \mathbb{1}
\end{array}\right) .
$$

The spacetime components $\tilde{H}_{m_{1} m_{2} m_{3}}$ can be obtained from

$$
\tilde{H}_{m_{1} m_{2} m_{3}}=H_{a_{1} a_{2} a_{3}} e^{a_{1}}{ }_{m_{1}} e^{a_{2}} m_{2} e^{a_{3}} m_{3} .
$$

The only point to ensure is the closure of the spacetime 3 -form $\tilde{H}$, i.e.

$$
\begin{aligned}
d \tilde{H} & =d x^{4} \wedge d x^{5} \wedge\left[\left(\frac{\partial \tilde{H}_{245}}{\partial x_{1}}-\frac{\partial \tilde{H}_{145}}{\partial x_{2}}\right) d x^{1} \wedge d x^{2}+\left(\frac{\partial \tilde{H}_{345}}{\partial x_{1}}-\frac{\partial \tilde{H}_{145}}{\partial x_{3}}\right) d x^{1} \wedge d x^{3}\right. \\
& \left.+\left(\frac{\partial \tilde{H}_{345}}{\partial x_{2}}-\frac{\partial \tilde{H}_{245}}{\partial x_{3}}\right) d x^{2} \wedge d x^{3}\right]-\left[\frac{\partial \tilde{H}_{012}}{\partial x_{3}}-\frac{\partial \tilde{H}_{013}}{\partial x_{2}}+\frac{\partial \tilde{H}_{023}}{\partial x_{1}}\right] d x^{0} \wedge \ldots \wedge d x^{3}=0 .
\end{aligned}
$$

Since $d x^{0}$ appears only together with the second bracket, the closure implies two independent conditions on $\tilde{H}$. From the first bracket in eq. (3.14) it follows that

$$
\tilde{H}_{m 45}=\partial_{m} f\left(x_{1}, x_{2}, x_{3}\right)
$$


Solve for the tangent frame components $H_{145}, H_{245}$ and $H_{345}$

$$
H_{a 45}=e_{a}^{m} \tilde{H}_{m 45}=e_{a}{ }^{m} \partial_{m} f
$$

Introduce the abbreviation

$$
\|H\|^{2}=\delta^{a b} H_{a 45} H_{b 45}=\delta^{a b} e_{a}^{m} e_{b}^{n} \partial_{m} f \partial_{n} f=\partial^{m} f \partial_{m} f
$$

one computes from eq. (3.13) the parameters $a, b$ and $c$ :

$$
\begin{aligned}
a & =H_{345} \frac{1+\sqrt{1+16\|H\|^{2}}}{8\|H\|^{2}} \\
b & =-H_{245} \frac{1+\sqrt{1+16\|H\|^{2}}}{8\|H\|^{2}} \\
c & =H_{145} \frac{1+\sqrt{1+16\|H\|^{2}}}{8\|H\|^{2}}
\end{aligned}
$$

Then one can calculate from eq. (3.13) the tangent frame components

$$
H_{0 a b}=\epsilon_{a b c} \frac{H_{c 45}}{\sqrt{1+16\|H\|^{2}}}
$$

and finally spacetime components $\tilde{H}_{0 m n}$. Finally the function $f\left(x_{1}, x_{2}, x_{3}\right)$ is determined from inserting the last expression into the remaining differential equation in eq. (3.14), which follows from the closure of $\tilde{H}$.

Proof.

$$
\begin{aligned}
0=\epsilon^{m n p} \frac{\partial}{\partial x_{p}}\left[\tilde{H}_{0 m n}\right] & =\epsilon^{m n p} \frac{\partial}{\partial x_{p}}\left[H_{0 a b} e^{a}{ }_{m} e^{b}{ }_{n}\right] \\
& =\epsilon^{m n p} \frac{\partial}{\partial x_{p}}[\epsilon_{a b c} \underbrace{H_{c 45}}_{e^{c_{\alpha} \partial^{\alpha} f}} \frac{1}{\sqrt{1+16\|H\|^{2}}} e^{a}{ }_{m} e^{b}{ }_{n}] \\
& =\frac{\partial}{\partial x_{p}}\left[\sqrt{g} \partial^{p} f \frac{1}{\sqrt{1+16 \partial^{m} f \partial_{m} f}}\right]
\end{aligned}
$$

The final result can be put in a more significant form imitating the structure of the usual Laplace equation. Thus $f$ can be seen as a solution of the deformed Laplacian:

$$
\frac{1}{\sqrt{g}} \frac{\partial}{\partial x^{p}}\left[\sqrt{g} \partial^{p} f \frac{1}{\sqrt{1+16 \partial^{m} f \partial_{m} f}}\right]=0 .
$$




\subsection{Concluding Remarks}

We have shown that in the construction of supersymmetric solutions the field $h_{a b c}$ can be used to go beyond the case of standard calibrations without loosing a geometric picture. The physical three form field turns out as closely related to the complex structure. In two recent paper $[72,8]$ the author constructed the calibration form for the case at hand and demonstrated a method to estimate certain physical quantities by evaluating the forms on the cycle. Since it becomes clear here that the $H$-field gain a geometric interpretation, it should be possible to estimate a geometric quantity within this bound. This would allow to extend the notion of calibrated surfaces in a proper sense.

Furthermore the idea that the information of 3-cycles and 2-cycles are correlated was made more precise. The limitations of this calculation are mainly due to the restriction to a flat metric SUGRA background but this can be improved in principle. Partial results are available for non flat backgrounds [73, 74]. 


\section{Chapter 4}

\section{N=1 Gauge Theories \& 3-Cycles}

From the work of Witten [2], it was recognised that many interesting non-perturbative results of supersymmetric field theories can be derived from 11-dimensional M-theory. So it was shown that $N=2$ supersymmetric gauge theories can be solved via M-theory by lifting the corresponding 10-dimensional type IIA brane configurations [36] to 11 dimensions (see Tab. 4.1 and 4.2). As a result the intersection of $n$ parallel NS5-branes and $k$ suspended D4-branes is described in 11 dimensions by a complex curve, which is a supersymmetric 2-cycle embedded in the four dimensional space $\mathbb{R}^{3} \times S^{1}$. This curve precisely agrees with the famous Seiberg-Witten curves [75] of $N=2$ supersymmetric field theories.

\begin{tabular}{|l|lllllllll|}
\hline & 1 & 2 & 3 & 4 & 5 & 6 & 7 & 8 & 9 \\
\hline \hline NS5 & 1 & 2 & 3 & 4 & 5 & & & & \\
D4 & 1 & 2 & 3 & & & 6 & & & \\
\hline
\end{tabular}

Tab. 4.1: IIA

\begin{tabular}{|l|llllllllll|}
\hline & 1 & 2 & 3 & 4 & 5 & 6 & 7 & 8 & 9 & 10 \\
\hline \hline M5 & 1 & 2 & 3 & 4 & 5 & & & & & \\
M5 & 1 & 2 & 3 & & & 6 & & & & 10 \\
\hline
\end{tabular}

Tab. 4.2: M-Theory

The D4-branes exert a force on the NS5-branes causing them to bend. As explained in section 1.1.3 the tensions of the NS5-brane and the tensions of the D4-brane have a different behaviour in terms of the string coupling constant, $T_{5} \sim g_{s}^{-2}$ compared to $T_{4} \sim g_{s}^{-1}$, so classically the NS5-brane is much more heavier and there is no bending. Therefore bending is a quantum effect. As a result of bending, NS5-branes on which D4-branes end does not have a definite value of $x^{6}$. More specifically, when there is a bending which moves the two NS5-branes towards each other, the gauge coupling in eq. (4.2) becomes strong at high energies, i.e. we deal with an IR free theory. Conversely, if the bending is outwards, there is an asymptotically free theory. In four dimensions the bending is logarithmic with the distance $|v|$, whereas in $d$ dimensions the local bending of the NS5-branes goes like $|v|^{d-4}, v$ being the 'position' of the D4-brane on the world 
volume of the NS5-brane. This shows that in dimensions $d<4$ all gauge theories are asymptotically free, and all gauge theories with finite gauge coupling are IR free for $d>4$. The bending is absent if the number of left D4-branes ending on a given NS5brane is equal to the number of D4-branes ending from the right (see Fig. 4.1).

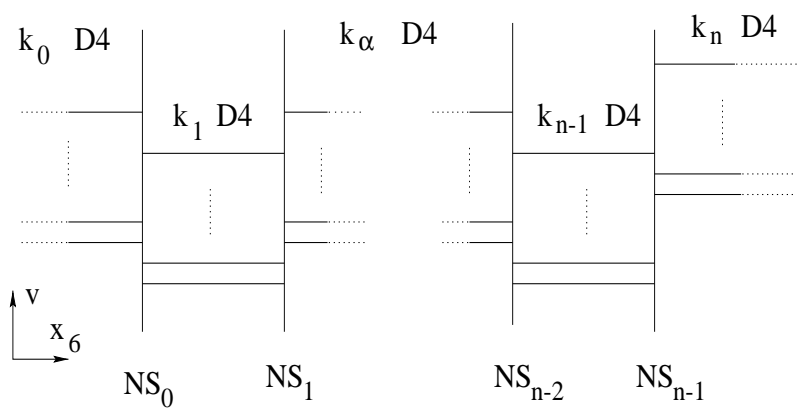

Fig. 4.1: The $\mathrm{N}=2$ brane configuration

In one-loop perturbation theory in four dimensions, the bending of the NS5-branes leads to a logarithmic variation of $x_{\alpha}^{6}$ in terms of $v$ :

$$
x_{\alpha}^{6}=\left(k_{\alpha}-k_{\alpha-1}\right) \log |v| .
$$

Taking into account that the square of the inverse gauge coupling is related to the distance of the NS5-branes, the logarithmic running of the gauge coupling $g_{\alpha}$ is determined as

$$
\frac{1}{g_{\alpha}^{2}}=\frac{-2 k_{\alpha}+k_{\alpha+1}+k_{\alpha-1}}{g_{s}} \log |v| .
$$

Note that the prefactor $b_{\alpha}^{N=2}=-2 k_{\alpha}+k_{\alpha+1}+k_{\alpha-1}$ precisely agrees with the $N=2 \beta$ function coefficient of the gauge group $S U\left(k_{\alpha}\right)$ with $N_{f}=k_{\alpha+1}+k_{\alpha-1}$ fundamental matter fields. In this way the shape of the branes incorporates the 1-loop effects in field theory. In $N=2$ field theory there are no higher loop effects. However there are still nonperturbative effects due to instantons. These instantons can be seen directly in the brane picture, namely the D0-branes are instantons within D4-branes. The problem is now to solve the theory by including all these effects. This can be done by "lifting" the IIA configuration to M-theory [2]. The advantage of considering the above configuration of branes in M-theory is that the D4-branes and NS5-branes descend from the same object, the M5-brane (see subsection 1.1.3). The intersection of the D4- and NS5-branes in IIA is singular but smoothed out in M-theory and in fact it is possible to consider all the D4-branes and NS5-branes as a single M5-brane with a nontrivial worldvolume. The conditions for preserving the equivalent of $\mathrm{N}=2$ supersymmetry in $\mathrm{d}=4$, i.e. 8 supercharges in 11 dimensions, restrict the embedding of the M5-brane worldvolume to 
be a $S U(2)-S L A G$ submanifold (see the discussion in chapter 3) and it is possible to find the function describing this embedding explicitly. The outcome of the analysis is that the M5-brane worldvolume is of the form $\mathbb{R}^{1,3} \times \Sigma^{(2)}$, with $\Sigma^{(2)}$ a complex Riemann surface holomorphically embedded into $\mathbb{R}^{3} \times S^{1}$. This curve incorporates the classical IIA brane set up as well as the field theory 1-loop corrections through the shape of the M5-brane. Finally the non-perturbative instantons are also automatically included since D0-branes are simply Kaluza-Klein momentum modes of compactified M-theory.

Why does this procedure work? The solution of the gauge theory is found by using the duality between 11d SUGRA and IIA string theory. When $R_{11}$ is large, 11d SUGRA is valid. On the other hand one identifies the gauge group and matter content at small $R_{11}$, where one works in weakly coupled IIA string theory and the analysis of [36] applies. In order to decouple the bulk modes from the gauge theory we have to take the string scale $m_{s}$ and the Planck scale $m_{p}$ to infinity and in order to decouple the KK modes from the finite interval, we have to send the length of the $x^{6}$ intervals $L \rightarrow 0$. This all has to be done holding $g_{Y M}^{2}=\frac{g_{s}}{m_{s} L}$ fixed. In $11 \mathrm{~d}$ units $g_{Y M}^{2}=\frac{R_{11}}{L}$. In order to keep the interacting gauge theory (fixed $g_{Y M}^{2}$ ) while decoupling the $\mathrm{KK}$ modes $(L \rightarrow 0) R_{11}$ has to go to zero! This is the limit in which the brane setup reduces to $4 \mathrm{~d}$ SYM. But this is the opposite limit of the one we were able to solve, where $R_{11}$ and hence $L$ have to be very large and we can use 11d SUGRA. So we should only expect quantities protected by holomorphy, like the $N=2$ prepotential, which can not depend on the real parameter $R_{11}$, to come out correctly. Indeed it was shown in [76] that unprotected quantities like the 4-derivative terms in the effective action disagree with field theory results.

Non-chiral $N=1$ gauge theories can be obtained by rotating one or several of the NS5-branes such that they intersect by a certain angle. The corresponding continuous parameter can be regarded in field theory as a mass parameter which explicitly breaks $N=2$ supersymmetry down to $N=1$. The M-theory embedding of the non-chiral $N=1$ models, constructed in this way, leads again to supersymmetric 2-cycles, now embedded in the six-dimensional space $\mathbb{R}^{5} \times S^{1}[77,78,79]$. Analysing these curves, the form of the corresponding $N=1$ superpotentials can be derived.

A generic way to construct chiral $N=1$ gauge theories in four dimensions is provided by the brane box models of Hanany and Zaffaroni $[5]^{*_{1}}$, which already appeared in chapter 2. Here one deals with a type IIB configuration of intersecting NS5 and D5branes. We will show that upon a T-duality transformation to the IIA superstring, the M-theory lifting of the chiral $N=1$ brane box configurations leads to supersymmetric 3 -cycles suitably embedded into $\mathbb{C}^{3}$. These supersymmetric 3 -cycles precisely correspond to the $S U(3)$ special Lagrangian calibrations (SLAG) [66, 67], which reduce the original supersymmetry by a factor $1 / 8$. This is just the right amount of supersymmetry breaking

\footnotetext{
${ }^{* 1}$ An alternative, but more restricted construction of chiral $N=1$ models via orientifolds was introduced in [80, 81].
} 
for a generic chiral $N=1$ gauge theory.

In the spirit of [2] one can study the quantum effects in the corresponding gauge theory by analysing the bending of the branes. In particular the $\beta$-function should show up in this approach, since the gauge coupling is encoded in the distance of NS5-branes, which can be measured by the area of the brane box. In this sense the shape of the bent branes directly gives some information about the running gauge coupling. In order to obtain the exact quantum information (or at least the information protected by holomorphy) one must lift the brane configuration to M-theory and use 11d SUGRA to solve it.

Mind ! The boxes of [5] live in IIB theory, so in order to perform the M-theory lift on has to use the relation that IIB on a circle is M-theory on a torus, so we have to compactify one of the common worldvolume directions. Like in the $5 \mathrm{~d}$ case studied in [82] this means that we are really solving the $4 \mathrm{~d}$ theory on $R^{3} \times S^{1}$. In the limit where the area of the M-theory torus shrinks to zero or grows to infinity we recover the $N=1$ $d=4$ and $N=2 d=3$ gauge theories respectively. We will provide evidence that in this way all the holomorphic informations about these gauge theories are encoded in the geometry of a SUSY 3-cycle.

In the next sections we will show how the classical brane boxes relate to supersymmetric 3 -cycles. For the special case of finite theories and theories that satisfy the uniform bending requirement of [11] we are able to perform the lift explicitly. However the corresponding cycles turn out to be superpositions of special 3-cycles that are of the form 2-cycle times line. 


\section{1 $\quad N=1$ Brane Boxes}

Now let us discuss the brane boxes of [5] which can be used to construct $N=1$ supersymmetric gauge theories with chiral matter content. The starting point is now a type IIB superstring with the following branes included (see Fig. 4.2):

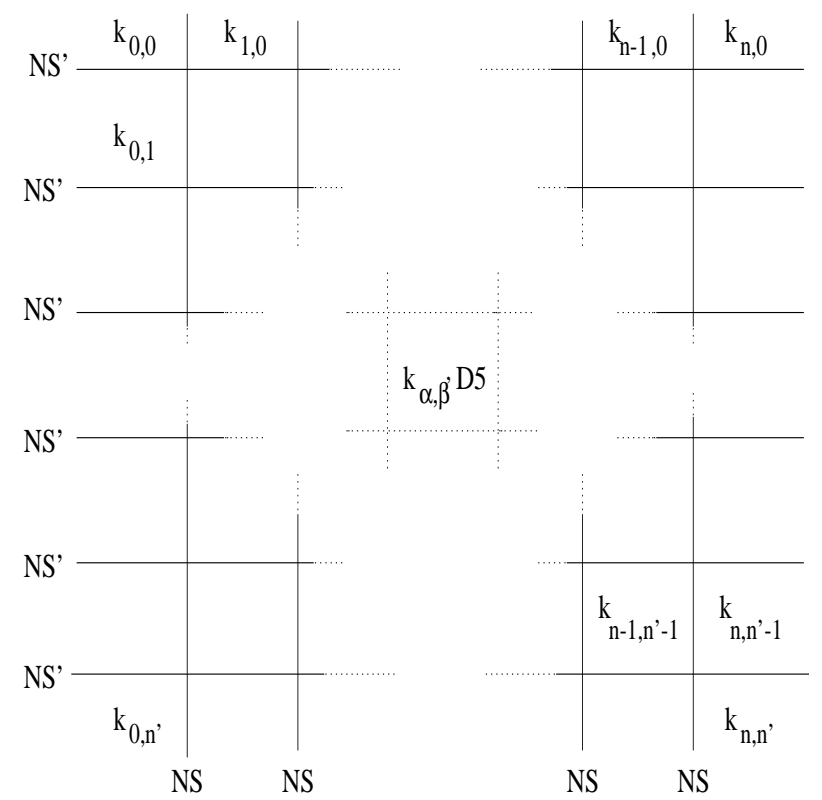

Fig. 4.2: The $\mathrm{N}=1$ brane configuration

- $n$ parallel NS5-branes with world volumes along the (012345)-directions. These branes are fixed at $x^{7}=x^{8}=x^{9}=0$ and are placed at arbitrary positions in $x^{6}$.

- $n^{\prime}$ parallel NS5'-branes with world volumes along the (012367)-directions. The NS5'-branes are fixed at $x^{5}=x^{8}=x^{9}=0$ and are placed at arbitrary positions in $x^{4}$.

- D5-branes with world volumes along the (012346)-directions. The D5-branes can take different position on the NS5-branes in the $x^{5}$-direction and also different positions on the NS5'-branes in the $x^{7}$-direction. Depending on the specific model one likes to discuss, the directions $x^{4}$ and $x^{6}$ can be either uncompactified or periodic (elliptic models). We will concentrate on the non-elliptic models. It follows that the D5-branes are finite in the directions $x^{6}, x^{4}$ in case they are placed inside the 'inner' boxes. However they are semi-infinite in case they end only on one NS5 (NS5') brane ('outer' boxes). 
This brane configuration preserves $1 / 8$ of the original supersymmetry. We see that a generic configurations consists of a grid of $(n+1)\left(n^{\prime}+1\right)$ boxes built by $n$ NS5-branes and $n^{\prime}$ NS5'-branes in the $x^{4}-x^{6}$ plane. We are labelling the boxes by the two indices $\alpha, \alpha^{\prime}$ where $\alpha=0, \ldots, n$ and $\alpha^{\prime}=0, \ldots, n^{\prime}$. The $(n-1)\left(n^{\prime}-1\right)$ 'inner' boxes with $\alpha=1, \ldots, n-1$, $\alpha^{\prime}=1, \ldots, n^{\prime}-1$ have always finite area whereas the remaining 'outer' boxes have infinite size in case of uncompactified directions $x^{4}$ and $x^{6}$.

Now, let $k_{\alpha, \alpha^{\prime}}$ denote the number of D5-branes which are placed in the box $\left[\alpha, \alpha^{\prime}\right]$. The gauge theory of the suspended D5-branes is a generalisation of the orbifold theory described in section 1.3.1 and further investigated in chapter 2. It can be derived using the abstract approach of [14] leading again to a gauge group in four dimensions:

$$
G=\prod_{\alpha=1}^{n-1} \prod_{\alpha^{\prime}=1}^{n^{\prime}-1} S U\left(k_{\alpha, \alpha^{\prime}}\right) .
$$

The associated classical gauge coupling constants are given by the area of the corresponding box $\left[\alpha, \alpha^{\prime}\right]$ :

$$
\frac{1}{g_{\alpha, \alpha^{\prime}}^{2}}=\frac{\left(x_{\alpha+1}^{4}-x_{\alpha}^{4}\right)\left(x_{\alpha^{\prime}+1}^{6}-x_{\alpha^{\prime}}^{6}\right)}{g_{s}} .
$$

The matter content of the model consists of three types of chiral $N=1$ representations, well known from section 1.3.1. The 'horizontal' chiral bifundamentals $H_{\alpha, \alpha^{\prime}}$ in the representations $\left(k_{\alpha, \alpha^{\prime}}, \bar{k}_{\alpha+1, \alpha^{\prime}}\right)$ of $S U\left(k_{\alpha, \alpha^{\prime}}\right) \times S U\left(k_{\alpha+1, \alpha^{\prime}}\right)$, the 'vertical' chiral bifundamentals $V_{\alpha, \alpha^{\prime}}$ in the representations $\left(k_{\alpha, \alpha^{\prime}}, \bar{k}_{\alpha, \alpha^{\prime}+1}\right)$ of $S U\left(k_{\alpha, \alpha^{\prime}}\right) \times S U\left(k_{\alpha, \alpha^{\prime}+1}\right)$ and finally the 'diagonal' chiral fields $D_{\alpha, \alpha^{\prime}}$ in the representations $\left(k_{\alpha, \alpha^{\prime}}, \bar{k}_{\alpha-1, \alpha^{\prime}-1}\right)$ of $S U\left(k_{\alpha, \alpha^{\prime}}\right) \times S U\left(k_{\alpha-1, \alpha^{\prime}-1}\right)$ $\left(\alpha, \alpha^{\prime}>1\right)$. In this context the groups $S U\left(k_{\alpha, \alpha^{\prime}}\right)$ with $\alpha=0, n$ or $\alpha^{\prime}=0, n^{\prime}$ act as global flavour symmetries if the directions $x^{4}$ and $x^{6}$ are uncompactified. Note that the choices for the $k_{\alpha, \alpha^{\prime}}$ are severely constrained by the requirement of absence of anomalies. If all three types of matter multiplets are present then there exists a classical superpotential of the following form:

$$
W=\sum_{\alpha, \alpha^{\prime}} H_{\alpha, \alpha^{\prime}} V_{\alpha+1, \alpha^{\prime}} D_{\alpha+1, \alpha^{\prime}+1}-\sum_{\alpha, \alpha^{\prime}} V_{\alpha, \alpha^{\prime}} H_{\alpha, \alpha^{\prime}+1} D_{\alpha+1, \alpha^{\prime}+1}
$$

So far we have only discussed the classical field theory. Of course it is essential to understand the quantum features as well. Chiral $N=1$ exhibit a huge variety of interesting quantum phenomena. Especially the generic theory will have an anomaly which should show up as an inconsistency of the brane box as a string background. In these general cases the bending of the brane boxes isn't well understood yet, but some special cases can be analysed.

It is clear that the bending of the NS5 and NS5'-branes depends on the number $k_{\alpha, \alpha^{\prime}}$ of D5-branes in each box. A very special class of $N=1$ gauge theories is given by the 
finite models (further discussed in chapter 5 ) for which all $\beta$-functions and all anomalous dimensions vanish to all orders in perturbation theory [83, 84]. This condition includes the vanishing of the one-loop $\beta$-functions. In the brane picture complete finiteness means that all NS5 and NS5'-branes do not bend at all, i.e. if the number of D5-branes in every box is the same [84]. Then obviously, $N_{f}=3 N_{c}$ for every gauge group factor, and the one-loop $\beta$-functions are zero.

The corresponding brane setup consists of several branes put on top of each other. Each of the branes preserves $1 / 2$ of the supersymmetries, together they still preserve $1 / 8$, so the intersection is BPS. This ensures that the static branes don't exert any force on each other. We can freely move the constituent branes since they don't feel the presence of the other branes at all. Motions of the branes in the 46 plane corresponds to changing the areas of the various boxes and hence to changing the gauge couplings. Taking NS5 (NS5') branes away along the $x^{7}\left(x^{5}\right)$ direction destroys the box structure and corresponds to turning on Fayet-Iliopoulos terms.

Another special situation is that of uniform bending. The condition of uniform bending was first introduced by [11]. There it was argued to be necessary for consistency. As we will see this is too stringent. However uniform bent setups are very special and allow for a precise treatment of the quantum properties. To motivate the uniform bending requirement consider the basic cross configuration of Fig. 4.3.

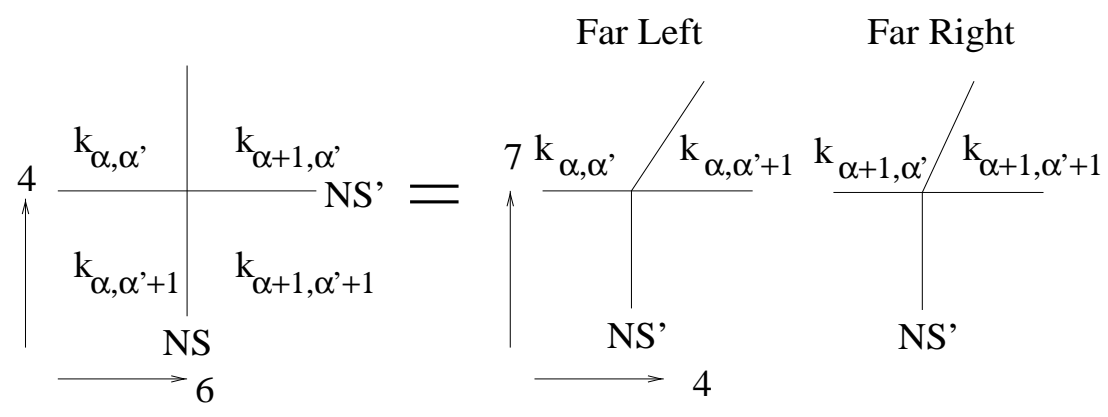

Fig. 4.3: Basic cross configuration

For $x^{6} \rightarrow-\infty$ (to the far left) the effects of the NS5-brane on the bending of the NS5'brane should be negligible. The D5 ending on the NS5'-brane just looks like a 5 d gauge theory with 8 supercharges and leads to the standard linear bending [85]. The slope of the bending is given by the difference of branes ending from either side, hence

$$
\text { slope }_{x^{6} \rightarrow-\infty}=k_{\alpha, \alpha^{\prime}}-k_{\alpha, \alpha^{\prime}+1} \text {. }
$$

For the same reason we will have linear bending to the far right, that is for $x^{6} \rightarrow \infty$, the slope this time given by

$$
\text { slope }_{x^{6} \rightarrow \infty}=k_{\alpha+1, \alpha^{\prime}}-k_{\alpha+1, \alpha^{\prime}+1} .
$$


The observation of [11] was that if

$$
k_{\alpha, \alpha^{\prime}}-k_{\alpha, \alpha^{\prime}+1}=k_{\alpha+1, \alpha^{\prime}}-k_{\alpha+1, \alpha^{\prime}+1}
$$

the bending on the far left is the same as on the far right and one may expect that the shape of the NS5' in fact does not change at all as a function of $x^{6}$. In [10] it was observed that the most general setup compatible with condition (4.8) can be achieved by "sewing" together $N=2$ models, that is we take branes corresponding to $5 \mathrm{~d}$ gauge theory built out of NS5 and D5-branes and move them on top of a similar setup build out of NS5' and D5-branes*2, as illustrated in Fig. 4.4.
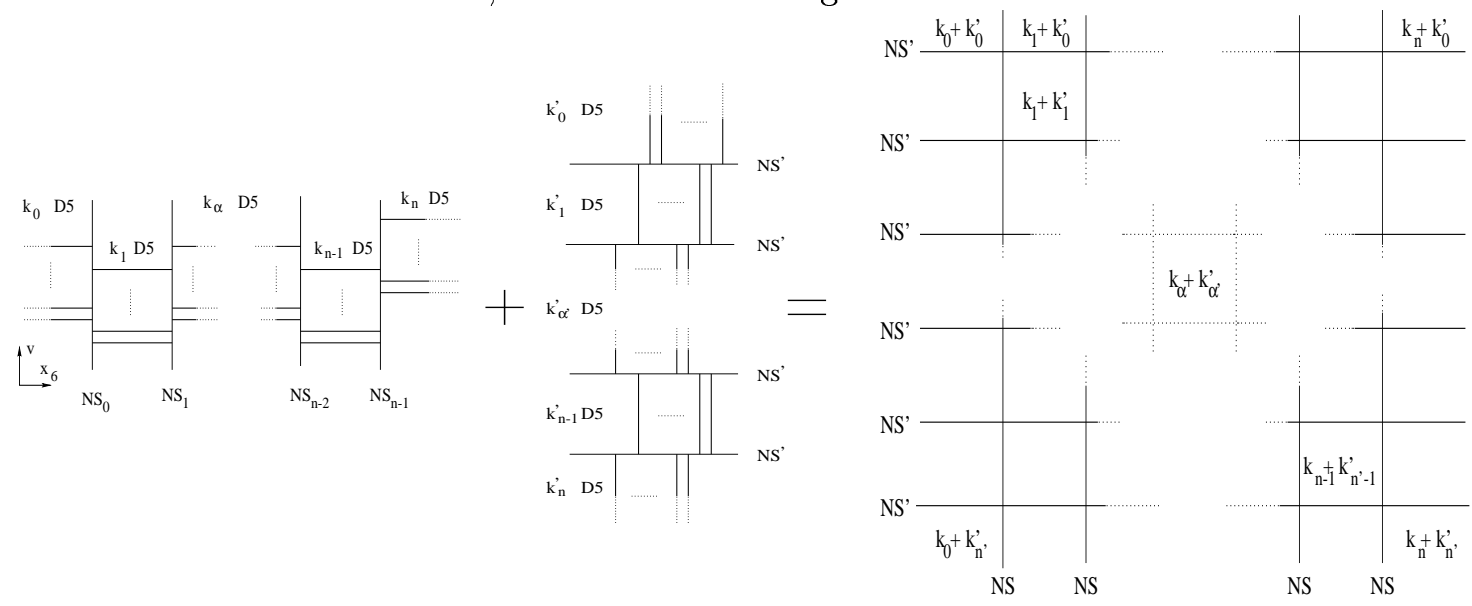

Fig. 4.4: Sewing of $\mathrm{N}=2$ models.

This sewing can be taken quite literally: as in the finite case there is a no force condition between the constituent pieces, since their intersection still preserves some supersymmetry. So we are free to move them independently. These deformations should correspond to marginal couplings in the field theory. Since we are not just tuning the distance between two NS5-branes but are actually moving around compound systems, these marginal operators won't just be the gauge coupling as in the finite case but will also involve the superpotential couplings. Using the methods of [83], the authors of [84] were indeed able to show that the field theory has these marginal operators if the conditions of eq. (4.8) are satisfied for all boxes. Since the subsystems don't influence each other, the exact bending is given in terms of the linear bending of the subsystems. All uniform bent systems are anomaly free.

\footnotetext{
${ }^{*_{2}}$ In [10] they also allowed sewing in a third kind of $N=2$ system connected to diagonal lines in the box setup. This doesn't lead to uniform bent models anymore and should hence be treated separately.
} 


\subsection{T-Duality, M-Theory Embedding and the Emergence of the 3-Cycles}

Now let us describe the the strong coupling limit of the $N=1$ via embedding the brane boxes into M-theory. Since our original brane configurations is in the type IIB string, we have first to perform a T-duality transformation to the type IIA superstring before we can perform the M-theory embedding. We do not want to touch the NS5 and NS5'branes, and we also do not want to create any D6-branes; therefore we will T-dualise over one of the spatial directions common to all branes. To be specific we now assume that $x^{3}$ is periodic with radius $R_{3}^{I I B}$ and we perform the T-duality with respect to the $x^{3}$-direction. This leads to the following brane configuration:

- $n$ parallel NS5-branes with world volumes along the (012345)-directions. These branes are fixed at $x^{7}=x^{8}=x^{9}=0$ and are placed at arbitrary positions in $x^{6}$.

- $n^{\prime}$ parallel NS5'-branes with world volumes along the (012367)-directions. The NS5'-branes are fixed at $x^{5}=x^{8}=x^{9}=0$ and are placed at arbitrary positions in $x^{4}$.

- D4-branes with world volumes along the (01246)-directions. These D4-branes take different $x^{5}$ positions on the NS5-branes and also different $x^{7}$ positions on the NS5'branes. In addition the D4-branes can have arbitrary positions in the compactified spatial $x^{3}$-direction.

This configuration preserves like before $1 / 8$ of the original supersymmetries and corresponds to a three-dimensional gauge theory with $N=2$ space-time supersymmetry. The three-dimensional gauge theory can be simply obtained from the four-dimensional $N=1$ models by circle compactification on $S^{1}$ in the $x^{3}$-direction. In the decompactification limit, $R_{3}^{I I B} \rightarrow \infty$, the four-dimensional $N=1$ gauge theories are rediscovered. On the hand, for $R_{3}^{I I B} \rightarrow 0$, the theory is truly three-dimensional. Note that in three dimensions, a new Coulomb branch can be opened, since the three-dimensional vector multiplets contain one real scalar degree of freedom. The corresponding modulus $v$ is associated in the brane picture with the positions of the D4-branes in the $x^{3}$-direction. The three-dimensional gauge coupling is classically related to the $4 \mathrm{~d}$ gauge coupling as $1 / g_{3}^{2}=R_{3}^{I I B} / g_{4}^{2}$. So in the limit $R_{3}^{I I B} \rightarrow \infty$ one must send $g_{3} \rightarrow 0$ in order to have a finite coupling $g_{4}$. The scalar field in the vector multiplet live on a dual circle with radius $R_{3}^{I I A}=1 / R_{3}^{I I B}$. So in the $4 \mathrm{~d}$ limit, $R_{3}^{I I A} \rightarrow 0$, one has to integrate out the fields with masses of order $v$ corresponding to the Coulomb branch. In this way we can regard $v$ as the parameter which sets the scale $\Lambda$ of the four-dimensional gauge theory. So in order to determine the logarithmic 'running' of the four-dimensional gauge coupling $g_{4}$ in terms of $\Lambda, 1 / g_{4}^{2}=b^{N=1} \log \Lambda$, we will be in particular interested in the bending of the coordinates 
$x^{4}$ and $x^{6}$ in terms of $x^{3}$. (This precisely corresponds to the logarithmic running of $x^{6}$ in terms of the Higgs field vev $v=x^{4}+i x^{5}$ in case of the $N=2$ brane models.) This will be further discussed in section 4.5.2. Note that in the 3-dimensional limit, $R_{3}^{I I B} \rightarrow 0$, the pure Yang-Mills gauge theory has no stable supersymmetric groundstate unlike the $4 \mathrm{~d}$ theory. Many more details of the dynamics and superpotentials of three-dimensional, $N=2$ supersymmetric gauge theories can be found in [86].

After the duality in $x^{3}$ we are now ready for lifting the above configuration to M-theory by adding the period direction $x^{10}$ with radius $R_{11}$.

Like in the $N=2$ case the singular intersections of the NS5, NS5' and D4-branes are described in M-theory by a single smooth M5-brane. Asymptotically, this M5-brane takes the shape of the classical IIA branes:

- The NS5-branes asymptotically correspond to M5-branes which extend in the (012345)-space and take different positions in the $x^{6}, x^{7}, x^{8}, x^{9}$ and $x^{10}$ directions.

- The NS5'-branes asymptotically look like M5-branes with world volumes along the (012367)-space and positions in $x^{4}, x^{5}, x^{8}, x^{9}$ and $x^{10}$.

- Finally, the asymptotics of the D4-branes is given by M5-branes with world volumes in $x^{0}, x^{1}, x^{2}, x^{4}, x^{6}$ and $x^{10}$ and positions in $x^{3}, x^{5}, x^{7}, x^{8}$ and $x^{9}$.

So all branes have common world volumes in the (012)-space and are all located at $x^{8}=x^{9}=0$. Therefore, to characterise the M-theory configurations we have to focus on the six-dimensional space spanned by the coordinates $x^{3}, x^{4}, x^{5}, x^{6}, x^{7}$ and $x^{10}$. Each asymptotic brane fills three particular directions in this space. This means that the general embedding of the M5-branes is described by a three-dimensional submanifold in $\mathbb{R}^{4} \times T^{2}$. Supersymmetry requires this to be a supersymmetric 3-cycle. In the language of [66] this is called a $S U(3)$ special Lagrangian calibration (SLAG) which breaks $1 / 8$ of the supersymmetries.

Let us again briefly consider the validity of our approach. In $11 \mathrm{~d}$ units the $3 \mathrm{~d}$ gauge coupling is given by $g_{Y M}^{2}=\frac{R_{11}}{A}$ where $A$ is the area of the box. As in the $N=2$ case there are two distinct limits if we want to keep $g_{Y M}^{2}$ fixed: for small $R_{11}$ and $A$ the KK modes decouple and the brane setup reproduces the gauge theory. For large $R_{11}$ we can solve using 11d SUGRA, that is by solving for the SUSY 3-cycle. However, as for $N=2$, all holomorphic quantities should be encoded in the geometry of the 3-cycle. That is of the terms in the 2-derivative approximation of the effective action, the holomorphic gauge coupling and the superpotential should be encoded in the 3-cycle, whereas the Kähler potential probably escapes our control. 


\subsection{Brane Cubes and M-Theory}

As already mentioned in the original work of [5] the idea of brane boxes can naturally be extended to brane cubes and brane hypercubes. Each time we add one more NS5brane with yet another orientation breaking another half of the supersymmetry. The D-brane ends on these new NS5-branes as well, so that the brane spans a $3 \mathrm{~d}$ cube or $4 \mathrm{~d}$ hypercube instead of the $2 \mathrm{~d}$ box we considered so far. Let us briefly discuss, how these configurations are lifted to M-theory. We will find that the situation is especially interesting in the case of brane cubes, where one can find two distinct models, one with chiral and one with non-chiral SUSY.

For simplicity let us consider the brane cubes directly on the IIA side with the D4-brane suspended between the NS5-branes. This is the setup that lifts to M-theory in a straight forward fashion. The third NS5-brane that we add should have a fixed $x^{2}$ position, so that the D4-brane is finite in this direction as well as in $x^{4}$ and $x^{6}$. There are two distinct possibilities to do so. The first is to add an NS5"-brane along 014567. This is the setup considered in [87]. It leads to a chiral $N=(2,0)$ supersymmetric gauge theory in $d=2$. Rotations in 89 space give rise to the $U(1)_{R}$ symmetry. The dual orbifold consists of D1-branes living on top of an $\mathbb{C}^{4} / \Gamma$ orbifold, where $\Gamma$ is a subgroup of $S U(4)$. By the same reasoning as for the brane box we find that this chiral brane cube should lift to M-theory via a SUSY 4-cycle in the 7d 234567 space, that is via a SUSY 4-cycle associated with $G_{2}$ holonomy.

The second possibility is to have the NS5"-brane in 012468 . This leaves us with $N=(1,1)$ in 2 dimensions. Since this time all three types of NS5-branes have a common direction $\left(x^{3}\right)$ we can perform a T-duality to type IIB as for the box, leading to a $3 \mathrm{~d} N=1$ theory. This time the dual orbifold is a $G_{2}$ orbifold while the lift to M-theory has to be performed via an $S U$ (4) SLAG 4-cycle in $\mathbb{C}^{4}$. Therefor this non-chiral cube requires the same techniques as the SLAG 3-cycle. By simply adding another NS5 along 23469 we find the brane hypercube and its lift via an $S U(5)$ SLAG.

\subsection{Supersymmetric 3-Cycles}

Recall the characteristic $N=1$ brane configuration:

\begin{tabular}{|l|lllllllll|}
\hline NS5 : & 0 & 1 & 2 & 3 & 4 & 5 & & & \\
NS5: & 0 & 1 & 2 & 3 & & & 6 & 7 & \\
D4 : & 0 & 1 & 2 & & 4 & & 6 & & \\
\hline
\end{tabular}

Tab. 4.3: brane configuration.

As explained in appendix $\mathrm{C}$ the precise definition of a supersymmetric 3 -cycle requires the 
introduction of a complex structure into $\mathbb{R}^{6}$. This leads to a splitting of the coordinates into two sets $q_{i}+i \cdot p_{i}$, which is a reflection of the fact that $\mathbb{R}^{6}$ can be regarded as the phase space of a Hamiltonian system. Therefore we divide the variables into space $\left(q_{i}\right)$ and momentum $\left(p_{i}\right)$ coordinates respectively. Since the NS5-branes together with the D4-branes build a $N=2$ subsystem, two conjugated $(q, p)$ pairs are given by $\left(q_{1}, p_{1}\right)=$ $\left(x^{10}, x^{3}\right)$ and $\left(q_{2}, p_{2}\right)=\left(x^{6}, x^{5}\right)$. Then a single NS5-brane as well as a single D4-brane is automatically a supersymmetric 3-cycle, namely a supersymmetric 2-cycle in the $x^{3}$ $x^{5}-x^{6}-x^{10}$ space times the line $x^{7}=$ const in $x^{4}-x^{7}$ space. The last pair of coordinates is fixed by the requirement that also the NS5'-brane is a supersymmetric 3-cycle: $\left(q_{3}, p_{3}\right)=$ $\left(x^{4}, x^{7}\right)$. In summary, the complex structure of $\mathbb{C}^{3}$ takes the following form:

$$
\begin{aligned}
& u^{1}=x^{10}+i \cdot x^{3} \\
& u^{2}=x^{6}+i \cdot x^{5} \\
& u^{3}=x^{4}+i \cdot x^{7}
\end{aligned}
$$

Now we can work out the supersymmetric 3-cycle conditions (see appendix C.1) on the three functions

$$
\begin{aligned}
& f^{1}=f\left(x^{3}, x^{4}, x^{5}, x^{6}, x^{7}, x^{10}\right), \\
& f^{2}=g\left(x^{3}, x^{4}, x^{5}, x^{6}, x^{7}, x^{10}\right), \\
& f^{3}=h\left(x^{3}, x^{4}, x^{5}, x^{6}, x^{7}, x^{10}\right) .
\end{aligned}
$$

First, the three Poisson brackets are given by the following set of equations:

$$
\begin{aligned}
& 0 \equiv\{f, g\}=f_{10} g_{3}-f_{3} g_{10}+f_{4} g_{7}-f_{7} g_{4}+f_{6} g_{5}-f_{5} g_{6}, \\
& 0 \equiv\{f, h\}=f_{10} h_{3}-f_{3} h_{10}+f_{4} h_{7}-f_{7} h_{4}+f_{6} h_{5}-f_{5} h_{6}, \\
& 0 \equiv\{g, h\}=g_{10} h_{3}-g_{3} h_{10}+g_{4} h_{7}-g_{7} h_{4}+g_{6} h_{5}-g_{5} h_{6} .
\end{aligned}
$$

The equation $\operatorname{det} N \equiv 1$ takes the following form:

$$
\begin{aligned}
0 & \equiv\left(f_{4} g_{6}-f_{7} g_{5}-f_{6} g_{4}+f_{5} g_{7}\right) h_{10}+\left(g_{10} f_{6}-g_{3} f_{5}-f_{10} g_{6}+f_{3} g_{5}\right) h_{4} \\
& +\left(f_{10} g_{4}-f_{3} g_{7}-g_{10} f_{4}+g_{3} f_{7}\right) h_{6}+\left(g_{3} f_{4}+g_{10} f_{7}-f_{10} g_{7}-f_{3} g_{4}\right) h_{5} \\
& +\left(f_{10} g_{5}+f_{3} g_{6}-g_{3} f_{6}-g_{10} f_{5}\right) h_{7}+\left(f_{6} g_{7}-f_{7} g_{6}-f_{4} g_{5}+f_{5} g_{4}\right) h_{3} .
\end{aligned}
$$

For a supersymmetric 3 -cycles these four equations must be zero modulo the ideal of vanishing functions determined by $f, g$ and $h$.

One particular class of solutions for these equations is of course given by all 3-cycles which are a supersymmetric 2-cycle in the $x^{3}-x^{5}-x^{6}-x^{10}$ space times the line $x^{7}=$ const in $x^{4}-x^{7}$ space: $\Sigma^{(3)}=\Sigma^{(2)} \times \mathbb{R}$. The corresponding choice of functions is

$$
\begin{aligned}
f & =\Re \mathfrak{e} F\left(x^{3}+i x^{5}, x^{6}+i x^{10}\right), \\
g & =\Im \mathfrak{m} F\left(x^{3}+i x^{5}, x^{6}+i x^{10}\right), \\
h & =x^{7} \text { - const. }
\end{aligned}
$$


As a first and very simple check we can verify that flat, parallel M5-branes in their three possible asymptotic limits, namely being NS5, NS5' or D4-branes, are indeed supersymmetric 3-cycles. For example consider the $n$ parallel NS5-brane, positioned at $x_{i}^{6}, x_{i}^{7}$ and $x_{i}^{10}(i=1, \ldots, n)$. Hence the three functions $f, g$ and $h$ are given as

$$
\begin{aligned}
f & =\prod_{i=1}^{n}\left(x^{6}-x_{i}^{6}\right), \\
g & =\prod_{i=1}^{n}\left(x^{7}-x_{i}^{7}\right), \\
h & =\prod_{i=1}^{n}\left(x^{10}-x_{i}^{10}\right) .
\end{aligned}
$$

It is easy to show that all eqs. (4.9) and (4.10) are identically zero. The same is of course true for $n^{\prime}$ parallel NS5'-branes and $k$ parallel D4-branes. In the following sections we will discuss more complicate brane intersections and bent brane configurations.

\subsection{Supersymmetric 3-Cycles for $N=1$ Brane Boxes}

\subsubsection{Branes as Quaternionic Coordinates}

In the following sections we like to construct the defining equations $f, g$ and $h$ for those supersymmetric 3-cycles, which correspond to intersecting NS5, NS5' and D4-branes, and in particular for those, which correspond to $N=1$ brane box configurations with uniform bending. For this purpose we would like to introduce three types of 'coordinates', called $s, s^{\prime}$ and $v$, which denote the asymptotic positions in $\mathbb{C}^{3}$ of the NS5, NS5' and D4branes respectively. These 'coordinates' should be one the same footing as the complex variables $s=x^{6}+i x^{10}$ and $v=x^{4}+i x^{5}$ of the $N=2$ (NS5-D4) brane configurations.

To achieve this aim we will now extend the dimension of the space by including also the directions $x^{2}$ and $x^{8}$. This means that we are now dealing with supersymmetric 4 -cycles which are embedded into the space $\mathbb{C}^{4}$, which is spanned by the directions $(2,3,4,5,6,7,8,10)$. All our branes now fill 4 dimensions of this eight dimensional space: their world volumes completely fill $x^{2}$, and they are all positioned at $x^{8}=0$. That means that the 4-cycles, which correspond to the brane boxes of the NS5, NS5' and D4-branes are in fact nothing else than supersymmetric 3-cycles times the line $x^{8}=0$. As discussed in detail above, we could add yet another type of NS5-branes, called NS5"branes, with world volumes along the $(3,4,6,8)$-directions and positions in the $(2,5,7,10)$ space. Considering intersections of all four types of branes (NS5-NS5'-NS5"-D4) one can construct brane cube models, where the D4-branes are now finite in the directions $x^{2}, x^{4}$ and $x^{6}$. These brane cubes provide two-dimensional gauge theories with $(1,1)$ 
supersymmetry. A generic brane cube configuration corresponds to a supersymmetric 4 -cycle, which is not a direct product of supersymmetric 3-cycle times $\mathbb{R}$.

The positions of the branes in $\mathbb{C}^{4}$ can now nicely described by introducing quaternionic numbers. A general quaternion $q \in \mathbb{H}$ has the structure

$$
q=q^{0} \sigma_{0}+q^{1} \sigma_{1}+q^{2} \sigma_{2}+q^{3} \sigma^{3},
$$

where $\sigma_{0}=\mathbb{1}_{2}$ and the $\sigma_{i}(i=1,2,3)$ are the Pauli matrices, satisfying $\sigma_{i} \sigma_{j}=\epsilon_{i j k} \sigma_{k}$. Clearly, a quaternion is zero, $q=0$, if all its components $q_{i}(i=0, \ldots, 3)$ are vanishing. Alternatively, we can also define the quaternions via two complex numbers $z_{1}=q^{0}+i q^{1}$ and $z_{2}=q^{2}-i q^{3}$ as $q=z_{1}+j z_{2}$, where $i=\sigma_{1}, j=\sigma_{2}$ and $k=i \cdot j=\sigma_{3}$.

Now we can associate to every brane a particular quaternion $q$, which describes its asymptotic position in $\mathbb{C}^{4}$, and hence is a function of the position variables of every brane:

$$
\begin{aligned}
& \text { NS5: } \quad q_{N S 5}=q\left(x^{6}, x^{7}, x^{8}, x^{10}\right), \\
& N S 5^{\prime}: \quad q_{N S 5^{\prime}}=q\left(x^{4}, x^{5}, x^{8}, x^{10}\right) \text {, } \\
& D 4: \quad q_{D 4}=q\left(x^{3}, x^{5}, x^{7}, x^{8}\right) .
\end{aligned}
$$

The four defining equations $f^{m}\left(x^{2}, x^{3}, x^{4}, x^{5}, x^{6}, x^{7}, x^{8}, x^{10}\right)(m=1, \ldots, 4)$ for the 4-cycle can be now simply written in terms of a single quaternionic function function $F\left(q_{N S 5}, q_{N S 5^{\prime}}, q_{D 4}\right)$ :

$$
F\left(q_{N S 5}, q_{N S 5^{\prime}}, q_{D 4}\right)=f^{1}\left(x^{i}\right)+i f^{2}\left(x^{i}\right)+j f^{3}\left(x^{i}\right)+k f^{4}\left(x^{i}\right) .
$$

Of course, for a general function $F\left(q_{N S 5}, q_{N S 5^{\prime}}, q_{D 4}\right)$ one still has to verify whether the 4-cycle is supersymmetric. This is not automatic unlike the case of the supersymmetric 2-cycles, where every holomorphic function corresponds to a supersymmetric 2-cycle. Specifically, as discussed in section C.1, the supersymmetry conditions are given by the requirement that six Poisson brackets $\left\{f^{m}, f^{n}\right\}$ plus (det $N-1$ ) have to vanish (modulo the ideal of vanishing functions determined by the zero locus of the $f^{m}$ ). In addition, since we want the supersymmetric 4-cycle $\Sigma^{(4)}$ to be of the form $\Sigma^{(4)}=\Sigma^{(3)} \times \mathbb{R}_{x^{8}=0}$, the function $F\left(s, s^{\prime}, v\right)$ has to be chosen in such a way that the common zero locus of the $f^{m}$ always contains the line $x^{8}=0$. In principle it is also possible to obtain the three 3 -cycle equations $f, g$ and $h$ by solving one of the four equations $f^{m}$ with respect to $x^{8}$ and substituting the result into the remaining equations.

To understand this procedure of constructing supersymmetric 3-cycles let us first consider case of classical brane configurations which are not bent by quantum effects. To describe flat branes we introduce the following quaternionic coordinates in analogy to 
the complex variables $s$ and $v^{*_{3}}$ :

$$
\begin{aligned}
N S 5: & s=x^{6}+i x^{10}+j x^{7}-k x^{8} \\
N S 5^{\prime}: & s^{\prime}=x^{4}+i x^{5}+j x^{10}-k x^{8} \\
D 4: & v=x^{3}+i x^{5}+j x^{7}-k x^{8} .
\end{aligned}
$$

A single NS5-brane is a supersymmetric 4-cycle simply defined by the equation $F=s=0$ and likewise for the other branes. Next consider the triple intersection of $n$ parallel NS5-branes with $n^{\prime}$ parallel NS5'-branes and $k$ parallel D4-branes. This configuration corresponds to un-bent NS5 and NS5'-branes. In the language of field theory it leads to finite $N=1$ gauge theories. The associated quaternionic function $F$ is given by the following polynomial:

$$
F\left(s, s^{\prime}, v\right)=\prod_{i=1}^{n}\left(s-s_{i}\right) \prod_{j=1}^{n^{\prime}}\left(s^{\prime}-s_{j}^{\prime}\right) \prod_{l=1}^{k}\left(v-v_{l}\right) .
$$

Here $s_{i}, s_{j}^{\prime}$ and $v_{l}$ are constant quaternionic numbers with zero $\sigma_{3}$-component, which denote the positions of the three types of branes. It is straightforward to show that this function $F$ corresponds to a supersymmetric 4-cycle.

\subsubsection{Uniform Bending - Sewing of $N=2$ Models}

Now we will construct the supersymmetric 3-cycles which correspond to those $N=$ 1 brane boxes which can be obtained via the sewing or superposition of two $N=2$ subsystems. As explained in section 4.1, this means that all the NS5-branes as well as all the NS5'-branes are bent in an uniform way.

In general, the bending of the NS5 and NS5'-branes should be parametrised by the $x^{3}$ position of the D4-branes, where $x^{3}$ is nothing else that the parameter which is associated to the Coulomb branch in three dimensions. In addition, we roughly expect that the bending of the NS5-brane is encoded in the functions $x^{6}\left(x^{3}\right)$ and $x^{10}\left(x^{3}\right)$, and analogously, the bending of the NS5'-branes is determined by $x^{4}\left(x^{3}\right)$ and $x^{10}\left(x^{3}\right)$. Since $x^{3}$ takes in four dimensions the role of $\Lambda_{Q C D}, x^{4}, x^{6}$ and $\cos x^{10}$ ( $x^{10}$ is periodic!) should be logarithmic functions of $x^{3}$.

For the case of uniform bending we can be much more explicit. Consider first the uniform bending of the NS5-brane caused by $k$ D4-branes. From the $N=2$ models we know that the perturbative bending is described by a two dimensional Laplace equation with the holomorphic, logarithmic solution $x^{6}+i x^{10}=k \log \left(x^{3}+i x^{5}\right)$. In the same way, for the other $N=2$ subsystem, NS5' $-\mathrm{k}^{\prime} \mathrm{D} 4$, the following perturbative solution for the bending holds: $x^{4}+i x^{10}=k^{\prime} \log \left(x^{7}+i x^{3}\right)$. This behaviour now suggest that we define the following

\footnotetext{
${ }^{*_{3}}$ The NS5"-brane corresponds to the quaternion $s^{\prime \prime}=x^{10}+i x^{5}+j x^{7}-k x^{2}$.
} 
quaternionic coordinates which describe the asymptotic positions of the bent branes in a correct way:

$$
\begin{aligned}
N S 5: & t & =e^{x^{6}} \cos x^{10}+i e^{x^{6}} \sin x^{10} \\
N S 5^{\prime}: & t^{\prime} & =e^{x^{4}} \cos x^{10}+j e^{x^{4}} \sin x^{10} \\
D 4: & v & =x^{3}+i x^{5}+j x^{7}-k x^{8} .
\end{aligned}
$$

Sewing together the perturbative bending of the two $N=2$ subsystems provides us with the following quaternionic function for the supersymmetric 3-cycle, which corresponds to the simple brane box shown in Fig. 4.5:

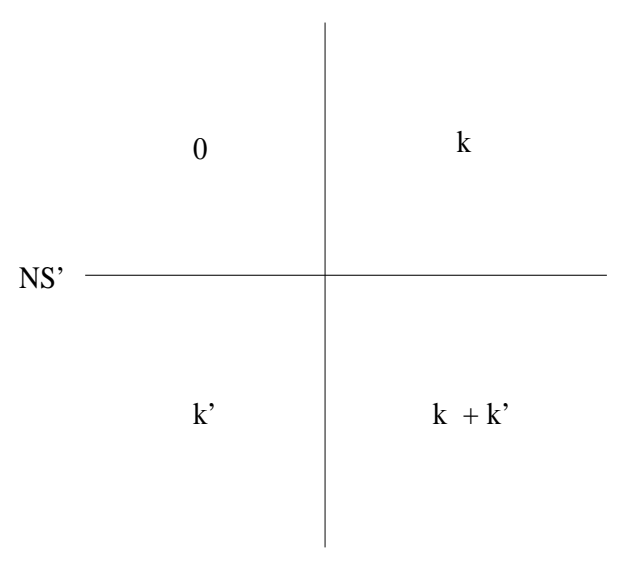

NS

Fig. 4.5: A simple brane box with uniform bending

For $k=k^{\prime}=1$ the quaternionic function simply takes the form

$$
F\left(t, t^{\prime}, v\right)=[t-v] \cdot\left[t^{\prime}-v\right]=0
$$

Similar one can write down an expression for arbitrary $k$ and $k^{\prime}$. It is possible to show that this function satisfies the conditions for a supersymmetric cycle. The vanishing locus which is defined by $F\left(t, t^{\prime}, v\right)$ is a true 3 -cycle; it consists out of two branches, namely the superposition of the curve $t-v^{k}=0$, which is a 2-cycle in the $3-5-6-10$ directions times the $x^{4}$-axis, together with the curve $t^{\prime}-v^{k^{\prime}}=0$, which represents a 2-cycle, now in the directions $3-4-7-10$ times the $x^{6}$-axis.

After having understood the most simple $N=1$ brane box with uniform bending (see Fig. 4.5) we can now construct the non-perturbative, supersymmetric 3-cycle equations which describe the generic $N=1$ brane box with uniform bending situation. It is given by the superposition of two $N=2$ subsystems: the first one consists out of $n$ NS5branes with $k_{\alpha}$ D4-branes suspended between the NS5-branes (see Fig. 4.1). The second 
$N=2$ subsystem has the same structure, but now $n^{\prime}$ NS5'-branes with $k_{\alpha^{\prime}}^{\prime}$ suspended D4-branes. After sewing together these two subsystems, the $N=1$ brane box has the form shown in Fig. 4.4. Now recall that, non-perturbatively, an $N=2$ system of the type in Fig. 4.1 is characterised by a complex 2-cycle polynomial

$$
\Sigma_{n, k_{\alpha}}^{(2)}: \quad F_{n, k_{\alpha}}(t, v)=p_{k_{0}}(v) t^{n}+p_{k_{1}}(v) t^{n-1}+\ldots+p_{k_{n-1}}(v) t+p_{k_{n}}(v) .
$$

Then the sewing procedure simply corresponds to the multiplication of the two $N=2$ polynomials, where we replace the complex variables by the corresponding quaternionic variables. In this way we get a supersymmetric 3-cycle which consists out of two branches, namely the direct sum

$$
\Sigma_{n, n^{\prime}, k_{\alpha}, k_{\alpha^{\prime}}^{\prime}}^{(3)}=\left(\Sigma_{n, k_{\alpha}}^{(2)} \times \mathbb{R}\right) \oplus\left(\Sigma_{n^{\prime}, k_{\alpha^{\prime}}^{\prime}}^{(2)} \times \mathbb{R}\right) .
$$

Note that the two superposed 3-cycles have a common volume in the 3-10 space. In general the quaternionic 3-cycle equations will have the following structure:

$$
\Sigma^{(3)}: \quad F\left(t, t^{\prime}, v\right)=\left[p_{k_{0}}(v) t^{n}+\ldots+p_{k_{n}}(v)\right] \cdot\left[p_{k_{0}^{\prime}}(v) t^{\prime n}+\ldots+p_{k_{n^{\prime}}^{\prime}}(v)\right] .
$$

This expression can be expanded and one obtains a polynomial of the following structure:

$$
F\left(t, t^{\prime}, v\right)=\sum_{\alpha=0}^{n} \sum_{\alpha^{\prime}=0}^{n^{\prime}} p_{k_{\alpha}}(v) p_{k_{\alpha^{\prime}}^{\prime}}(v) t^{n-\alpha} t^{n^{\prime}-\alpha^{\prime}} .
$$

Note that the degree of the polynomial in $v$ in front of each term $t^{n-\alpha} t^{n^{\prime}-\alpha^{\prime}}$ precisely agrees with the number of D4-branes in each box $\left[\alpha, \alpha^{\prime}\right]$.

For example the sewing of two pure $N=2$ gauge theories with $G=S U(k)$ and $G^{\prime}=S U\left(k^{\prime}\right)$ leads to a $N=1$ gauge theory with $N_{c}=N_{f}=k+k^{\prime}$ (see Fig. 4.6).

The corresponding 3-cycle equations are then simply given in terms of the product of two Seiberg-Witten elliptic curves of genus $(k-1)$ resp. $\left(k^{\prime}-1\right)$. This strongly suggests that the instanton numbers of the pure $N=2$ Yang-Mills theory with gauge group $S U(k)$ are intimately related to those of SUSY QCD with $G=S U(2 k)$ and $N_{f}=2 k$. 


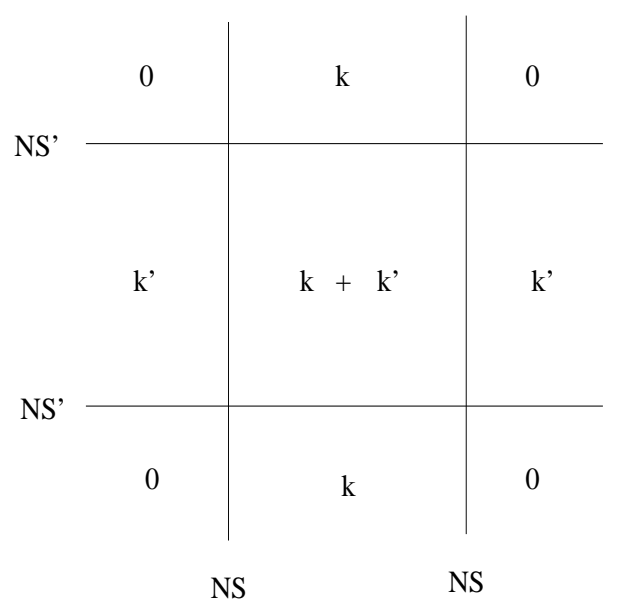

Fig. 4.6: Sewing of two pure $N=2$ Yang-Mills theories

At the end of this section we want extract the perturbative running of the $N=1$ gauge coupling constant. A priori we deal with two different Coulomb branches parametrised by $x^{3}+i x^{5}$ resp. by $x^{3}+i x^{7}$. In the following we will consider the common direction, $x^{3}$, and freeze the other directions, i.e. $x^{5}=x^{7}=0$. Now consider the box $\left[\alpha, \alpha^{\prime}\right]$ with the corresponding gauge group $S U\left(k_{\alpha}+k_{\alpha^{\prime}}^{\prime}\right)$. From eq. (4.19) we derive that

$$
\begin{aligned}
x_{\alpha^{\prime}+1}^{4}-x_{\alpha^{\prime}}^{4} & =L+\left(k_{\alpha^{\prime}+1}^{\prime}+k_{\alpha^{\prime}-1}^{\prime}-2 k_{\alpha^{\prime}}^{\prime}\right) \log x^{3}, \\
x_{\alpha+1}^{6}-x_{\alpha}^{6} & =L+\left(k_{\alpha+1}+k_{\alpha-1}-2 k_{\alpha}\right) \log x^{3},
\end{aligned}
$$

where $L$ is the classical distance between the NS5 and NS5'-branes. Then using eq. (4.4), the gauge coupling constants exhibits the following running behaviour:

$$
\begin{aligned}
\frac{1}{g_{\alpha, \alpha^{\prime}}^{2}} & =\left(g_{s}\right)^{-1}\left(L^{2}+L\left(k_{\alpha^{\prime}+1}^{\prime}+k_{\alpha^{\prime}-1}^{\prime}+k_{\alpha+1}+k_{\alpha-1}-2 k_{\alpha^{\prime}}^{\prime}-2 k_{\alpha}\right) \log x^{3}\right. \\
& \left.+\left(k_{\alpha^{\prime}+1}^{\prime}+k_{\alpha^{\prime}-1}^{\prime}-2 k_{\alpha^{\prime}}^{\prime}\right)\left(k_{\alpha+1}+k_{\alpha-1}-2 k_{\alpha}\right)\left(\log x^{3}\right)^{2}\right) .
\end{aligned}
$$

Since $N_{c}=k_{\alpha}+k_{\alpha^{\prime}}^{\prime}$ and $N_{f}=k_{\alpha^{\prime}+1}^{\prime}+k_{\alpha^{\prime}-1}^{\prime}+k_{\alpha^{\prime}}^{\prime}+k_{\alpha+1}+k_{\alpha-1}+k_{\alpha}$, the coefficient in front of $\log x^{3}$ precisely agrees with the one-loop $N=1 \beta$-function coefficient $b_{N=1}=-3 N_{c}+N_{f}{ }^{*}$.

\subsection{Concluding Remarks}

The example in Fig. 4.6 could be analysed in some more detail. From [89] one knows, that the number of deformations of the 3-cycle solution coincides with the first Betti

${ }^{*_{4}}$ It was already observed in $[10,88]$ that the brane box models with uniform bending lead to the correct $N=1$ $\beta$-function coefficients. 
number $b_{1}$ and if one pairs deformations into complex numbers \#moduli $=b_{1} / 2$. From the field theory side this should be related to the number of moduli of the gauge theory. The dimension of the moduli space of a $\mathrm{N}=2, \quad \mathrm{SU}\left(N_{c}\right)$ gauge theory in 3 dimensions with $N_{f}$ flavours can be obtained from the classical point of view by counting the number of scalar fields in the vector multiplet (Coulomb branch) and in the matter multiplets (Higgs branch), respectively. Classical there is an $N_{c}$ dimensional Coulomb branch and an $2 N_{f} N_{c}-N_{c}^{2}$ dimensional Higgs branch [86]. The quantum moduli spaces in $d=3, N=2$ were worked out in [86]. For $N_{f}=N_{c}$ the $N_{c}$-dimensional Coulomb branch will be lifted almost completely; only an 1-dim subbranch survives. The $N_{c}^{2}$-dimensional classical Higgs branch remains untouched. All in all one obtains for $N_{c}=N_{f}=4$ a moduli space of dimension:

$$
\operatorname{dim} \mathcal{M}=N_{c}^{2}+1=4^{2}+1=17 .
$$

Note the close connection of the 3 and 4 dimensional theories. In fact in [90] the same dimension is obtained for the genuine 4 dimensional theory.

This should be compared with the prediction derived from the special 3-cycle solution above. To that end we proceed to compute the first Betti number for the 3-cycle solution at hand. The both irreducible components of $\Sigma^{(3)}$ are given by the zero set of

$$
\begin{array}{ll}
\Sigma_{1} \times \mathbb{R}: & p_{1}=t^{2}+\left(v^{2}-u\right) \cdot t+1=0 \text { and } x^{7}=b_{2} \\
\Sigma_{2} \times \mathbb{R}: & p_{2}=\tilde{t}^{2}+\left(\tilde{v}^{2}-\tilde{u}\right) \cdot \tilde{t}+1=0 \text { and } x^{5}=b_{1}
\end{array}
$$

In the table below we point out the coordinates involved in the defining equations of the both 2-cycles $\Sigma_{1} \times \mathbb{R}_{4}$ and $\Sigma_{2} \times \mathbb{R}_{6}$. Here a " $\times$ " is filled in, if this coordinates appears

\begin{tabular}{|c|c|c|c|c|c|c|}
\hline & $\mathbb{R}_{3}$ & $\mathbb{R}_{4}$ & $\mathbb{R}_{5}$ & $\mathbb{R}_{6}$ & $\mathbb{R}_{7}$ & $\mathbb{R}_{10}$ \\
\hline$p_{1}$ & $\times$ & $\mathrm{L}$ & $\times$ & $\times$ & $b_{2}$ & $\times$ \\
$p_{2}$ & $\times$ & $\times$ & $b_{1}$ & $\mathrm{~L}$ & $\times$ & $\times$ \\
\hline & $?$ & $*$ & $b_{1}$ & $*$ & $b_{2}$ & $?$ \\
\hline
\end{tabular}

Tab. 4.4: Common intersection. in the defining equations $p_{1 / 2}$. The $b_{1 / 2}$ denote the coordinates held fixed on the irreducible component $p_{1 / 2}=0$. ' $\mathrm{L}$ ' is the line in $\Sigma \times \mathbb{R}$. In the last row we depict the generic result of the intersection of the two tori. "*" means there is always a common intersection, whereas "?" denotes the variables for which one has to decide the problem. The common intersection is given by:

$$
\begin{gathered}
p_{1}=t^{2}+\left(v^{2}-u\right) \cdot t+1=0 \quad \text { and } \quad p_{2}=\tilde{t}^{2}+\left(\tilde{v}^{2}-\tilde{u}\right) \cdot \tilde{t}+1=0 \\
x_{5}=b_{1}, \quad x_{7}=b_{2}, \quad x_{8}=0
\end{gathered}
$$

These are four real equations of third degree in four unknown variables and the generic solution are up to $\operatorname{deg}\left(p_{1}\right) \cdot \operatorname{deg}\left(p_{2}\right)=9$ points. Thus in the generic situation the two 
tori do intersect in points. Fig. 4.7 is a rough sketch of the situation in the case of touching points. It is a simple computation in simplicial homology to construct the matrix representation of the action of the boundary operator, which yields the complex below

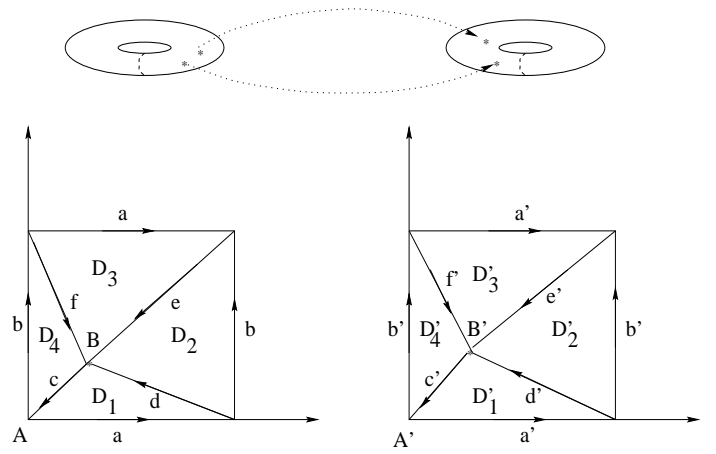

Fig. 4.7: 2-point unification of two tori.

$$
0 \rightarrow \mathbb{Z}_{2 \text {-cycles }}^{8} \stackrel{\partial_{2}}{\rightarrow} \mathbb{Z}_{1-\text { cycles }}^{12} \stackrel{\partial_{f}}{\rightarrow} \mathbb{Z}_{0 \text {-cycles }}^{2} \rightarrow 0
$$

Then we end up with

$$
H^{k}\left(T_{1}^{2} \cup_{2 \text { pts }} T_{2}^{2}\right)=\frac{\operatorname{ker} \partial_{k}}{\operatorname{im} \partial_{k+1}}=\left\{\begin{array}{lll}
\mathbb{Z}^{1} & : & k=0 \\
\mathbb{Z}^{5} & : & k=1 \\
\mathbb{Z}^{2} & : & k=2
\end{array}\right.
$$

Completely analogous one proceeds for the n-point unification so that the formula for the first Betti number is $b_{1}=4+n-1$. Here $n=\operatorname{deg}\left(p_{1}\right) \cdot \operatorname{deg}\left(p_{2}\right)=\left(N_{D 4}^{(1)}+1\right) \cdot\left(N_{D 4}^{(2)}+1\right)$, which is closely related to the numbers $N_{D 4}^{(1)}$ and $N_{D 4}^{(2)}$ of D4-branes in the polynomials $p_{1 / 2}$. If $N_{D 4}^{(1)}=N_{D 4}^{(2)}=N_{c} / 2=2$ then

$$
\text { \#moduli }=2+\frac{N_{c}^{2}}{8}+\frac{N_{c}}{2}=2+2+2 .
$$

What is the meaning of the number 6 ? The first 3 deformations are identified with the parameters appearing in the 2-cycle polynomials. For the other three the correct interpretation is still lacking. However it is possible to find this number in the relative positions of the branes again. Let us explain how this works ${ }^{*_{5}}$. In what follows we want to count the number of parameters for relative orientations of branes, which are compatible with the sewing ansatz, i.e. we want to maintain $N=2$ subsystems. Note that the branes (SLAG planes) of the $\mathrm{N}=2$ subsystems intersect pairwise in a line. To count moduli we start with a single brane. Each SLAG plane is generated by acting with an $S U(3)$-element on the real section of $\mathbb{C}^{3}$ (see Fig. C.2 in the appendix). We want to assume that the D5-branes of both subsystems are parallel. Thus we have to count the relative positions of two other SLAG planes which intersect the D5-brane in two lines, non parallel but with a common intersection. The relative position of the second line with respect to the first is described by two angles (the sphere in $\mathbb{R}^{3}$ ). Now restrict the attention to a second brane, which intersect the first in one of the two lines. The second plane is generated by acting with an element of $S U(2) \subset S U(3)$ on the two remaining directions. This contributes $\operatorname{dim} \mathcal{S L}(2)=\operatorname{dim} S U(2) / S O(2)=\mathbf{2}$ moduli. The last step is to put the third plane in the game, which intersect the first brane in the other line. We iterate the process and obtain again 2 moduli. To sum up we obtain

$$
2+2+2=6
$$

\footnotetext{
${ }^{*} 5$ We want to thank V. Braun for a discussion about this subject.
} 
real moduli describing brane positions in $\mathbb{R}^{6}$ compatible with the sewing ansatz. This should be the right interpretation for the other 3 complex parameters. 


\section{Chapter 5}

\section{Gauge Theory and Supergravity}

In this chapter we want to discuss a detail which comes out as a byproduct of the discussion in the last chapters. Here it becomes important, that we are concerned with the investigation of superconformal field theories (finite models). In a remarkable recent development it became clear that in a large class of superconformal field theories a new type of duality symmetry arises, namely they can be described equivalently by supergravity in Anti-de-Sitter (AdS) spaces [12]. In particular, there is a correspondence between four-dimensional superconformal field theories and supergravity on $A_{d} S_{5} \times M^{5}$, where $M^{5}$ is a certain five-dimensional Einstein space specified by the superconformal field theory we are concerned with. The examples discussed intensively in the literature are listed in Tab. 5.1:

\begin{tabular}{|c|c|c|c|}
\hline Einstein space: & $S^{5}$ & $T^{11}=\frac{S U(2) \times S U(2)}{U(1)}$ & def. $S^{5}$ \\
\hline Gauge theory: & $N=4 \mathrm{SYM}$ & D3-branes at conifold & $?$ \\
\hline
\end{tabular}

Tab. 5.1: Einstein spaces and CFT

In the simplest case, $M^{5}$ is given by $S^{5}$, and the corresponding superconformal field theory is just $N=4$ super Yang-Mills with $S U(n)$ gauge symmetry. This is precisely the superconformal theory which lives on the world volume of $n$ parallel D3-branes and slightly discussed in 1.3.1. Another well studied example for $M^{5}$ is the coset space $T^{1,1}$ which leads to a $N=1$ superconformal gauge theory, which is the superconformal theory of D3-branes probing the conifold singularity of eq. (2.3) [15].

The prescription of the holographic map [91, 92, 93, 94] allows for several non-trivial checks of the conjectured AdS/CFT correspondence. For example, the central charge 
of the conformal field theory is inversely proportional to the volume of $M^{5}$. This check works very nicely for the correspondence between the coset space $T^{1,1}$ and the superconformal field theory from D3-branes at the conifold singularity. Recently, deformations of the usual IIB string theory on $A d S_{5} \times S^{5}$ has been studied $[95,96,18]$ by analysing critical points of the scalar potential of $N=8$ gauged supergravity. $N=8$ gauged supergravity arises as a description for the effective theory of the massless modes in a $S^{5}$-Kaluza-Klein reduction. The Lagrangian of $N=8$ gauged supergravity was derived in [97]. In [18] a new critical point was found, leaving $N=2$ unbroken in the bulk (or $N=1$ on the brane), while preserving an $S U(2) \times U(1)$ subgroup of the $S O(6)$ gauged global symmetry. The information about this fixed point is completed by the ratio of the volume of the SUGRA solution corresponding to the $N=8$ critical point (dual to $N=4 \mathrm{SYM}$ - UV) to the volume of the SUGRA solution corresponding to the new critical point (dual to the unidentified $N=1$ theory - IR). The ratio yields

$$
\frac{V_{N_{8}}}{V_{N_{2}}}=\frac{c_{N_{1}}}{c_{N_{4}}}=\frac{27}{32} \text {. }
$$

The aim of this chapter is to show that this new fixed point corresponds to a particular mass deformation of the $N=4$ super Yang-Mills theory. It will be pointed out that the new fixed point field theories obtained by mass deforming the $N=4$ theory indeed reproduce the global symmetry as well as the ratio $c_{I R} / c_{U V}$ from the supergravity side. The labels "IR" and "UV" are taken from the applications in the context of renormalisation group flows [98].

In section 5.1 we will briefly explain the method [83] of deforming a supersymmetric field theory with a marginal operator to obtain a new class of superconformal models. Here it will be established that applying the same types of arguments to the deformation of the $N=4$ SUSY gauge theory by a mass term for one of the adjoint chiral multiplets one obtains a one parameter family of $N=1$ superconformal field theories. They can be expressed as $N=1$ theories with two massless adjoints $A$ and $B$ deformed by a quartic superpotential $W \sim(A B)^{2}$. This has to be contrasted with the mass deformation of the $N=4$ theories by a mass for a full hypermultiplet studied e.g. in [99]. While the latter one leads to a $N=2$ theory, this deformation leaves only $N=1$ unbroken.

In section 5.3 we will turn to the dual supergravity description provided by the supersymmetric fixed point found in [18] from the deformation of the $A d S_{5} \times S^{5}$ supergravity. While the field theory considerations presented up to this point are actually valid for an arbitrary gauge group, only the $S U(n)$ theories will be realized on D3-branes probes in IIB. 


\subsection{Construction of the New Conformal Theories}

Conformal field theories typically appear at the fixed points of the renormalisation group flow, where all couplings become unrenormalised with respect to the energy (inverse length) scale. If one starts from a given conformal field theory in $d=4$ dimensions one could deform it by adding additional terms $\mathcal{O}(x)$ to the action.

$$
S \rightarrow S+h \cdot \int d^{4} x \mathcal{O}(x)
$$

In general the deformed theory is not any longer a conformal one. To decide whether it is or not one has to take the scaling properties of $\mathcal{O}(x)$ into account. $\Delta$ denotes the scaling dimension of $\mathcal{O}(x)$. One can distinguish three cases in $d=4$ :

$$
\Delta=\left\{\begin{array}{ccc}
<4 & \text { relevant } & U V \stackrel{\text { strength }}{\stackrel{\longrightarrow}{\rightleftarrows} I R} \\
=4 & \text { marginal } & \text { no effect } \\
>4 & \text { irrel. } & U V \longleftarrow I R
\end{array}\right.
$$

Only marginal operators preserve the property to be a conformal theory. Last but not least a little complication arises. Quantum corrections may spoil the scaling properties of the field $\phi_{i}$ due to an anomalous dimension ${ }^{*_{1}} \gamma\left(\phi_{i}\right)=-\frac{\partial \ln Z_{i}}{\partial \ln \mu}$ it could pick up under renormalisation. Here $Z_{i}$ is the common $Z$-factor of the wave function renormalisation and $\mu$ the Wilsonian cutoff. For a certain field $\phi_{i}$ the scaling dimension $\Delta$ is then given as the sum of the canonical dimension $d\left(\phi_{i}\right)$ and the anomalous dimension $\gamma\left(\phi_{i}\right)$

$$
\Delta\left(\phi_{i}\right)=d\left(\phi_{i}\right)+\frac{1}{2} \gamma\left(\phi_{i}\right)
$$

Thus it is meaningless to talk about marginality without having control about the anomalous scaling behaviour. Operators $\mathcal{O}(x)$, which are protected against this unwanted feature are called exactly marginal operators. Actually it is quite hard to figure out which operator is a marginal one and which not, proving at the same time that one deforms inside the class of conformal theories.

The construction in [83] gives an indirect method to establish the existence of a family of finite $N=1$ theories through the construction of whole fixed manifolds. The basic idea is simply described. If one adds a perturbation $h \cdot \phi_{1} \ldots \phi_{n}$ with parameter $h$ to the action as in eq. (5.2) the authors of $[100,101]$ found the expression for the $\beta$-functions of the $h$-coupling:

$$
\beta_{h}=h(\mu)\left(-d_{W}+\sum_{k}\left[d\left(\phi_{k}\right)+\frac{1}{2} \gamma\left(\phi_{k}\right)\right]\right)=h(\mu) A_{h} .
$$

\footnotetext{
${ }^{* 1}$ The definition of anomalous dimension deviates from the standard one which absorbs the factor $1 / 2$.
} 
$d_{w}$ is the canonical dimension of the superpotential, which is 3 . Furthermore the $\beta$-function of the gauge coupling was computed in $[102,103]$ :

$$
\beta_{g}=-f(g[\mu])(\underbrace{\left[3 C_{2}(G)-\sum_{k} T\left(R_{k}\right)\right]}_{b_{0}}+\sum_{k} T\left(R_{k}\right) \gamma\left(\phi_{k}\right))=f(g[\mu]) A_{g} .
$$

Here $b_{0}$ is the 1-loop $\beta$-function coefficient and $T\left(R_{i}\right)$ is the Dynkin index of the representation $R_{i}$, which is defined by the trace on generators of the Lie algebra in the representation $R_{i}$ below:

$$
\operatorname{Tr}\left(T^{a} T^{b}\right)=T\left(R_{i}\right) \delta^{a b}
$$

$C_{2}(G)$ is the same for the fundamental representation.

At a fixed point all $\beta$-functions must vanish. Therefore the theories are in particular finite ones. In the case at hand the two coupling constant, will be determined from the two relations, eq. (5.3) and eq. (5.4). In general one could expect at most isolated solutions but if the equations are dependent a fixed line may appear. This simple observation will be applied to the special case of a mass deformed superconformal theory.

\subsection{The Flow from $N=4$ to $N=1$ Theories}

From the $N=1$ point of view, the field content of the $N=4$ theory is depicted in Tab. 5.2,

\begin{tabular}{|c|c|c|}
\hline$A_{\mu}$ & $4 \lambda_{\alpha A}$ & 6 real $\Phi^{I}$ \\
& in $\overline{4}$ of $S U_{R}(4)$ & in 6 of $S U_{R}(4)$ \\
\hline
\end{tabular}

Tab. 5.2: $\mathrm{N}=4$ Vector multiplet

which provides us with three chiral matter fields $\Phi_{a}$ in the adjoint representation of the gauge group. In $N=1$ terms the $S U(4)$ R-symmetry is not manifest any longer but the chiral fields (the six real scalars combine into the three complex scalar components) transforms under the residual R-symmetry group

$$
\Phi_{a} \in 3_{\frac{2}{3}} \text { of } S U(3) \times U_{R}(1) \in S U_{R}(4)
$$


The superpotential of eq. (1.31) is a marginal operator of the $N=4$ theory,

$$
W=g f^{a b c} \Phi_{a} \Phi_{b} \Phi_{c}
$$

which after appropriate renaming of the fields reads:

$$
W=g f^{a b c} A_{a} B_{b} X_{c} .
$$

Now we add the mass term

$$
W_{\text {mass }}=m\left(X_{c}\right)^{2}
$$

for the chiral field $X_{c}$. This mass term breaks the original R-symmetry and the fields get the new R-charges as shown in Tab. 5.3.

\begin{tabular}{|c|c|c|c|}
\hline field & $A_{a}$ & $B_{b}$ & $X_{c}$ \\
\hline$r$ & $1 / 2$ & $1 / 2$ & 1 \\
\hline
\end{tabular}

Tab. 5.3: R-charges of the chiral superfields

For the effective theory of massless modes one should integrate out the heavy field which simply amounts to use the equation of motion to eliminate the field $X_{c}$ from the superpotential

$$
\frac{\partial W}{\partial X_{c}}=g f^{a b c} A_{a} B_{b} \stackrel{!}{=} 2 m X_{c}
$$

As a result we find that any $N=1$ theory with two adjoint matter fields $A$ and $B$ allows for a marginal deformation by adding the quartic superpotential

$$
W=h \cdot f^{a b c} f^{d e c} A_{a} B_{b} A_{d} B_{e} \quad h=\frac{g^{2}}{2 m} .
$$

That this is in fact a marginal operator follow from the formulas in eq. (5.3) and eq. (5.4). At first all matter fields are in the same representation and one obtains $C_{2}(G)=T\left(R_{i}\right)$. A second piece of information originates from the residual R-symmetry. The surviving $S U(2)$ acts on the fields $A$ and $B$ and put them into doublets. Therefore the anomalous 
dimensions of these fields coincide. Having this in mind, we write out the equations (5.3) and (5.4):

$$
\begin{array}{ll}
\beta_{g} \sim 3-2+2 \cdot \gamma(g, h) \stackrel{!}{=} 0 \\
\beta_{h} \sim 1+2 \cdot \gamma(g, h) \stackrel{!}{=} 0
\end{array}
$$

These equations are in fact dependent giving rise to a fixed line of conformal field theories and one can convince oneself that the new superpotential really has the right scaling property $(\Delta=4)$.

Note that not all values of this marginal coupling are distinct. There exists an S-duality inherited from the $N=4$ theory, mapping strong coupling to weak coupling. Looking at the special point where the quartic superpotential is turned off, we find the interesting conclusion that all $N=1$ theories with 2 adjoints are self dual under Seiberg duality in the sense that electric and magnetic theory have the same gauge group, but the magnetic theory has in addition a cubic superpotential coupling to some singlet meson fields.

Let us add some comments about this model:

1. This deformation is not the same as the deformation of the $N=4$ theory by a mass term for a full hypermultiplet as e.g. studied in [99]. The latter leaves an $N=2$ SUSY unbroken and mass deformation is given by one complex parameter. In our case it is just one real mass parameter.

2. A very similar family of $N=1$ superconformal theories exists, which arise as mass deformed $N=2$ theories. Deforming the finite $N=2 S U(n)$ theory with $2 n$ flavours by a mass for the adjoint chiral multiplet leaves an $N=1$ theory with a quartic superpotential. The superconformal theory along the fixed line parametrised by the marginal operator is precisely the superconformal theory of $n$ D3-branes probing a conifold.

\subsection{The dual Supergravity Description}

Now we come back to the point we started from in the foreword to this chapter. Following the general idea of [12] one would expect that these conformal field theories do have a dual supergravity description. Since the field theory constructed in subsection 5.2 arises as a mass deformation of $N=4 \mathrm{SYM}$, the dual supergravity description should be a deformation of the usual IIB string theory on $A d S_{5} \times S^{5}$. In $[95,96,18]$ such deformations where studied by analysing critical points of $N=8$ gauged supergravity. In 
[18] a new fixed point was found, leaving $N=2$ unbroken in the bulk (corresponding to $N=1$ on the brane). We will argue that this deformation indeed corresponds to the dual of the superconformal field theories we were studying in this chapter.

As a first piece of evidence for having correctly assigned the SUGRA solution of [18] to the conformal $N=1$ theory obtained by a mass deformation of $N=4$ super Yang-Mills theory let us compare the global symmetries. According to [18] the subgroup of $S O(6)$ unbroken by the solution is $S U(2) \times U(1)$. The $S U(2)$ in the field theory rotates the 2 adjoints into each other. The $A B A B$ super potential is invariant. The $U(1)$ is the $U(1)_{R}$ symmetry of the $N=1$ theory under which $A$ and $B$ both have charge $1 / 2$.

A more quantitative test is to compare the ratio of central charges $c$ of the deformed (IR) and undeformed (UV) theories. On the supergravity side the ratio was computed and the prediction is $27 / 32$. Let us show that this value is reproduced by the proposed dual field theory. The computation follows [94].

The quotient of the central charges of the IR and UV conformal theories can be calculated using a trick. The central charge $c$ appears in the formula of the Weyl anomaly, which has the general structure [104]:

$<T_{\mu}^{\mu}>_{g_{\mu \nu}, V_{\mu}}=a \cdot\left[-\frac{1}{16 \pi^{2}} \tilde{R}_{\mu \nu \rho \sigma} \tilde{R}^{\mu \nu \rho \sigma}\right]+c \cdot\left[\frac{1}{16 \pi^{2}} \tilde{W}_{\mu \nu \rho \sigma} \tilde{W}^{\mu \nu \rho \sigma}+\frac{1}{6 \pi^{2}} V_{\mu \nu} V^{\mu \nu}\right]$

Here $a$ is the axial charge, the subscripts $g_{\mu \nu}$ and $V_{\mu}$ are the sources of $T_{\mu \nu}$ and $R_{\mu}$ in the generating functional and $\tilde{R}_{\mu \nu \rho \sigma}$ is the dual of the Riemannian curvature $R_{\mu \nu \rho \sigma}$. A useful property of $N=1$ super conformal algebras is the fact that the currents fit into a supermultiplet of currents, which determines on the other side a supermultiplet of anomalies. Therefore the Weyl anomaly and the anomaly of the R-charge are not independent. In fact the latter is given by:

$$
<\partial_{\mu} \sqrt{g} R^{\mu}>_{g_{\mu \nu}, V_{\mu}}=-\frac{a-c}{24 \pi^{2}} R_{\mu \nu \rho \sigma} \tilde{R}^{\mu \nu \rho \sigma}+\frac{5 a-3 c}{9 \pi^{2}} V_{\mu \nu} \tilde{V}^{\mu \nu}
$$

The important point to note is the appearance of the constants $a$ and $c$ we are interested in in the latter formula. Thus one can determine the Weyl anomaly from the anomaly of the R-charge, which is a rather standard computation in quantum field theory.

Taking appropriate variations of eq. (5.7) with respect to the sources $g_{\mu \nu}$ and $V_{\mu}$ respectively, one obtains two relations: 


$$
\begin{aligned}
a-c & \sim<\partial_{\mu} \sqrt{g} R^{\mu} T_{\alpha \beta} T_{\gamma \delta}>, \\
5 a-3 c & \sim<\partial_{\mu} \sqrt{g} R^{\mu} R_{\alpha} R_{\beta}>.
\end{aligned}
$$

The constants of proportionality does not really matter as we take quotients later on and they drop out.

The correlators of eq. (5.8) can be computed from the famous triangle graphs (see Fig. 5.1) below.
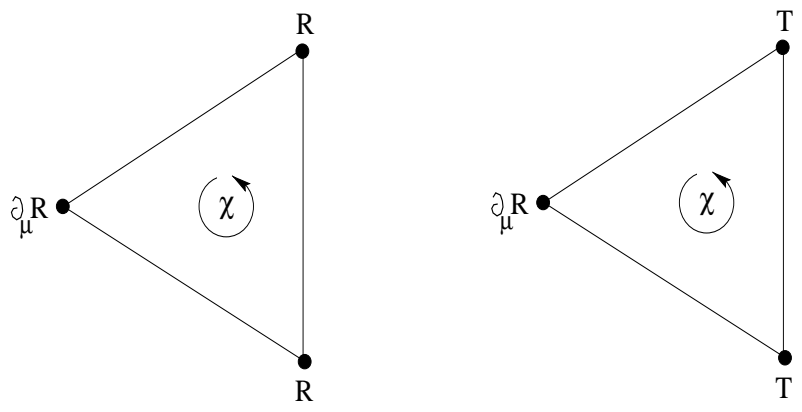

Fig. 5.1: Triangle graphs

The pleasant property of this types of graphs is that a particle couples via its R-charge to each of the R-current vertices. Therefore the graph on the left hand side is proportional to the sum of third powers of R-charges of each propagating particle in the loop while the graph on the right hand side does the same but only to the first power of R-charges.

UV - N = 4 SYM: First consider the UV theory. We have $\left(N_{c}^{2}-1\right)$ gauginos with $r=1$ and $3 \cdot\left(N_{c}^{2}-1\right)$ matter fermions with $r=-1 / 3$ (the superpotential has to have $r=2$, so the scalars have $r=2 / 3$ and the fermions $r=-1 / 3)$.

\begin{tabular}{|c|r|}
\hline$\chi$ & $r(\chi)$ \\
\hline$N_{c}^{2}-1 \quad$ gauginos & 1 \\
$\left(N_{c}^{2}-1\right) \cdot 3$ matter fermions & $-\frac{1}{3}$ \\
\hline
\end{tabular}

Tab. 5.4: R-charges of $N=4$ 
$<\partial_{\mu} \sqrt{g} R T T>$ is given by the sum of all R-charges,

$$
a-c \sim \sum r(\chi)=0
$$

hence $a=c$. Moreover

$$
5 a-3 c \sim \sum r^{3}(\chi)=\left(N_{c}^{2}-1\right) \cdot 1+3\left(N_{c}^{2}-1\right) \cdot\left(-\frac{1}{3}\right)^{3}=\frac{8}{9}\left(N_{c}^{2}-1\right)
$$

and therefore $c \sim \frac{4}{9}\left(N_{c}^{2}-1\right)$.

IR - deformed N = 4 SYM: In the IR we see the mass deformed $N=1$ theory with a superpotential given in eq. (5.6). So we are left with $\left(N_{c}^{2}-1\right)$ gauginos with R-charge $r=1$ accompanied by $2 \cdot\left(N_{c}^{2}-1\right)$ matter fermions $A, B$ with R-charge $r=-1 / 2$.

\begin{tabular}{|c|r|}
\hline$\chi$ & $r(\chi)$ \\
\hline$N_{c}^{2}-1 \quad$ gauginos & 1 \\
$\left(N_{c}^{2}-1\right) \cdot 2$ matter fermions & $-\frac{1}{2}$ \\
\hline
\end{tabular}

Tab. 5.5: R-charges of def. $N=4$

Therefore

$$
\begin{aligned}
a-c & \sim \sum r(\chi)=0 \\
5 a-3 c & \sim \sum r^{3}(\chi)=\left(N_{c}^{2}-1\right) \cdot 1+2\left(N_{c}^{2}-1\right) \cdot\left(-\frac{1}{2}\right)^{3}=\frac{3}{4}\left(N_{c}^{2}-1\right)
\end{aligned}
$$

and comparing finally with the supergravity prediction mentioned above one finds

$$
\frac{c_{I R}}{c_{U V}}=\frac{\frac{3}{8}}{\frac{4}{9}}=\frac{27}{32}
$$

in complete agreement. Finally one can determine the absolute value of the central charge from the known value of $c_{U V}$. The UV theory is the unbroken $N=4$ theory. 
Thus the central charge will be given just by the free field contributions and adds up to $c=1 / 4 \cdot\left(N_{c}^{2}-1\right)$. Then the central charge of the interacting IR theory is given by

$$
c_{I R}=\frac{27}{128} \cdot\left(N_{c}^{2}-1\right)
$$

Note that the numerical value $27 / 32$ is precisely the same as the one obtained in the related setup of the conifold as viewed as a mass deformation of the $\mathbb{Z}_{2}$ orbifold [94, 2]. This lead [18] to the speculation that these two theories are indeed related. Here we see that they are quite distinct. The reason for the matching of the numerical values is just due to the mechanism by which we deform: a finite theory with a cubic superpotential (the only choice in a finite theory) is deformed by a mass term, giving rise to quartic superpotential while killing $1 / 3$ of the fields. The superpotential uniquely fixes the R-charge which in turn determines the central charge. 


\section{Appendix A}

\section{Definitions}

Metric and $\varepsilon$-Symbol

$$
\begin{gathered}
\eta_{a b}=\operatorname{diag}(-1,1 \ldots 1) \\
\epsilon^{01 \ldots n-1 n}=1 \\
\epsilon_{a_{0} \ldots a_{n}}=g \epsilon^{a_{0} \ldots a_{n}} \\
\epsilon^{a_{0} \ldots a_{n}} \epsilon_{b_{0} \ldots b_{n}}=g \delta_{b_{0} \ldots b_{n}}^{a_{0} \ldots a_{n}} \\
\epsilon^{a_{0} \ldots a_{i} a_{i+1} \ldots a_{n}} \epsilon_{b_{0} \ldots b_{i} a_{i+1} \ldots a_{n}}=g \delta_{b_{0} \ldots b_{i} a_{i+1} \ldots a_{n}}^{a_{0} \ldots a_{n}}=g(n-i) ! \cdot \delta_{b_{0} \ldots b_{i}}^{a_{0} \ldots a_{i}} \\
\epsilon^{a_{0} \ldots a_{n}} \epsilon_{a_{0} \ldots a_{n}}=g \cdot n !
\end{gathered}
$$

\section{Vielbein}

The change from spacetime components to tangent frame components can be performed via the vielbein $e^{a}{ }_{\mu}\left(e^{a}=e^{a}{ }_{\mu} d x^{\mu}\right)$,

$$
e_{\mu}^{a}=(\stackrel{\stackrel{\mu}{\longrightarrow}}{a \downarrow})
$$

which satisfies

$$
\eta_{a b} e^{a}{ }_{\mu} e^{b}{ }_{\nu}=g_{\mu \nu} \quad g^{\mu \nu} e^{a}{ }_{\mu} e^{b}{ }_{\nu}=\eta^{a b}
$$


and is given by

The inverse operation would be performed by the "inverse" vielbein $\tilde{e}^{\mu}{ }_{b}$, which is defined by:

$$
e^{a}=e^{a}{ }_{\mu} d x^{\mu}=e^{a}{ }_{\mu} \tilde{e}^{\mu}{ }_{b} e^{b} \Rightarrow e^{a}{ }_{\mu} \tilde{e}^{\mu}{ }_{b}=\delta_{b}^{a} \text { and } \tilde{e}^{\mu}{ }_{a} e^{a}{ }_{\nu}=\delta_{\nu}^{\mu}
$$

Obviously it is precisely the inverse matrix of $e^{a}{ }_{\mu}$. Finally

$$
e_{a}{ }^{\mu}=\left(\tilde{e}^{\mu}{ }_{a}\right)^{\dagger} \quad \tilde{e}_{\mu}{ }^{a}=\left(e^{a}{ }_{\mu}\right)^{\dagger}
$$

\section{Gamma Matrices}

The $S O(1,10)$ vector index $a$ splits under the residual $S O(1,5) \times S O(5)$ group into $a=\left(a, a^{\prime}\right)$, which corresponds to the space-time directions tangential or normal to the brane, respectively.

What are the corresponding spin groups?

$$
\begin{array}{rll}
\operatorname{Spin}(1,5)=S U^{*}(4) & \alpha=1 \ldots 4 \\
\operatorname{Spin}(5)=U S p(4) & i=1 \ldots 4 \\
& \\
0 \longrightarrow \operatorname{Spin}(1,5) \times \operatorname{Spin}(5) & \longrightarrow & \operatorname{Spin}(1,10) \longrightarrow 0 \\
\left(\psi^{\alpha} \oplus \bar{\psi}_{\dot{\alpha}}, \chi^{i}\right) & \stackrel{\otimes}{\mapsto} & \psi^{\alpha i} \oplus \bar{\psi}_{\dot{\alpha}}^{i}
\end{array}
$$

5-dimensional Euclidean Clifford algebra $\mathcal{C} \ell(0,5): \quad g_{i j}=\delta_{i j}$

$$
\left\{\gamma^{i}, \gamma^{j}\right\}=2 \delta^{i j} \quad i, j=1, \ldots, 5
$$

6-dimensional Minkowskian Clifford algebra $\mathcal{C} \ell(1,5): \quad \eta_{a b}=\operatorname{diag}(-1,1, \ldots, 1)$

We want to define a 6-dimensional Minkowskian Clifford algebra in terms of the 5dimensional Euclidean Clifford algebra given before. Of course we need an additional 
generator of the underlying vector space. Furthermore the general theory of Clifford algebras teaches, that we can not prevent a doubling of the dimension of the matrices in going from a five to a six dimensional one. We will get an satisfying answer after considering some preliminary facts. Choose

$$
\gamma^{0}=-\tilde{\gamma}^{0}=\mathbb{1} \text { and } \quad \tilde{\gamma}^{i}=\gamma^{i}
$$

then

$$
\begin{aligned}
\gamma^{0}\left(-\gamma^{0}\right)+\gamma^{0}\left(-\gamma^{0}\right) & =2 \eta^{00} \mathbb{1} \\
\gamma^{0} \tilde{\gamma}^{0}+\gamma^{0} \tilde{\gamma}^{0} & =2 \eta^{00} \mathbb{1}
\end{aligned}
$$

On the other side for $i=1, \ldots, 5$ :

$$
\gamma^{0} \tilde{\gamma}^{i}+\gamma^{i} \tilde{\gamma}^{0}=0
$$

So we can combine the last formula and the 5-dimensional Euclidean Clifford algebra into a new compact definition $a=0, \ldots, 5$ :

$$
\gamma^{a} \tilde{\gamma}^{b}+\gamma^{b} \tilde{\gamma}^{a}=2 \eta^{a b} \mathbb{1}
$$

But now we can define the 6-dimensional Minkowskian Clifford algebra by

$$
\left(\tilde{\Gamma}^{a}\right)_{\alpha}{ }^{\beta}=\left(\begin{array}{cc}
0 & \left(\gamma^{a}\right)_{\alpha \beta} \\
\left(\tilde{\gamma}^{a}\right)^{\alpha \beta} & 0
\end{array}\right) \quad a=0, \ldots, 5
$$

which in fact satisfy

$$
\left\{\tilde{\Gamma}^{a}, \tilde{\Gamma}^{b}\right\}=2 \eta^{a b} \quad a, b=0, \ldots, 5 .
$$

The linear independence of the generators of the underlying vector space is ensured by the relative minus sign in front of the $\gamma^{0}$ and $\tilde{\gamma}^{0}$ matrices, respectively. Since $\gamma^{i}$ are linear independent, the both equations $\pm \mathbb{1}=\sum c_{i} \gamma^{i}$ cannot be fulfilled simultaneously. 
A general property:

Let $\lambda=e_{1} \cdot \ldots \cdot e_{k+l}$ be the chirality operator of an even dimensional Clifford algebra $\mathcal{C} \ell(k, l)$ of signature $(k, l)$. Then

$$
\kappa=\lambda^{2}=(-1)^{\frac{(k-l-1)(k-l)}{2}}
$$

Let $V$ be the even dimensional vector space underlying the even dimensional Clifford algebra mentioned before. Then there is a Clifford map $j$, which establishes the graded isomorphism below:

$$
\begin{aligned}
\mathcal{C} \ell(V \oplus W, \eta \oplus \kappa g) & \stackrel{\cong}{\longrightarrow} \mathcal{C} \ell(V, \eta) \otimes \mathcal{C} \ell(W, g) \\
j(v \oplus w) & =v \otimes \mathbb{1}+\lambda \otimes w
\end{aligned}
$$

Specialising to the case at hand, we obtain:

$$
\begin{aligned}
\mathcal{C} \ell\left(\mathbb{R}^{1,5} \oplus \mathbb{R}^{5}, \eta \oplus g\right) & \stackrel{\cong}{\longrightarrow} \mathcal{C} \ell\left(\mathbb{R}^{1,5}, \eta\right) \otimes \mathcal{C} \ell\left(\mathbb{R}^{5}, g\right) \\
j(v \oplus w) & =v \otimes \mathbb{1}+\lambda \otimes w
\end{aligned}
$$

$\underline{\text { 11-dimensional Clifford algebra } \mathcal{C} \ell(1,10):}$

$$
\begin{aligned}
& \left(\Gamma^{a}\right)_{\underline{\alpha}} \underline{\beta}=\delta_{i}^{j}\left(\begin{array}{cc}
0 & \left(\gamma^{a}\right)_{\alpha \beta} \\
\left(\tilde{\gamma}^{a}\right)^{\alpha \beta} & 0
\end{array}\right) \quad\left(\Gamma^{a^{\prime}}\right)_{\underline{\alpha}} \underline{\beta}=\left(\gamma^{a^{\prime}}\right)_{i}{ }^{j}\left(\begin{array}{cc}
\delta_{\alpha}{ }^{\beta} & 0 \\
0 & -\delta^{\alpha}{ }_{\beta}
\end{array}\right) \\
& \left\{\Gamma^{a}, \Gamma^{b}\right\}=2 \eta^{a b} \delta_{i}^{j}\left(\begin{array}{cc}
\delta_{\alpha}{ }^{\beta} & 0 \\
0 & \delta^{\alpha}{ }_{\beta}
\end{array}\right), \quad\left\{\Gamma^{a^{\prime}}, \Gamma^{b^{\prime}}\right\}=2 \delta^{a^{\prime} b^{\prime}} \delta_{i}^{j}\left(\begin{array}{cc}
\delta_{\alpha}{ }^{\beta} & 0 \\
0 & \delta^{\alpha}{ }_{\beta}
\end{array}\right), \quad\left\{\Gamma^{a}, \Gamma^{a^{\prime}}\right\}=0 . \\
& \left(\Gamma^{a_{1} \ldots a_{2 n} b_{1}^{\prime} \ldots b_{m}^{\prime}}\right)_{\underline{\alpha}} \underline{\underline{\beta}}=\left(\gamma^{b_{1}^{\prime} \ldots b_{m}^{\prime}}\right)_{i}{ }^{j}\left(\begin{array}{cc}
\left(\gamma^{a_{1} \ldots a_{2 n}}\right)_{\alpha}{ }^{\beta} & 0 \\
0 & (-1)^{m}\left(\tilde{\gamma}^{a_{1} \ldots a_{2 n}}\right)^{\alpha}{ }_{\beta}
\end{array}\right) \\
& \left(\Gamma^{a_{1} \ldots a_{2 n+1} b_{1}^{\prime} \ldots b_{m}^{\prime}}\right)_{\underline{\alpha}} \underline{\underline{\beta}}=\left(\gamma^{b_{1}^{\prime} \ldots b_{m}^{\prime}}\right)_{i}{ }^{j}\left(\begin{array}{cc}
0 & (-1)^{m}\left(\gamma^{a_{1} \ldots a_{2 n+1}}\right)_{\alpha \beta} \\
\left(\tilde{\gamma}^{a_{1} \ldots a_{2 n+1}}\right)^{\alpha \beta} & 0
\end{array}\right)
\end{aligned}
$$




$$
\left.\gamma^{a_{1} \ldots a_{n}}=\gamma^{\left[a_{1}\right.} \tilde{\gamma}^{a_{2}} \gamma^{a_{3}} \ldots{ }^{a_{n}}\right]
$$

\section{Duality relation:}

We need a specialisation of the duality relation for 6-dimensional Gamma matrices given below.

$$
\begin{aligned}
& \tilde{\Gamma}^{a_{1} \ldots a_{n}}=\frac{1}{(6-n) !}(-1)^{\frac{n(n+1)}{2}} \epsilon^{a_{1} \ldots a_{n} a_{n+1} \ldots a_{6}} \tilde{\Gamma}_{0 \ldots 5} \tilde{\Gamma}_{a_{n+1} \ldots a_{6}} \\
& =-\frac{1}{(6-n) !}(-1)^{\frac{n(n+1)}{2}} \epsilon_{a_{1} \ldots a_{n} a_{n+1} \ldots a_{6}} \tilde{\Gamma}_{0 \ldots 5} \tilde{\Gamma}_{a_{n+1} \ldots a_{6}} \\
& =+\frac{1}{(6-n) !}(-1)^{\frac{n(n+1)}{2}} \tilde{\Gamma}_{a_{1} \ldots a_{n} a_{n+1} \ldots a_{6}} \tilde{\Gamma}_{a_{n+1} \ldots a_{6}} \\
& =+\frac{1}{(6-n) !}(-1)^{\frac{n(n+1)}{2}} \tilde{\Gamma}_{a_{1} \ldots a_{n} a_{n+1}^{(0)} \ldots a_{6}^{(0)}} \underbrace{\tilde{\Gamma}_{a_{n+1}^{(0)} \ldots a_{6}^{(0)}}}_{\text {fix.Belegung }} \cdot(6-n) ! \\
& =+(-1)^{\frac{n(n+1)}{2}} \tilde{\Gamma}_{a_{1} \ldots a_{n}} \tilde{\Gamma}_{a_{n+1}^{(0)}} \cdots \tilde{\Gamma}_{a_{6}^{(0)}} \tilde{\Gamma}_{a_{n+1}^{(0)}} \cdots \tilde{\Gamma}_{a_{6}^{(0)}} \\
& =+(-1)^{\frac{n(n+1)}{2}} \tilde{\Gamma}_{a_{1} \ldots a_{n}} \cdot \underbrace{(-1)^{(6-n-1)+\ldots+1}}_{(-1)^{\frac{n(n+1)}{2}+1}} \cdot \tilde{\Gamma}_{a_{n+1}^{(0)}}^{2} \cdots \tilde{\Gamma}_{a_{6}^{(0)}}^{2} \\
& =-\tilde{\Gamma}_{a_{1} \ldots a_{n}} \cdot \tilde{\Gamma}_{a_{n+1}^{(0)}}^{2} \cdots \tilde{\Gamma}_{a_{6}^{(0)}}^{2}=\tilde{\Gamma}^{a_{1} \ldots a_{n}}
\end{aligned}
$$

Using the explicit form of the antisymmetric product of Gamma matrices we can derive the following lines for even $n$ :

$$
\begin{aligned}
\tilde{\Gamma}^{a_{1} \ldots a_{n}}= & \frac{1}{(6-n) !}(-1)^{\frac{n(n+1)}{2}} \epsilon^{a_{1} \ldots a_{n} a_{n}+1 \ldots a_{6}} \tilde{\Gamma}_{0 \ldots 5} \tilde{\Gamma}_{a_{n+1} \ldots a_{6}} \\
= & \frac{1}{(6-n) !}(-1)^{\frac{n(n+1)}{2}} \epsilon^{a_{1} \ldots a_{n} a_{n+1} \ldots a_{6}} . \\
& \left(\begin{array}{cc}
\mathbf{1} & 0 \\
0 & -\mathbb{1}
\end{array}\right) \cdot\left(\begin{array}{cc}
\left(\gamma_{\left.a_{n+1} \ldots a_{6}\right)_{\alpha} \beta}\right. & 0 \\
0 & \left(\tilde{\gamma}_{a_{n+1} \ldots a_{6}}\right)^{\alpha}{ }_{\beta}
\end{array}\right)
\end{aligned}
$$

A completely similar computation holds for $n$ odd. Then for all $n$ we find the same duality relation

$$
\gamma^{a_{1} \ldots a_{n}}=\frac{1}{(6-n) !}(-1)^{\frac{n(n+1)}{2}} \epsilon^{a_{1} \ldots a_{n} a_{n+1} \ldots a_{6}} \gamma_{a_{n+1} \ldots a_{6}}
$$


or with an extra minus sign for $\tilde{\gamma}^{a_{1} \ldots a_{n}}$. 


\section{Appendix B}

\section{Toric Data of Singularities}

\section{Orbifold Geometries}

The (complex) 3-dimensional orbifold geometries we are studying are

- The flat complex space $\mathbb{C}^{3}$ with coordinates $z_{1}=x^{4}+i x^{5}, z_{2}=x^{6}+i x^{7}$ and $z_{3}=$ $x^{8}+i x^{9}$.

- The $\mathbb{Z}_{k} \times \mathbb{Z}_{l}$ orbifold $\mathcal{O}_{k l}=\mathbb{C}^{3} /\left(\mathbb{Z}_{k} \times \mathbb{Z}_{l}\right)$, being defined as

\begin{tabular}{|rrr|rrr|}
\hline \multicolumn{2}{|c|}{$\mathbb{Z}_{k}$} & \multicolumn{3}{c|}{$\mathbb{Z}_{l}$} \\
\hline$z_{1}$ & $\mapsto$ & $e^{-2 \pi i / k} \cdot z_{1}$ & $z_{1}$ & $\mapsto$ & $e^{-2 \pi i / l} \cdot z_{1}$ \\
$z_{2}$ & $\mapsto$ & $z_{2}$ & $z_{2}$ & $\mapsto$ & $e^{2 \pi i / l} \cdot z_{2}$ \\
$z_{3}$ & $\mapsto$ & $e^{2 \pi i / k} \cdot z_{3}$ & $z_{3}$ & $\mapsto$ & \\
\hline
\end{tabular}

The defining equations of the orbifold are given by writing down the relations between the invariant monomials which are

\begin{tabular}{|c|cccccc|}
\hline & $u_{1}$ & $u_{2}$ & $u_{3}$ & $u_{4}$ & $u_{5}$ & $u_{6}$ \\
\hline inv. monomials & $z_{1}^{k l}$ & $\left(z_{1} \cdot z_{3}\right)^{k}$ & $z_{3}^{k}$ & $\left(z_{1} \cdot z_{2}\right)^{l}$ & $z_{2}^{l}$ & $z_{1} z_{2} z_{3}$ \\
\hline
\end{tabular}

By the map, which associates invariant polynomials with lattice points

$$
M: z_{1}^{a} z_{2}^{b} z_{3}^{c} \mapsto(a, b, c)^{T}
$$

we get a list of generating elements of the lattice of invariant monomials as the columns of $M$. Since obviously the lattice is at most three dimensional, on the other hand there 
are more than three generating elements, we expect relations in the $u_{i}$. To compute these relations we have do deal with integral lattices. A relation is an element of the preimage of $M$ which gets map to zero in the image of $M$, i.e. a certain sum of generating vectors of the lattice combine to zero. But this is certainly the kernel of the map mentioned before. The matrix representation of $M$ is simply

$$
M=\left(\begin{array}{cccccc}
k l & k & 0 & l & 0 & 1 \\
0 & 0 & 0 & l & l & 1 \\
0 & k & k & 0 & 0 & 1
\end{array}\right)
$$

Then the kernel is given by

$$
\operatorname{ker} M=\operatorname{span}\{(1,-l, 0,-k, 0, k l),(0,0, l, k, 0,-k l),(0, l, 0,0, k,-k l)\}
$$

If one translate these relations back one obtains the equations, which describe this special singularity:

$$
u_{1} \cdot u_{6}^{k l}=u_{2}^{l} u_{4}^{k} \quad u_{3}^{l} \cdot u_{4}^{k}=u_{6}^{k l} \quad u_{2}^{l} \cdot u_{5}^{k}=u_{6}^{k l} .
$$

Again we can best encode the possible blowups in a toric language. The corresponding diagram was shown in Fig.2.3. One can view this geometry as a partial blowup of the orbifolded conifold, which was given by the toric diagram in Fig.2.4. Basically by sending all $m_{i j}$ in the upper triangle to infinity we "remove" half of the two-cycles, leaving us with the orbifold geometry.

\section{Orbifolded Conifold}

The defining equations of the conifold can be obtained by writing down the relations between the invariant monomials which are

\begin{tabular}{|l|cccccc|}
\hline & $u_{1}$ & $u_{2}$ & $u_{3}$ & $u_{4}$ & $u_{5}$ & $u_{6}$ \\
\hline inv. monomials & $x^{l}$ & $y^{l}$ & $x y$ & $u^{k}$ & $v^{k}$ & $u v$ \\
\hline
\end{tabular}

By the map, which associates invariant polynomials with lattice points

$$
M: \quad x^{a} y^{b} u^{c} v^{d} \mapsto(a, b, c, d)^{T}
$$

we get a list of generating elements of the lattice $\mathbb{M}$ of invariant monomials as the columns of $M$. Since obviously the lattice is at most four dimensional, on the other hand there are more than four generating elements, we expect relations in the $u_{i}$. A relation is an element of the preimage of $M$ which gets map to zero in the image of $M$, i.e. a certain 
sum of generating vectors of the lattice add up to zero. But this is certainly the kernel of the map mentioned before. The matrix representation of $M$ is simply

$$
M=\left(\begin{array}{llllll}
l & 0 & 1 & 0 & 0 & 0 \\
0 & l & 1 & 0 & 0 & 0 \\
0 & 0 & 0 & k & 0 & 1 \\
0 & 0 & 0 & 0 & k & 1
\end{array}\right)
$$

Then the kernel is given by

$$
\operatorname{ker} M=\operatorname{span}\{(1,1,-l, 0,0,0),(0,0,0,1,1,-k)\}
$$

If we translate these relations back to equations we obtain

$$
u_{1} \cdot u_{2}=u_{3}^{l} \quad \text { and } \quad u_{4} \cdot u_{5}=u_{6}^{k}
$$

With the help of the conifold equation $x y-u v=u_{3}-u_{6}$ one can eliminate a further variable, such that the resulting equations read

$$
u_{1} \cdot u_{2}=u_{3}^{l} \quad \text { and } \quad u_{4} \cdot u_{5}=u_{3}^{k}
$$

Renaming the variables to more conventional ones $\left(x^{\prime}=u_{1}, y^{\prime}=u_{2}, z=u_{3}, u^{\prime}=u_{4}\right.$, $\left.v^{\prime}=u_{5}\right)$ we obtain

$$
\begin{aligned}
& x^{\prime} y^{\prime}=z^{l} \\
& u^{\prime} v^{\prime}=z^{k}
\end{aligned}
$$




\section{Appendix $\mathrm{C}$}

\section{SLAG $d$-Cycles}

\section{C.1 The $d$-Cycle Equations}

A d-dimensional 'curve' $\Sigma^{(d)}$, embedded into $2 d$-dimensional flat space $\mathbb{R}^{2 d}$ with coordinates $x^{i}(i=1, \ldots, 2 d)$ can be described at least locally by the zero locus of $d$ real functions $f^{m}\left(x^{1}, \ldots, x^{2 d}\right)$ :

$$
\Sigma^{(d)}=\mathbb{V}\left(f^{1}, \ldots, f^{d}\right)=\left\{\left(x_{1}, \ldots, x_{2 d}\right) \mid f^{m}\left(x^{1}, \ldots, x^{2 d}\right)=0, m=1, \ldots, d\right\} .
$$

If one wants to deal with a so called supersymmetric $d$-cycle, the choice of the functions $f^{m}$ is highly constrained. To study these restrictions we first introduce $d$ real coordinates $\xi_{i}(i=1, \ldots, d)$ which parametrise the curve $\Sigma^{(d)}$. Furthermore we consider complex coordinates $u^{i}, u^{i}=x^{2 i-1}+i x^{2 i}$, of $\mathbb{C}^{d}$. Then the $d$-cycle can be characterised by making the complex $u^{i}$ to be functions of the real coordinates $\xi_{i}$, i.e. by the following embedding map $i$ from $\Sigma^{(d)}$ into $\mathbb{C}^{d}$ :

$$
i: \Sigma^{(d)} \longrightarrow \mathbb{C}^{d}: \quad \xi_{i} \longrightarrow u^{i}\left(\xi_{i}\right), \quad i=1, \ldots, d .
$$

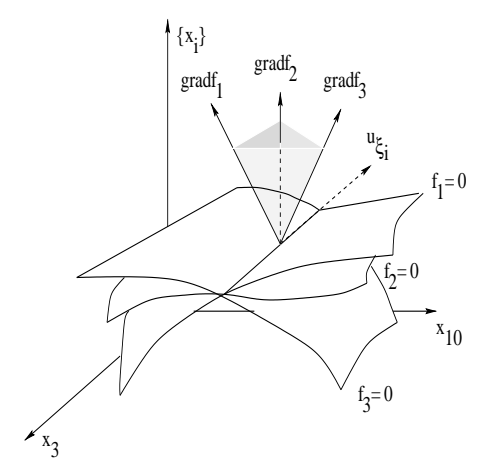

Fig. C.1: The intersecting configuration 
The intersection configuration (for the case $d=3$ ) is depicted in figure C.1.

Now by applying the partial derivative $\partial_{\xi_{k}}$ to the defining equations $f^{m}$ of the $d$-cycle, we get the following relations:

$$
\sum_{n=1}^{d}\left(f_{u^{n}}^{m} u_{\xi_{k}}^{n}+f_{\bar{u}^{n}}^{m} \bar{u}_{\xi_{k}}^{n}\right)=0
$$

(here $f_{u^{n}}^{m}=\frac{\partial f^{m}}{\partial u^{n}}, u_{\xi_{k}}^{n}=\frac{\partial u^{n}}{\partial \xi_{k}}$ ). These can be grouped into the following matrix expressions

$$
\left(\begin{array}{cccc}
f_{u^{1}}^{1} & f_{u^{2}}^{1} & \ldots & f_{u^{d}}^{1} \\
f_{u^{1}}^{2} & f_{u^{2}}^{2} & \ldots & f_{u^{d}}^{2} \\
\ldots & \ldots & \ldots & \ldots \\
f_{u^{1}}^{d} & f_{u^{2}}^{d} & \ldots & f_{u^{d}}^{d}
\end{array}\right) \cdot\left(\begin{array}{c}
u_{\xi_{k}}^{1} \\
u_{\xi_{k}}^{2} \\
\ldots \\
u_{\xi_{i}}^{d}
\end{array}\right)=(-1)^{d}\left(\begin{array}{cccc}
f_{\bar{u}^{1}}^{1} & f_{\bar{u}^{2}}^{1} & \ldots & f_{\bar{u}^{d}}^{1} \\
f_{\bar{u}^{1}}^{2} & f_{\bar{u}^{2}}^{2} & \ldots & f_{\bar{u}^{d}}^{2} \\
\ldots & \ldots & \ldots & \ldots \\
f_{\bar{u}^{1}}^{d} & f_{\bar{u}^{2}}^{d} & \ldots & f_{\bar{u}^{d}}^{d}
\end{array}\right) \cdot\left(\begin{array}{c}
\bar{u}_{\xi_{k}}^{1} \\
\bar{u}_{\xi_{k}}^{2} \\
\ldots \\
\bar{u}_{\xi_{k}}^{d}
\end{array}\right)
$$

We will denote the left matrix by $M$ and the right matrix as $\bar{M}$, henceforth. Note, the sign in front of $\bar{M}$ depends on the dimension $d$ of the cycle. With the help of these matrices we can express the bared derivatives by the unbared ones in the following way:

$$
\partial_{k} \bar{U}=(-1)^{d} \bar{M}^{-1} M \cdot \partial_{k} U=N \cdot \partial_{k} U .
$$

By definition $N$ shares the properties:

1. $N^{-1}=(-1)^{d} M^{-1} \bar{M}=\bar{N}$

2. $|\operatorname{det} N|=1$

Remembering the $d$-cycle should be supersymmetric we can ask for restrictions of the matrix $N$ following from this condition. It is well known that the notion of supersymmetric cycles [65] coincides with the notion of special Lagrangian submanifolds [68] which can be rephrased in terms of the embedding map $i: \Sigma^{(d)} \longrightarrow \mathbb{C}^{d}$ and the two conditions:

$$
\begin{array}{rlll}
i^{*} \Im \mathfrak{m} \Omega & = & 0 & \text { volume minimizing } \\
i^{*} \omega & = & 0 & \text { Lagrangian submanifold }
\end{array}
$$

With $\Omega=d u^{1} \wedge \ldots \wedge d u^{d}$ and $\omega=\frac{1}{2 i} \sum_{i} d u^{i} \wedge d \bar{u}^{i}$. The requirement of minimal volume reads

$$
\begin{aligned}
0=i^{*} \Im \mathfrak{m} \Omega & =\Im \mathfrak{m}\left(d u^{1}\left(\xi_{1}, \ldots, \xi_{d}\right) \wedge \ldots \wedge d u^{d}\left(\xi_{1}, \ldots, \xi_{d}\right)\right) \\
& =\Im \mathfrak{m}\left(\epsilon_{i_{1} \ldots i_{d}} u_{\xi_{1}}^{i_{1}} u_{\xi_{2}}^{i_{2}} \ldots u_{\xi_{d}}^{i_{d}}\right) d \xi^{1} \wedge \ldots \wedge d \xi^{d} \\
\Rightarrow \quad 0 & =\frac{1}{2 i}\left(\epsilon_{i_{1} \ldots i_{d}} u_{\xi_{1}}^{i_{1}} \ldots u_{\xi_{d}}^{i_{d}}-\epsilon_{i_{1} \ldots i_{d}} \bar{u}_{\xi_{1}}^{i_{1}} \ldots \bar{u}_{\xi_{d}}^{i_{d}}\right) \\
& =\frac{1}{2 i}\left(\epsilon_{i_{1} \ldots i_{d}} u_{\xi_{1}}^{i_{1}} \ldots u_{\xi_{d}}^{i_{d}}-\epsilon_{i_{1} \ldots i_{d}} N_{j_{1}}^{i_{1}} \ldots N_{j_{d}}^{i_{d}} u_{\xi_{1}}^{j_{1}} \ldots u_{\xi_{d}}^{j_{d}}\right) \\
& =\frac{1}{2 i}\left(\epsilon_{i_{1} \ldots i_{d}}-\epsilon_{j_{1} \ldots j_{d}} N_{i_{1}}^{j_{1}} \ldots N_{i_{d}}^{j_{d}}\right) u_{\xi_{1}}^{i_{1}} \ldots u_{\xi_{d}}^{i_{d}} \\
& =\frac{1}{2 i}(1-\operatorname{det} N) \cdot \frac{\partial\left(u^{1}, \ldots, u^{d}\right)}{\partial\left(\xi_{1}, \ldots, \xi_{d}\right)}
\end{aligned}
$$


which yields

$$
\left.\operatorname{det} N\right|_{\mathbb{V}\left(f^{1}, \ldots, f^{n}\right)}=1 \quad \text { or for short } \quad \operatorname{det} N \equiv 1 .
$$

For the calculation of the det-equation the following relation is useful.

$$
\operatorname{det} N \equiv 1 \Leftrightarrow \operatorname{det} M-(-1)^{d} \operatorname{det} \bar{M} \equiv 0
$$

Now we turn to the second equation. With the canonical Kähler (symplectic) form $\omega$, the pull back operation results in

$$
\begin{aligned}
0=i^{*} \omega & =\frac{1}{2 i} \sum_{i} d u^{i}\left(\xi_{1} \ldots \xi_{d}\right) \wedge d \bar{u}^{i}\left(\xi_{1} \ldots \xi_{d}\right) \\
& =\frac{1}{2 i} \sum_{i}\left(\sum_{k} u_{\xi_{k}}^{i} d \xi_{k}\right) \wedge\left(\sum_{l} \bar{u}_{\xi_{l}}^{i} d \xi_{l}\right) \\
& =\frac{1}{2 i} \sum_{k<l} \sum_{i}\left[u_{\xi_{k}}^{i} \bar{u}_{\xi_{l}}^{i}-u_{\xi_{l}}^{i} \bar{u}_{\xi_{k}}^{i}\right] d \xi_{k} \wedge d \xi_{l} \\
\Rightarrow \quad 0= & \sum_{i}\left[u_{\xi_{k}}^{i} \bar{u}_{\xi_{l}}^{i}-u_{\xi_{l}}^{i} \bar{u}_{\xi_{k}}^{i}\right] \\
= & \sum_{i}\left[u_{\xi_{k}}^{i}\left(\sum_{m} N_{m}^{i} u_{\xi_{l}}^{m}\right)-u_{\xi_{l}}^{i}\left(\sum_{m} N_{m}^{i} u_{\xi_{k}}^{m}\right)\right] \\
= & \sum_{i} u_{\xi_{k}}^{i}\left(\sum_{m} N_{m}^{i} u_{\xi_{l}}^{m}\right)-\sum_{i} u_{\xi_{l}}^{i}\left(\sum_{m} N_{m}^{i} u_{\xi_{k}}^{m}\right) \\
= & \sum_{i} u_{\xi_{k}}^{i}\left(\sum_{m} N_{m}^{i} u_{\xi_{l}}^{m}\right)-\sum_{m}\left(\sum_{i} u_{\xi_{l}}^{i} N_{m}^{i}\right) u_{\xi_{k}}^{m} \\
= & \sum_{i} u_{\xi_{k}}^{i}\left(\sum_{m} N_{m}^{i} u_{\xi_{l}}^{m}\right)-\sum_{i}\left(\sum_{m} u_{\xi_{l}}^{m} N^{T^{T}}{ }_{m}^{i}\right) u_{\xi_{k}}^{i} \\
= & \sum_{i, m}\left(N_{m}^{i}-N^{T}{ }_{m}^{i}\right) u_{\xi_{k}}^{i} u_{\xi_{l}}^{m}
\end{aligned}
$$

which is satisfied if we set $N \equiv N^{T}$. However, as it stands, this requirement is sufficient, only. Now we intend to give a proof that the condition is necessary, too.

To proof $N \equiv N^{T}$ we remember some facts from symplectic geometry especially various ways of characterising Lagrangian planes in symplectic vector spaces. The utility of this investigation rest on the simple observation that our conditions on the d-cycle to be a special Lagrangian submanifolds are in fact conditions on its tangent bundle, i.e. Lagrangian planes locally. 
To begin with, we consider a complex vector space $\mathbb{C}^{d}$ furnished with a Hermitian structure

$$
<x, y>=\sum_{i} x_{i} \bar{y}_{i}=g(x, y)+i \sigma(x, y)
$$

which splits into an Euclidean metric $g$ and a symplectic form $\sigma$. One can check that $\sigma$ coincides with

$$
\omega=\frac{1}{2 i} \sum_{i} d u^{i} \wedge d \bar{u}^{i} .
$$

given before. Therefore we identify both objects. The two-form $\omega$ is non degenerated, antisymmetric and bilinear. With help of $\omega$ we can define the notion of symplectic orthogonality.

Definition 1. The orthogonal complement of a vector subspace $E \in \mathbb{C}^{d}$ is defined by

$$
E^{\perp}=\left\{x \in \mathbb{C}^{d} \mid \omega(x, E)=0\right\}
$$

In the special case that $E=E^{\perp}$ we call $E$ a Lagrangian plane. Obviously on a Lagrangian plane the symplectic form restricts to zero. So we recognise the content of the constraint $i^{*} \omega=0$. It simply states that all tangent spaces to the supersymmetric cycle are Lagrangian planes embedded in the tangent space of the embedding space. Here we collect some facts:

1. $S p(E)$ operates transitively on Lagrangian planes

2. Since $U(d)$ preserves the Hermitian form, it is contained in $S p(E)$.

3. By $\mathcal{L}\left(\mathbb{C}^{d}\right)$ we denote the Graßmannian of Lagrangian planes

4. $\lambda \in \mathcal{L}\left(\mathbb{C}^{d}\right)$ is characterised by choosing an orthonormal basis $\left(a_{1}, \ldots, a_{n}\right)$ with respect to the Euclidean metric $g$. But then it is orthonormal with respect to the Hermitian form, too:

$$
<a_{i}, a_{j}>=g\left(a_{i}, a_{j}\right)+i \omega\left(a_{i}, a_{j}\right) \stackrel{!}{=} \delta_{i j},
$$

i.e. the matrix $a=\left(a_{1}, \ldots, a_{n}\right)$ is unitary. The other direction works, too. Hence

$$
\lambda \in \mathcal{L}\left(\mathbb{C}^{d}\right) \Leftrightarrow \exists a \in U(d), \lambda=a\left(\mathbb{R}^{d}\right)
$$




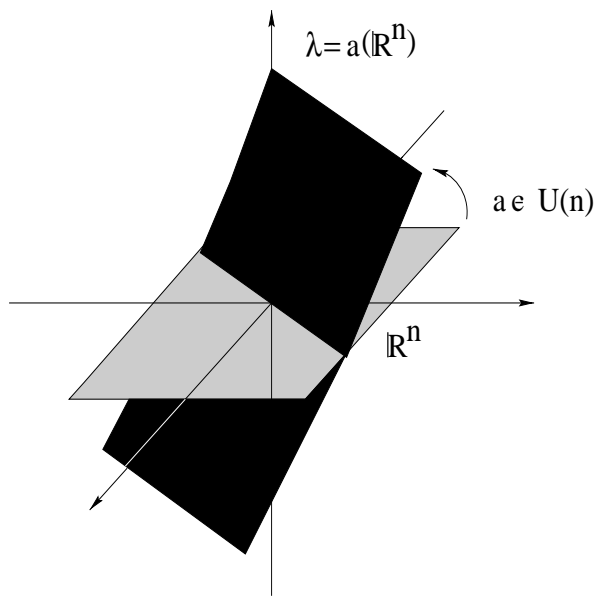

Fig. C.2: The operation of $a$ on Lagrangian planes

5. Obviously each Lagrangian plane will be stabilised by any element in $O(n)$, i.e. we can regard the Graßmannian of Lagrangian planes as the quotient space

$$
\mathcal{L}\left(\mathbb{C}^{d}\right)=\frac{U(d)}{O(d)}
$$

How can we define a projection from $U(d)$ onto $\mathcal{L}\left(\mathbb{C}^{d}\right)$ ? We observe that two elements $a$ and $a^{\prime}$ determine the same Lagrangian plane, iff

$$
\lambda=a\left(\mathbb{R}^{d}\right)=a^{\prime}\left(\mathbb{R}^{d}\right) \Leftrightarrow a \bar{a}^{-1}=a^{\prime} \bar{a}^{\prime-1},
$$

which is constant on the $O(d)$-orbits of the fibration. Now we can identify $\mathcal{L}\left(\mathbb{C}^{d}\right)$ with the image of the projection map

$$
\begin{aligned}
\pi: U(d) & \rightarrow \mathcal{L}\left(\mathbb{C}^{d}\right) \\
a & \mapsto \lambda=a \bar{a}^{-1}
\end{aligned}
$$

By abuse of language we denote the matrix representative $a \bar{a}^{-1}$ of the Lagrangian plane $\lambda=a\left(\mathbb{R}^{n}\right)$ by $\lambda$ again. But how can we associate the geometrical object with this artificial matrix representative? The connection between the matrix $\lambda$ on the one side and the concrete Lagrangian plane $\lambda$ on the other side is given through the central equation

$$
x \in \lambda \Leftrightarrow x=\lambda \bar{x}
$$

In the last formula we recognise the familiar equation (C.3). But now we know, that we can represent $\lambda$ as $\lambda=a \bar{a}^{-1}$ and this yields straight forward

$$
\begin{aligned}
\lambda^{+} & =\bar{a}^{-1^{+}} a^{+}=a^{-1^{T}} a^{-1}=\bar{a} a^{-1}=\bar{\lambda} \\
\Rightarrow \lambda^{T} & =\lambda
\end{aligned}
$$


But then we can finally conclude by identifying $\lambda=N^{-1}$ and performing some mild manipulations that

$$
N \equiv N^{T}
$$

In summary, all what we have done so far can be formulated in a short but important proposition which is the starting point for all further computations:

Proposition: $A$ d-cycle, represented as an intersection of $d$ real valued functions is supersymmetric, iff $N \equiv N^{T}$ and $\operatorname{det} N \equiv 1$.

It will turn out to be very useful to reformulate the last proposition $N \equiv N^{T}$ in a different, but equivalent way. Namely, it is not difficult to show that the requirement $N \equiv N^{T}$ is equivalent to the condition that the matrix $M M^{+}$should be real modulo $I(\mathbb{V})$. To prepare this reformulation we remark that by the split of the coordinates of $\mathbb{R}^{2 d}$ into the coordinates of $\mathbb{C}^{d}$ they inherit an intrinsic meaning as the spatial and momentum variables of symplectic geometry. This is given by

$$
u^{i}=q^{i}+i p^{i}
$$

i.e. the real part of $u^{i}$ gets the meaning of a spatial coordinate whereas the $p^{i}$ is a momentum variable. Then we are free to define the convenient Poisson brackets of phase-space functions $\{f, g\}$. This is done in the standard way as

$$
\{f, g\}=\sum_{i=1}^{d}\left(\frac{\partial f}{\partial q^{i}} \frac{\partial g}{\partial p^{i}}-\frac{\partial f}{\partial p^{i}} \frac{\partial g}{\partial q^{i}}\right)=\sum_{i=1}^{d}\left(f_{2 i-1} g_{2 i}-f_{2 i} g_{2 i-1}\right)
$$

where $f_{2 i-1}=\frac{\partial f}{\partial q^{i}}=\frac{\partial f}{\partial x^{2 i-1}}$ and $f_{2 i}=\frac{\partial f}{\partial p^{i}}=\frac{\partial f}{\partial x^{2 i}}$. Then the matrix $M M^{+}$reads

$$
\left(M M^{+}\right)_{m n}=<\nabla f^{m}, \nabla f^{n}> \pm i \cdot\left\{f^{m}, f^{n}\right\} .
$$

So $M M^{+}$is a real matrix modulo $I(\mathbb{V})$, i.e. $N \equiv N^{T}$, if all Poisson brackets among the defining functions $f^{m}$ and $f^{n}$ vanish:

$$
\left\{f^{m}, f^{n}\right\} \equiv 0 .
$$

So we get a more suitable set of equations for concrete calculations.

Corollary: A d-cycle, represented as an intersection of $d$ real valued functions is supersymmetric, iff $\left\{f^{i}, f^{j}\right\} \equiv 0$ and $\operatorname{det} N \equiv 1$ 


\section{C.2 An Algebraic Identity}

$$
\begin{aligned}
\operatorname{det} g= & {\left[1+\left(\frac{\partial X_{1}}{\partial q_{1}}\right)^{2}+\left(\frac{\partial X_{2}}{\partial q_{1}}\right)^{2}\right]\left[1+\left(\frac{\partial X_{1}}{\partial q_{2}}\right)^{2}+\left(\frac{\partial X_{2}}{\partial q_{2}}\right)^{2}\right]-\left[\frac{\partial X_{1}}{\partial q_{1}} \frac{\partial X_{1}}{\partial q_{2}}+\frac{\partial X_{2}}{\partial q_{1}} \frac{\partial X_{2}}{\partial q_{2}}\right]^{2} } \\
= & 1+\left(\frac{\partial X_{1}}{\partial q_{1}}\right)^{2}+\left(\frac{\partial X_{2}}{\partial q_{1}}\right)^{2}+\left(\frac{\partial X_{1}}{\partial q_{2}}\right)^{2}+\left(\frac{\partial X_{2}}{\partial q_{2}}\right)^{2} \\
& +\left[\left(\frac{\partial X_{1}}{\partial q_{1}}\right)^{2}+\left(\frac{\partial X_{2}}{\partial q_{1}}\right)^{2}\right]\left[\left(\frac{\partial X_{1}}{\partial q_{2}}\right)^{2}+\left(\frac{\partial X_{2}}{\partial q_{2}}\right)^{2}\right]-\left[\frac{\partial X_{1}}{\partial q_{1}} \frac{\partial X_{1}}{\partial q_{2}}+\frac{\partial X_{2}}{\partial q_{1}} \frac{\partial X_{2}}{\partial q_{2}}\right]^{2} \\
= & 1+\left(\frac{\partial X_{1}}{\partial q_{1}}\right)^{2}+\left(\frac{\partial X_{2}}{\partial q_{1}}\right)^{2}+\left(\frac{\partial X_{1}}{\partial q_{2}}\right)^{2}+\left(\frac{\partial X_{2}}{\partial q_{2}}\right)^{2}+\left(\frac{\partial X_{1}}{\partial q_{1}} \frac{\partial X_{2}}{\partial q_{2}}-\frac{\partial X_{2}}{\partial q_{1}} \frac{\partial X_{1}}{\partial q_{2}}\right)^{2} \\
= & \left(\frac{\partial X_{1}}{\partial q_{1}}+\frac{\partial X_{2}}{\partial q_{2}}\right)^{2}-2 \frac{\partial X_{1}}{\partial q_{1}} \frac{\partial X_{2}}{\partial q_{2}}+\left(\frac{\partial X_{1}}{\partial q_{2}}-\frac{\partial X_{2}}{\partial q_{1}}\right)^{2}+2 \frac{\partial X_{1}}{\partial q_{2}} \frac{\partial X_{2}}{\partial q_{1}}+\left(\frac{\partial X_{1}}{\partial q_{1}} \frac{\partial X_{2}}{\partial q_{2}}-\frac{\partial X_{2}}{\partial q_{1}} \frac{\partial X_{1}}{\partial q_{2}}\right)^{2}+1 \\
= & \left(\frac{\partial X_{1}}{\partial q_{1}}+\frac{\partial X_{2}}{\partial q_{2}}\right)^{2}+\left(\frac{\partial X_{1}}{\partial q_{2}}-\frac{\partial X_{2}}{\partial q_{1}}\right)^{2}+\left(1-\frac{\partial X_{1}}{\partial q_{1}} \frac{\partial X_{2}}{\partial q_{2}}+\frac{\partial X_{2}}{\partial q_{1}} \frac{\partial X_{1}}{\partial q_{2}}\right)^{2} \\
= & {\left[f^{*} \Im \mathfrak{m} \Omega\right]^{2}+\left[f^{*} \Re \mathfrak{e} \Omega\right]^{2}+\left[f^{*} \omega\right]^{2} }
\end{aligned}
$$

\section{C.3 Graßmannians of 2-Planes in $\mathbb{R}^{4}$}

The Graßmannian of 2-planes in $\mathbb{R}^{4}$ is defined through:

$$
G(2,4)=\left\{\xi \wedge \eta \mid \xi, \eta \in \Omega^{1}\left(\mathbb{R}^{4}\right)\right\} \subset \Lambda^{2} \mathbb{R}^{4}
$$

Each element is a plane generated by the vectors corresponding to the 1-forms $\xi$ or $\eta$, respectively. To investigate the structure of the manifold $G(2,4)$ we take notice of the action of the Hodge star operator $(*)$ on $\Lambda^{2} \mathbb{R}^{4}$. Since $*^{2}=\mathbb{1}$ there is a split of $\Lambda^{2} \mathbb{R}^{4}$ into eigenspaces of $*$.

$$
\begin{array}{clc}
\multicolumn{3}{c}{\Lambda^{2} \mathbb{R}^{4}} \\
P_{+} \swarrow & \searrow P_{-} \\
\Lambda_{+}^{2} \mathbb{R}^{4} & \Lambda_{-}^{2} \mathbb{R}^{4}
\end{array}
$$

Each element $x$ of $G(2,4)$ is of the form $x=\xi \wedge \eta$, where the vectors $\xi$ and $\eta$ can be chosen to be orthonormal, i.e.

$$
x \in G(2,4), \text { iff } x \wedge x=0 .
$$

Using the decomposition into eigenspaces each $x \in G(2,4)$ can be written as

$$
x=P_{+} x+P_{-} x
$$


and it is easy to see that this splitting defines a bijection of $G(2,4)$ onto $S_{+}^{2} \times S_{-}^{2}$.

To each point of $x_{J} \in S_{+}^{2}$ one can associate a complex structure.

$$
<J \xi, \eta>=<x_{J}, \xi \wedge \eta>
$$

and this is a bijection, too. It is is simple exercise to compute $J$ in terms of $x_{J}$ which is given by

$$
J=\left(\begin{array}{cccc}
0 & -a_{1} & -a_{2} & -a_{3} \\
a_{1} & 0 & -a_{3} & a_{2} \\
a_{2} & a_{3} & 0 & -a_{1} \\
a_{3} & -a_{2} & a_{1} & 0
\end{array}\right)
$$

for $x_{J}=\left(a_{1}, a_{2}, a_{3}\right)$ the coordinates of $x_{J}$. Then a real vector can be maid into a complex one by the map

$$
\left(b_{1}, b_{2}, b_{3}, b_{4}\right)^{T} \mapsto\left(\begin{array}{c}
b_{1}+i \cdot b_{2} \\
b_{3}+i \cdot b_{4}
\end{array}\right) .
$$

After the choice of a complex structure, one is able to talk about the Graßmannian of Lagrangian planes $\mathcal{L}\left(\mathbb{R}^{4}\right)$, defined by those $\xi \wedge \eta$ satisfying

$$
\omega_{J}(\xi, \eta)=<J \xi, \eta>=<x_{J}, \xi \wedge \eta>=0 .
$$

After some short calculations it becomes clear, that

$$
\mathcal{L}\left(\mathbb{R}^{4}\right)=S_{+}^{1} \times S_{-}^{2}
$$

with $S_{+}^{1}=S_{+}^{2} \cap x_{J}^{\perp}$, i.e. $S_{+}^{1}$ is the intersection of $S_{+}^{2}$ with the orthogonal complement of $x_{J} \in S_{+}^{2}$.

Taking into account that $\mathcal{L}\left(\mathbb{R}^{4}\right)=U(2) / S O(2)$ as a homogeneous space one can reinterpret the topology of $\mathcal{L}\left(\mathbb{R}^{4}\right)$ in terms of $U(2)$ as indicated below:

$$
\begin{array}{ccc} 
& & \\
& & \\
& & \\
& & \\
& & \\
\text { Phase } \swarrow(1) \times S U(2) & \\
S_{+}^{1} & \searrow \text { Hopf } \\
& & S_{-}^{2}
\end{array}
$$

Each element of $U(2)$ can be represented by a $2 \times 2$-matrix:

$$
U=\left(\begin{array}{cc}
a_{1}+i \cdot a_{2} & b_{1}+i \cdot b_{2} \\
a_{3}+i \cdot a_{4} & b_{3}+i \cdot b_{4}
\end{array}\right)
$$


The projection of the corresponding plane $a \wedge b$ into the "+"-eigenspace can be parametrised by the angle $\alpha$ of $S_{+}^{1}$ as follows:

$$
\begin{aligned}
P_{+}(a \wedge b)= & \underbrace{\left\{a_{[1} b_{2]}+a_{[3} b_{4]}\right\}}_{0} P_{+}\left(d x^{1} \wedge d x^{2}\right) \\
& +\underbrace{\left\{a_{[1} b_{3]}-a_{[2} b_{4]}\right\}}_{\cos \alpha} P_{+}\left(d x^{1} \wedge d x^{3}\right) \\
& +\underbrace{\left\{a_{[2} b_{3]}+a_{[1} b_{4]}\right\}}_{\sin \alpha} P_{+}\left(d x^{2} \wedge d x^{3}\right)
\end{aligned}
$$

On the other side one can compute the determinant of $U(2)$ which yields

$$
\operatorname{det} U=\underbrace{\left\{a_{[1} b_{3]}-a_{[2} b_{4]}\right\}}_{\cos \alpha}+i \underbrace{\left\{a_{[1} b_{4]}+a_{[2} b_{3]}\right\}}_{\sin \alpha}=e^{i \alpha}
$$

A $S U(2)-S L A G$ calibrated surface would be one with a constant phase function. 


\section{Bibliography}

[1] E. Cremmer, B. Julia, and J. Scherk, "Supergravity theory in 11 dimensions," Phys. Lett. B76 (1978) 409-412.

[2] E. Witten, "Solutions of four-dimensional field theories via M-theory," Nucl. Phys. B500 (1997) 3, hep-th/9703166.

[3] M. R. Douglas and G. Moore, "D-Branes, Quivers, and ALE Instantons," hep-th/9603167.

[4] M. Aganagic, A. Karch, D. Lüst, and A. Miemiec, "Mirror symmetries for brane configurations and branes at singularities," hep-th/9903093.

[5] A. Hanany and A. Zaffaroni, "On the realization of chiral four-dimensional gauge theories using branes," JHEP 05 (1998) 001, hep-th/9801134.

[6] D. Lüst and A. Miemiec, "Supersymmetric M5-branes with H field," hep-th/9912065.

[7] P. S. Howe, E. Sezgin, and P. C. West, "Covariant field equations of the M-theory five-brane," Phys. Lett. B399 (1997) 49, hep-th/9702008.

[8] O. Bärwald, N. D. Lambert, and P. C. West, "A calibration bound for the M-theory fivebrane," Phys. Lett. B463 (1999) 33, hep-th/9907170.

[9] A. Karch, D. Lüst, and A. Miemiec, "N = 1 supersymmetric gauge theories and supersymmetric 3-cycles," Nucl. Phys. B553 (1999) 483, hep-th/9810254.

[10] A. Hanany and A. M. Uranga, "Brane boxes and branes on singularities," JHEP 05 (1998) 013, hep-th/9805139.

[11] E. G. Gimon and M. Gremm, "A note on brane boxes at finite string coupling," Phys. Lett. B433 (1998) 318, hep-th/9803033.

[12] J. Maldacena, "The large N limit of superconformal field theories and supergravity," Adv. Theor. Math. Phys. 2 (1998) 231-252, hep-th/9711200.

[13] S. Kachru and E. Silverstein, "4d conformal theories and strings on orbifolds," Phys. Rev. Lett. 80 (1998) 4855, hep-th/9802183.

[14] A. Lawrence, N. Nekrasov, and C. Vafa, "On conformal field theories in four dimensions," Nucl. Phys. B533 (1998) 199, hep-th/9803015. 
[15] I. R. Klebanov and E. Witten, "Superconformal field theory on threebranes at a Calabi-Yau singularity," Nucl. Phys. B536 (1998) 199, hep-th/9807080.

[16] A. M. Uranga, "Brane configurations for branes at conifolds," JHEP 01 (1999) 022, hep-th/9811004.

[17] A. Karch, D. Lüst, and A. Miemiec, "New N=1 superconformal field theories and their supergravity description," Phys. Lett. B454 (1999) 265, hep-th/9901041.

[18] A. Khavaev, K. Pilch, and N. P. Warner, "New vacua of gauged N $=8$ supergravity in five dimensions," hep-th/9812035.

[19] P. K. Townsend, "M-theory from its superalgebra," Cargese lectures (1997) hep-th/9712004.

[20] J. D. Lykken, "Introduction to supersymmetry," Lectures given at TASI-96 (1996) hep-th/9612114.

[21] M. J. Duff and K. S. Stelle, "Multi-membrane solutions of D = 11 supergravity," Phys. Lett. B253 (1991) 113-118.

[22] R. Güven, "Black p-brane solutions of D = 11 supergravity theory," Phys. Lett. B276 (1992) 49.

[23] R. Arnowitt, S. Deser, and C. Misner, Gravitation: An Introduction to the currecnt research. ed. by L.Witten, New York (Wiley), 1962.

[24] M. Aganagic, J. Park, C. Popescu, and J. H. Schwarz, "World-volume action of the M-theory five-brane," Nucl. Phys. B496 (1997) 191, hep-th/9701166.

[25] D. Sorokin, "Superbranes and superembeddings," Review (1999) hep-th/9906142.

[26] J. P. Gauntlett, N. D. Lambert, and P. C. West, "Supersymmetric five-brane solitons," Adv. Theor. Math. Phys. 3 (1999) 91, hep-th/9811024.

[27] M. Huq and M. A. Namazie, "Kaluza-Klein supergravity in ten dimensions," Class. Quant. Grav. 2 (1985) 293.

[28] J. H. Schwarz and P. C. West, "Symmetries and transformations of chiral N=2 D = 10 supergravity," Phys. Lett. B126 (1983) 301.

[29] T. H. Buscher, "A symmetry of the string background field equations," Phys. Lett. B194 (1987) 59.

[30] T. H. Buscher, "Path integral derivation of quantum duality in nonlinear sigma models," Phys. Lett. B201 (1988) 466.

[31] K. Kikkawa and M. Yamasaki, "Casimir effects in superstring theories," Phys. Lett. B149 (1984) 357.

[32] A. Font, L. E. Ibáñez, D. Lüst, and F. Quevedo, "Strong - weak coupling duality and nonperturbative effects in string theory," Phys. Lett. B249 (1990) 35-43. 
[33] J. H. Schwarz, "An SL(2,Z) multiplet of type IIB superstrings," Phys. Lett. B360 (1995) $13-18$, hep-th/9508143.

[34] H. Ooguri and C. Vafa, "Two-Dimensional Black Hole and Singularities of CY Manifolds," Nucl. Phys. B463 (1996) 55-72, hep-th/9511164.

[35] M. Bershadsky, C. Vafa, and V. Sadov, "D-Strings on D-Manifolds," Nucl. Phys. B463 (1996) 398-414, hep-th/9510225.

[36] A. Hanany and E. Witten, "Type IIB superstrings, BPS monopoles, and three-dimensional gauge dynamics," Nucl. Phys. B492 (1997) 152-190, hep-th/9611230.

[37] B. Andreas, G. Curio, and D. Lüst, "The Neveu-Schwarz five-brane and its dual geometries," JHEP 10 (1998) 022, hep-th/9807008.

[38] K. Dasgupta and S. Mukhi, "Brane constructions, conifolds and M-theory," Nucl. Phys. B551 (1999) 204, hep-th/9811139.

[39] A. Karch, D. Lüst, and D. Smith, "Equivalence of geometric engineering and Hanany-Witten via fractional branes," Nucl. Phys. B533 (1998) 348, hep-th/9803232.

[40] E. Lopez, "A family of $\mathrm{N}=1 \mathrm{SU}(\mathrm{N}) * * \mathrm{k}$ theories from branes at singularities," JHEP 02 (1999) 019, hep-th/9812025.

[41] J. Polchinski, "Dirichlet-Branes and Ramond-Ramond Charges," Phys. Rev. Lett. 75 (1995) 4724-4727, hep-th/9510017.

[42] R. G. Leigh, "Dirac-Born-Infeld action from Dirichlet sigma model," Mod. Phys. Lett. A4 (1989) 2767.

[43] E. S. Fradkin and A. A. Tseytlin, "Nonlinear Electrodynamics From Quantized Strings," Phys. Lett. B163 (1985) 123.

[44] E. Witten, "Bound states of strings and p-branes," Nucl. Phys. B460 (1996) 335-350, hep-th/9510135.

[45] G. T. Horowitz and A. Strominger, "Black Strings and p-Branes," Nucl. Phys. B360 (1991) 197-209.

[46] M. R. Douglas, B. R. Greene, and D. R. Morrison, "Orbifold Resolution by D-Branes," Nucl. Phys. B506 (1997) 84, hep-th/9704151.

[47] D. R. Morrison and M. R. Plesser, "Non-spherical horizons. I," Adv. Theor. Math. Phys. 3 (1999) 1, hep-th/9810201.

[48] A. Strominger, S. Yau, and E. Zaslow, "Mirror symmetry is T-duality," Nucl. Phys. B479 (1996) 243-259, hep-th/9606040.

[49] N. C. Leung and C. Vafa, "Branes and toric geometry," Adv. Theor. Math. Phys. 2 (1998) 91, hep-th/9711013. 
[50] S. Katz, P. Mayr, and C. Vafa, "Mirror symmetry and exact solution of $4 \mathrm{D} \mathrm{N}=2$ gauge theories. I," Adv. Theor. Math. Phys. 1 (1998) 53-114, hep-th/9706110.

[51] W. Fulton, Introduction to toric varieties. Princeton University Press, 1993.

[52] E. Witten, "Phases of N $=2$ theories in two dimensions," Nucl. Phys. B403 (1993) 159-222, hep-th/9301042.

[53] P. S. Aspinwall and B. R. Greene, "On the geometric interpretation of $\mathrm{N}=2$ superconformal theories," Nucl. Phys. B437 (1995) 205-230, hep-th/9409110.

[54] D. A. Cox, "Recent developments in toric geometry," Proc. Symp. Pure Math. 62(pt.2) (1997) 389-436, alg-geom/9606016.

[55] D. A. Cox, "The Homogeneous Coordinate Ring of a Toric Variety," J. Algebr. Geom. 4 No.1 (1995) 17-50, alg-geom/9210008.

[56] S. Katz, A. Klemm, and C. Vafa, "Geometric engineering of quantum field theories," Nucl. Phys. B497 (1997) 173-195, hep-th/9609239.

[57] T. M. Chiang, A. Klemm, S. T. Yau, and E. Zaslow, "Local mirror symmetry: Calculations and interpretations," hep-th/9903053.

[58] S. Elitzur, A. Giveon, and D. Kutasov, "Branes and N = 1 duality in string theory," Phys. Lett. B400 (1997) 269-274, hep-th/9702014.

[59] W. Lerche, "Introduction to Seiberg-Witten theory and its stringy origin," Nucl. Phys. Proc. Suppl. 55B (1997) 83-117, hep-th/9611190.

[60] A. Sen, "A non-perturbative description of the Gimon-Polchinski orientifold," Nucl. Phys. B489 (1997) 139-159, hep-th/9611186.

[61] K. Hori, H. Ooguri, and C. Vafa, "Non-Abelian conifold transitions and $\mathrm{N}=4$ dualities in three dimensions," Nucl. Phys. B504 (1997) 147, hep-th/9705220.

[62] R. de Mello Koch, K. Oh, and R. Tatar, "Moduli space for conifolds as intersection of orthogonal D6 branes," Nucl. Phys. B555 (1999) 457, hep-th/9812097.

[63] H. Ooguri and C. Vafa, "Geometry of $\mathrm{N}=1$ dualities in four dimensions," Nucl. Phys. B500 (1997) 62, hep-th/9702180.

[64] R. von Unge, "Branes at generalized conifolds and toric geometry," JHEP 02 (1999) 023, hep-th/9901091.

[65] K. Becker, M. Becker, and A. Strominger, "Five-branes, membranes and nonperturbative string theory," Nucl. Phys. B456 (1995) 130-152, hep-th/9507158.

[66] G. W. Gibbons and G. Papadopoulos, "Calibrations and intersecting branes," Commun. Math. Phys. 202 (1999) 593, hep-th/9803163.

[67] J. P. Gauntlett, N. D. Lambert, and P. C. West, "Branes and calibrated geometries," Commun. Math. Phys. 202 (1999) 571, hep-th/9803216. 
[68] R. Harvey and H. B. Lawson, "Calibrated geometries," Acta Mathematica 148 (1982) $47-157$.

[69] J. M. Figueroa-O'Farrill, "Intersecting brane geometries," Review (1998) hep-th/9806040.

[70] M. Marino, R. Minasian, G. Moore, and A. Strominger, "Nonlinear instantons from supersymmetric p-branes," hep-th/9911206.

[71] V. Guillemin and S. Sternberg, "Geometric Asymptotics," Mathemaical Surveys and Monographs Nr. 14. (1977).

[72] O. Bärwald, N. D. Lambert, and P. C. West, "On the energy momentum tensor of the M-theory fivebrane," Phys. Lett. B459 (1999) 125, hep-th/9904097.

[73] J. Gutowski, G. Papadopoulos, and P. K. Townsend, "Supersymmetry and generalized calibrations," Phys. Rev. D60 (1999) 106006, hep-th/9905156.

[74] M. Cederwall, U. Gran, M. Holm, and B. E. W. Nilsson, "Finite tensor deformations of supergravity solitons," JHEP 02 (1999) 003, hep-th/9812144.

[75] N. Seiberg and E. Witten, "Electric - magnetic duality, monopole condensation, and confinement in N=2 supersymmetric Yang-Mills theory," Nucl. Phys. B426 (1994) 19-52, hep-th/9407087.

[76] J. de Boer, K. Hori, H. Ooguri, and Y. Oz, "Kähler potential and higher derivative terms from M-theory five-brane," Nucl. Phys. B518 (1998) 173, hep-th/9711143.

[77] K. Hori, H. Ooguri, and Y. Oz, "Strong coupling dynamics of four-dimensional N = 1 gauge theories from M-theory fivebrane," Adv. Theor. Math. Phys. 1 (1998) 1-52, hep-th/9706082.

[78] E. Witten, "Branes and the dynamics of QCD," Nucl. Phys. B507 (1997) 658, hep-th/9706109.

[79] A. Brandhuber, N. Itzhaki, V. Kaplunovsky, J. Sonnenschein, and S. Yankielowicz, "Comments on the M-theory approach to N $=1$ SQCD and brane dynamics," Phys. Lett. B410 (1997) 27, hep-th/9706127.

[80] K. Landsteiner, E. Lopez, and D. A. Lowe, "Duality of chiral N = 1 supersymmetric gauge theories via branes," JHEP 02 (1998) 007, hep-th/9801002.

[81] I. Brunner, A. Hanany, A. Karch, and D. Lüst, "Brane dynamics and chiral non-chiral transitions," Nucl. Phys. B528 (1998) 197, hep-th/9801017.

[82] B. Kol, "5d field theories and M-theory," JHEP 11 (1999) 026, hep-th/9705031.

[83] R. G. Leigh and M. J. Strassler, "Exactly marginal operators and duality in four-dimensional N=1 supersymmetric gauge theory," Nucl. Phys. B447 (1995) 95-136, hep-th/9503121.

[84] A. Hanany, M. J. Strassler, and A. M. Uranga, "Finite theories and marginal operators on the brane," JHEP 06 (1998) 011, hep-th/9803086. 
[85] O. Aharony and A. Hanany, "Branes, superpotentials and superconformal fixed points," Nucl. Phys. B504 (1997) 239, hep-th/9704170.

[86] O. Aharony, A. Hanany, K. Intriligator, N. Seiberg, and M. J. Strassler, "Aspects of N=2 supersymmetric gauge theories in three dimensions," Nucl. Phys. B499 (1997) 67, hep-th/9703110.

[87] H. García-Compeán and A. M. Uranga, "Brane box realization of chiral gauge theories in two dimensions," Nucl. Phys. B539 (1999) 329, hep-th/9806177.

[88] A. Armoni and A. Brandhuber, "Comments on (non-)chiral gauge theories and type IIB branes," Phys. Lett. B438 (1998) 261, hep-th/9803186.

[89] R. C. McLean, "Deformation of calibrated Submanifolds," Comm. Anal. Geom. 6 (1998) $705-747$.

[90] K. Intriligator and N. Seiberg, "Lectures on supersymmetric gauge theories and electricmagnetic duality," Nucl. Phys. Proc. Suppl. 45BC (1996) 1-28, hep-th/9509066.

[91] S. S. Gubser, I. R. Klebanov, and A. M. Polyakov, "Gauge theory correlators from non-critical string theory," Phys. Lett. B428 (1998) 105, hep-th/9802109.

[92] E. Witten, "Anti-de Sitter space and holography," Adv. Theor. Math. Phys. 2 (1998) 253-291, hep-th/9802150.

[93] M. Henningson and K. Skenderis, "The holographic Weyl anomaly," JHEP 07 (1998) 023, hep-th/9806087.

[94] S. S. Gubser, "Einstein manifolds and conformal field theories," Phys. Rev. D59 (1999) 025006, hep-th/9807164.

[95] L. Girardello, M. Petrini, M. Porrati, and A. Zaffaroni, "Novel local CFT and exact results on perturbations of $\mathrm{N}=4$ Super Yang-Mills from AdS dynamics," JHEP 12 (1998) 022, hep-th/9810126.

[96] J. Distler and F. Zamora, "Non-supersymmetric conformal field theories from stable Anti-de Sitter spaces," Adv. Theor. Math. Phys. 2 (1999) 1405, hep-th/9810206.

[97] M. Günaydin, L. J. Romans, and N. P. Warner, "Gauged N=8 supergravity in five-dimensions," Phys. Lett. B154 (1985) 268.

[98] D. Z. Freedman, S. S. Gubser, K. Pilch, and N. P. Warner, "Renormalization group flows from holography supersymmetry and a c-theorem," hep-th/9904017.

[99] R. Donagi and E. Witten, "Supersymmetric Yang-Mills Theory and Integrable Systems," Nucl. Phys. B460 (1996) 299-334, hep-th/9510101.

[100] M. T. Grisaru, W. Siegel, and M. Roček, "Improved methods for supergraphs," Nucl. Phys. B159 (1979) 429.

[101] N. Seiberg, "Naturalness versus supersymmetric nonrenormalization theorems," Phys. Lett. B318 (1993) 469-475, hep-ph/9309335. 
[102] M. A. Shifman and A. I. Vainshtein, "Solution of the anomaly puzzle in susy gauge theories and the Wilson operator expansion," Nucl. Phys. B277 (1986) 456.

[103] M. A. Shifman and A. I. Vainshtein, "On holomorphic dependence and infrared effects in supersymmetric gauge theories," Nucl. Phys. B359 (1991) 571-580.

[104] D. Anselmi, D. Z. Freedman, M. T. Grisaru, and A. A. Johansen, "Nonperturbative formulas for central functions of supersymmetric gauge theories," Nucl. Phys. B526 (1998) 543, hep-th/9708042. 


\section{Zusammenfassung}

Der Gegenstand dieser Arbeit ist die Untersuchung von supersymmetrischen Eichtheorien durch die Anwendung geometrischer Methoden, die sich aus der Einbettung der Eichtheorien in die Stringtheorie ergeben. Das entscheidende Bindeglied ist hierbei die Niederenergiedynamik sogenannter Branes. Branes sind solitonische Lösungen der Bewegungsgleichungen in effektiven Stringtheorien (Supergravitation). Wir betrachten Branelösungen der IIA und IIB Supergravitation sowie der 11 dimensionalen Supergravition (M-Theorie). Die M-Theorie wird aus zwei Gründen eingeführt. Erstens gestattet sie es, eine vereinheitlichte Sicht auf die recht verwickelten Verhältnisse zu gewinnen. So lassen sich beispielsweise durch Kombination von Kaluza-Klein Mechanismus und T-Dualität alle IIA und IIB-Branes aus nur zwei elementaren M-Brane Lösungen, der M2 und M5-Brane, herleiten. Zweitens enthalten die klassischen Lösungen der M5-Brane die Informationen über die Quanteneffekte der Eichtheorien auf den IIA-Branes. Die folgenden Kapitel diskutieren verschiedene Aspekte dieses Programms.

Kapitel 1 faßt den theoretischen Hintergrund knapp zusammen. Es werden alle notwendigen Objekte zur Einbettung von Eichtheorien in die Stringtheorie eingeführt. Einen zentralen Platz nimmt dabei die Diskussion der Weltvolumentheorie der M5-Brane ein, die für die Kapitel 3 und 4 unentbehrlich wird. $S$ und $T$-Dualität werden benötigt, um die Beziehung zwischen Systemen von Neveu-Schwarz-Branes (NS-Branes) und singulären Raumzeiten zu beleuchten. Neben den NS-Branes treten noch sogenannte DirichletBranes (D-Branes) auf. Diese sind wesentlich leichter als NS-Branes und können daher als Testladungen in der singulären Raumzeit angesehen werden. Die Weltvolumentheorie von D-Branes ist eine Eichtheorie. Für den Fall, daß die Raumzeit die Struktur einer Orbifold $\mathcal{O}_{k l}=\mathbb{C}^{3} / \mathbb{Z}_{k} \times \mathbb{Z}_{l}$ hat, wird die Eichtheorie von $n$ parallelen D3-Branes explizit berechnet.

In Kapitel 2 untersuchen wir die Eichtheorie von D3-Branes an allgemeineren Singularitäten. Für zwei Arten von Singularitäten werden die T-dualen NS5-Branekonfigurationen untersucht: Quotienten aus der Conifoldsingularität $\mathcal{C}$ und der Gruppe $\Gamma=\mathbb{C}^{3} / \mathbb{Z}_{k} \times \mathbb{Z}_{l}$, d.h. $\mathcal{C}_{k l}=\mathcal{C} / \Gamma$, und verallgemeinerte Conifoldsingularitäten $\mathcal{G}_{k l}$. Es erweist sich, daß der Blowup (bzw. die Deformation) von $\mathcal{C}_{k l} T$-dual zu einer BraneBox (bzw. einem Intervall) aus NS5/NS5'-Branes ist. Ebenso ist der Blowup (bzw. die Deformation) der verallgemeinerten Conifoldsingularität $\mathcal{G}_{k l} \quad T$-dual zu einem Intervall (einer Box) aus NS5/NS5'-Branes. Die beiden Singularitäten bilden ein Mirrorpaar im Sinne der lokalen Mirrorsymmetrie torischer Varietäten. Die Parameter der Kähler bzw. komplexen Struktur der Singularität entsprechen den Positionen der 
NS5-Branes im dualen Branebild. Die Verbindung zwischen der Box- und der Intervalltheorie wird durch das Zusammenziehen von 2-Zykeln und nachfolgende Öffnung von 3-Zykel der Mirrorpaare hergestellt ("conifold transition"). Die singulären Raumzeiten und die NS5-Branekonfigurationen repräsentieren die Hintergrundmetrik, die durch D-Branes ausgetestet wird.

Um den Zusammenhang zwischen Conifoldsingularitäten und Brane-Boxen herzustellen, muß das Konzept der Brane-Box durch Einschluß von Branediamanten erweitert werden. Im Ergebnis dessen lassen sich exakte Regeln zur Ableitung der auf der Brane-Box lebenden Eichtheorie und ihrer Materiefelder angeben, die die Regeln von [5] verallgemeinern. Ausgezeichnete singuläre Raumzeiten besitzen sowohl eine duale Beschreibung durch eine Brane-Box als auch durch ein Intervall. Die entsprechenden Eichtheorien auf der BraneBox und auf dem Intervall sind "mirror" zueinander. Das bedeutet folgendes: Während die Einbettung der Intervalltheorie in der M-Theorie durch einen supersymmetrischen 2-Zykel beschrieben wird, wird die Brane-Box durch einen supersymmetrischen 3-Zykel in die M-Theorie eingebettet. Diese Beobachtung sollte nützlich für die nichtstörungstheoretische Beschreibung dieser Klasse von $N=1$ Eichtheorien sein.

Im 3. Kapitel beginnen wir mit einer formalen Untersuchung der M5-Brane. Diese folgt der Idee aus dem letzten Kapitel, das eine Äquivalenz in der Beschreibung von Eichtheorien durch supersymmetrische 2-Zykel und supersymmetrische 3-Zykel postulierte. Im Zentrum steht das "Projektionsprinzip", das aus supersymmetrischen Zykeln supersymmetrische Zykel kleinerer Dimension konstruieren soll. Ein naives Projektionsprinzip funktioniert nicht, aber wir wollen durch eine konkrete Rechnungen zeigen, daß nach Einbeziehung der selbstdualen 3-Form $H$ eine Konstruktion in diese Richtung möglich wird. Die Grundlage für diese Analyse ist die intrinsische Formulierung der Weltvolumentheorie der M5-Brane, wie sie in [7] ausgearbeitet wurde. Das Hauptresultat dieses Kapitels ist die Ableitung von Differentialgleichungen, die die Struktur der M5-Brane nach dem Anschalten der selbstdualen 3-Form $H$, beschreiben. Im Fall des supersymmetrischen 2-Zykels ist es möglich, die 3-Form $H$ mit den Parametern der komplexen Struktur auf $\mathbb{R}^{4}$ zu identifizieren. Im Fall des supersymmetrischen 3-Zykels ist die Geschlossenheit von $H$ äquivalent zu einer deformierten Laplace-Gleichung.

In Kapitel 4 [9] wird die Diskussion von Brane-Boxen wieder aufgenommen. Das in den vorhergehenden Kapiteln aufgehäufte heuristische Material deutet darauf hin, daß die in einem 3-Zykel enthaltenen Informationen zumindestest teilweise aus geeignet konstruierten 2-Zykeln extrahiert werden kann. Aus diesem Grunde studieren wir Brane-Boxen, die explizit aus $N=2$ Untersystemen [10], bestehend aus D4 und NS5-Branes, aufgebaut sind. Diese Konstrukte erfüllen die Bedingung des 'uniform bending' [11] und der Lift auf einen supersymmetrischen 3-Zykel kann explizit durchgeführt werden. Die Struktur 
dieser 3-Zykel ist sehr speziell und besteht im wesentlichen aus einer Vereinigung der die $N=2$ Untersysteme beschreibenden 2-Zykel. Wir zeigen, daß die so konstruierten 3 -Zykel tatsächlich die richtige Information über die $\beta$-Funktion enthalten, indem wir den Koeffizienten $b_{0}$ extrahieren.

Die Untersuchung von D3-Branes in singulären Raumzeiten hängt eng mit der sogenannten AdS/CFT-Korrespondenz [12] zusammen. Diese gibt uns eine Möglichkeit zur dualen Beschreibung der $N=4$ Super-Yang-Mills Theorie (SYM), d.h. der Weltvolumentheorie von D3-Branes, durch IIB Supergravitation auf $A d S_{5} \times S^{5}$. Das Studium von Eichtheorien an Orbifoldsingularitäten führt zu einer Erweiterung dieser Korrespondenz. 6-dimensionale Orbifoldsingularitäten $\mathcal{O}=\mathbb{R}^{6} / \Gamma, \Gamma$ eine diskrete Gruppe, wurden in $[13,14]$ untersucht. Die Modifikation auf der Supergravitationsseite besteht in der Ersetzung von $S^{5}$ durch $S^{5} / \Gamma$. Die Orbifoldeichtheorie kann analog zum Beispiel im 1. Kapitel berechnet werden. Die Conifoldsingularität [15] führt ganz analog zu der Ersetzung von $S^{5}$ durch den homogenen Einsteinraum $T^{1,1}$.

In Kapitel 5 [17] werden neue superkonforme $N=1$ Eichtheorien konstruiert, die sich aus Deformationen der $N=4$ SYM ergeben und eine duale Supergravitationsbeschreibung durch IIB auf $A d S_{5} \times M^{5}$ besitzen. $M^{5}$ ist ein 5-dimensionaler Einsteinraum. Wir wollen zeigen, daß ein Massenterm für eines der drei chiralen Multipletts der $N=4$ SYM eine effektive Theorie der masselosen Felder zur Folge hat, die dual zu der $A d S_{5}$-Lösung mit $N=2$ Supersymmetrie aus [18] ist. In [18] konnte die duale Eichtheorie nicht identifiziert werden. 


\section{Selbständigkeitserklärung}

Ich versichere hiermit, die vorliegende Arbeit selbständig angefertigt zu haben und keine weiteren als die angegebenen Hilfsmittel verwendet zu haben. 


\section{Lebenslauf}

$\begin{array}{ll}\text { Name: } & \text { André Miemiec } \\ \text { Geburtsdatum: } & \text { 22. September } 1971 \\ \text { Geburtsort: } & \text { Berlin } \\ \text { Nationalität: } & \text { Deutsch }\end{array}$

Ausbildung:

seit 1997 Promotionsstudium an der Humboldt-Universität zu Berlin Mitglied des Graduiertenkollegs "Strukturuntersuchungen, Präzisionstests und Erweiterungen des Standardmodells" Thesis advisor: Prof. Dr. D. Lüst

1996 - 1997 Physikdiplom an der Humboldt-Universität Berlin

Thema: "Topologische Aspekte Magnetischer Monopole in Feld (String) Theorie"

Wiss. Betreuer: Prof. Dr. D. Lüst

1991 - 1996 Physikstudium an der Humboldt-Universität

seit 1995 Mitglied der "Studienstiftung des Deutschen Volkes"

1988 - 1990 "Max-Planck" - Gymnasium, Berlin 


\section{Publikationsliste}

\section{Veröffentlichungen}

A. Karch, D. Lüst, A. Miemiec $\mathbf{N}=\mathbf{1}$ Supersymmetric Gauge Theories and Supersymmetric 3-cycles Nucl.Phys. B553 (1999) 483-510, hep-th/9810254

A. Karch, D. Lüst, A. Miemiec New N=1 Superconformal Field Theories and their Supergravity Description Phys.Lett. B454 (1999) 265-269, hep-th/9901041

M. Aganagic, A. Karch, D. Lüst, A. Miemiec Mirror Symmetries for Brane Configurations and Branes at Singularities hep-th/9903093, erscheint in Nucl.Phys. B

D. Lüst, A. Miemiec Supersymmetric M5-branes with H-field hep-th/9912065, erscheint in Phys.Lett. B

\section{Beiträge zu Proceedings}

A. Karch, D. Lüst, A. Miemiec, Supersymmetric 3-Cycles for N=1 Brane Box Models, Proceedings der 2. Internationalen Konferenz auf Korfu, Griechenland, 20.-26. September 1998.

\section{Vorträge auf Konferenzen}

A. Karch, D. Lüst, A. Miemiec, Some Steps To The Calculation Of SUSY-ThreeCycles, gehalten auf dem 32. Internationalen Symposium Ahrenshoop zur Theorie der Elementarteilchen, Buckow, Deutschland, 1.-5. September 1998 